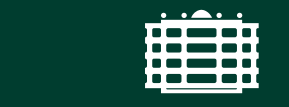

TECHNISCHE UNIVERSITÄT CHEMNITZ
Universitätsbibliothek

Europäische Union. Europäischer Fonds für regionale Entwicklung. Evropská unie. Evropský fond pro regionální rozvoj.

\title{
LERNRAUM - BIBLIOTHEKARISCHE INFORMATIONSPLATTFORM
}

Erfahrungen und Ergebnisse des Kooperationsprojektes von Bibliotheken in Sachsen und Tschechien 


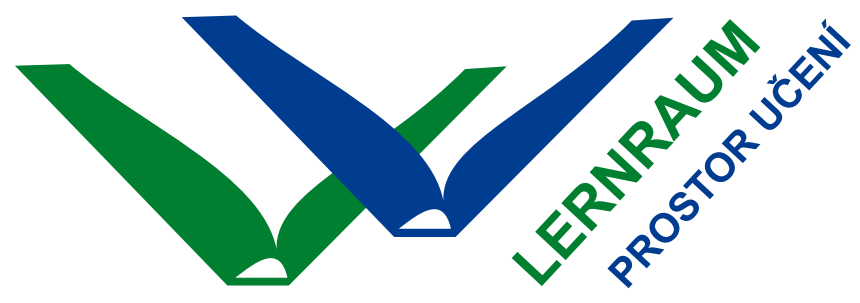

\section{LERNRAUM - BIBLIOTHEKARISCHE INFORMATIONSPLATTFORM}

Erfahrungen und Ergebnisse des Kooperationsprojektes von Bibliotheken in Sachsen und Tschechien

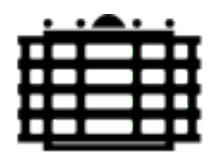

TECHNISCHE UNIVERSITÄT CHEMNITZ

Universitätsverlag Chemnitz 2018 


\section{Impressum}

\section{Bibliografische Information der Deutschen Nationalbibliothek}

Die Deutsche Nationalbibliothek verzeichnet diese Publikation in der Deutschen Nationalbibliografie; detaillierte bibliografische Angaben sind im Internet über http://www.dnb.de abrufbar.

\section{Bibliografische Information der Tschechischen Nationalbibliothek}

Die Tschechische Nationalbibliothek verzeichnet diese Publikation in der Tschechischen Nationalbibliografie; detaillierte bibliografische Angaben sind im Internet über https://www.nkp.cz abrufbar.

Satz / Layout:

Jakub Pokorný, Westböhmische Universität in Pilsen / Universitätsbibliothek Übersetzung:

Martina Pál, Helena Neumannová

Lektorat:

Veronika Jahn, Markéta Siegel, Cornelia Oertel

Titelbild:

Rico Welzel, Technische Universität Chemnitz

Technische Universität Chemnitz / Universitätsbibliothek

\section{Universitätsverlag Chemnitz}

09107 Chemnitz

https://www.tu-chemnitz.de/ub/univerlag

readbox unipress

in der readbox publishing $\mathrm{GmbH}$

Am Hawerkamp 31

48155 Münster

http://unipress.readbox.net

ISBN 978-3-96100-063-0

http://nbn-resolving.de/urn:nbn:de:bsz:ch1-qucosa2-314967

DOI 10.15240/tul/003/9783961000630

https://dspace.tul.cz/handle/15240/26601 
Diese Publikation entstand als Ergebnis des Projektes Lernraum - Bibliothekarische Informationsplattform, welches im Rahmen des Kooperationsprogrammes zur Förderung der grenzüberschreitenden Zusammenarbeit zwischen dem Freistaat Sachsen und der Tschechischen Republik 2014-2020 durch die Europäische Union gefördert wurde.

Folgende fünf Projektpartner nahmen an diesem Projekt teil:

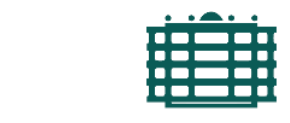

TECHNISCHE UNIVERSITÄT

CHEMNITZ
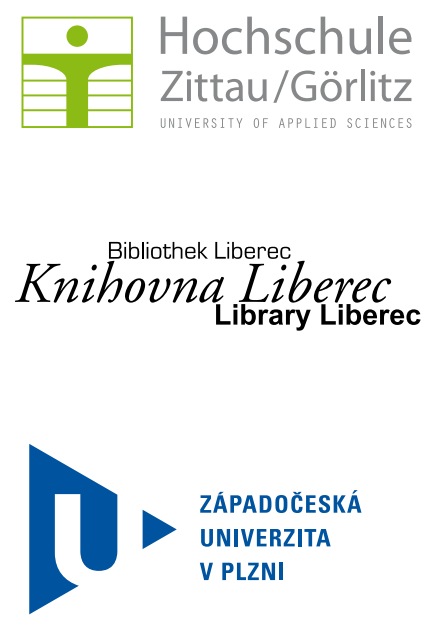

TECHNICKÁ UNIVERZITA V LIBERCI
Univerzitní knihovna

Weitere Informationen findet man unter:

https://www.bibliothek.tu-chemnitz.de/lernraum 


\section{Autoren:}

\section{Angela Malz}

Universitätsbibliothek der Technischen Universität Chemnitz

\section{Monika Ullmann}

Universitätsbibliothek der Technischen Universität Chemnitz

\section{Falk Maiwald}

Hochschulbibliothek der Hochschule Zittau/Görlitz

\section{Ralf Schwarzbach}

Hochschulbibliothek der Hochschule Zittau/Görlitz

\section{Miloslava Faitová}

Universitätsbibliothek der Westböhmischen Universität in Pilsen

\section{Magda Šrajbová}

Universitätsbibliothek der Westböhmischen Universität in Pilsen

\section{Zdeňka Firstová}

Universitätsbibliothek der Westböhmischen Universität in Pilsen

Miroslava Pourová

Universitätsbibliothek der Westböhmischen Universität in Pilsen

Blanka Konvalinková

Wissenschaftliche Bezirksbibliothek in Liberec

Dana Petrýdesová

Wissenschaftliche Bezirksbibliothek in Liberec

Jitka Vencláková

Universitätsbibliothek der Technischen Universität in Liberec

\section{Václav Ovčačík}

Universitätsbibliothek der Technischen Universität in Liberec 
INHALT 


\section{Falk Maiwald}

1 Lernräume ohne Grenzen ......................................................................... 7

Monika Ullmann / Angela Malz

2 Elektronische Medien und Fernleihe im grenzübergreifenden Konsortium

Falk Maiwald

3 Medientechnik und Infrastrukturen im Netzwerk-Lernraum

Zdeňka Firstová / Miroslava Pourová

4 OPEN ACCESS

Angela Malz / Václav Ovčačík

5 Informationskompetenz im grenzübergreifenden Lernraum

Dana Petrýdesová / Blanka Konvalinková

6 Erkenntnisse einer erfolgreichen Zusammenarbeit

Angela Malz / Falk Maiwald / Ralf Schwarzbach / Miloslava Faitová / Magda Šrajbová / Blanka Konvalinková / Dana Petrýdesová / Jitka Vencláková 


\section{LERNRÄUME OHNE GRENZEN}

Falk Maiwald 


\subsection{Partner und Ziele des Projektes}

Im Rahmen des Kooperationsprogrammes zur Förderung der grenzüberschreitenden Zusammenarbeit zwischen dem Freistaat Sachsen und der Tschechischen Republik 2014-2020 führten die Universitätsbibliothek Chemnitz, die Hochschulbibliothek Zittau/Görlitz, die Universitätsbibliothek in Pilsen, die Wissenschaftliche Bezirksbibliothek in Liberec und die Universitätsbibliothek in Liberec gemeinsam das Projekt Lernraum - Bibliothekarische Informationsplattform durch. Damit traten die fünf Projektpartner in ein sehr aktuelles Themengebiet ein - die Gestaltung von Bibliotheken als Lernräume. Anders als in der üblichen Diskussion im Bibliothekssektor stand dabei nicht der jeweils eigene Lernraum im Vordergrund. Das oberste Ziel des Projektes war vielmehr die Bildung eines gemeinsamen, nachhaltigen grenzüberschreitenden Lernraums. Alle Projektanstrengungen sollten auf dieses Ziel ausgerichtet sein. Um dieses komplexe Vorhaben aber sukzessive und erfolgreich bewältigen zu können, war

- die Festlegung klar abgegrenzter Meilensteine (siehe Kapitel 1.4),

- ein abgestimmtes, strukturiertes Vorgehen,

- ein gemeinsames Verständnis und

- die Akzeptanz der Schritte und Maßnahmen in den Projektmeilensteinen

von allen Projektpartnern erforderlich. Die Basis dafür schafften grundsätzliche Überlegungen zur Relevanz und zu allgemeinen und projektspezifischen Inhalten des Themas Lernraum (siehe Kapitel 1.3) sowie zu den einzelnen Meilensteinen (siehe Kapitel 1.4). Wie bei allen Projekten hing der Erfolg von den Akteuren und deren Kooperationsbereitschaft ab. Darüber hinaus war zu klären, welcher Nutzen bzw. welche Effekte durch das Projekt erzielt werden sollen (siehe Kapitel 1.2).

\subsection{Kooperation als Basis eines grenzüberschreitenden Bibliotheks-Lernraums}

Regionale bis hin zu nationalen Kooperationen von Bibliotheken sind sowohl innerhalb von Tschechien als auch in Sachsen bzw. Deutschland seit langem in verschiedenen Themenbereichen etabliert. Gründe der Zusammenarbeit von wissenschaftlichen Bibliotheken liegen beispielsweise im gegenseitigen Aufbau und im Austausch von Wissen und Erfahrungen und in der Ausnutzung 
von Effekten durch Ressourcenverbünde (z.B. beim Erwerb von E-Medien, Fernleihe von Büchern, Semesterapparate). Diese Gründe können auch für internationale Bibliotheks-Kooperationen angeführt werden, wenngleich hier sowohl inhaltliche, kulturelle, politische und rechtliche Unterschiede eine besondere Rolle spielen können.

In Grenzräumen bieten sich grenzüberschreitende Kooperationen aufgrund der geografischen Nähe der potenziellen Kooperationspartner an. Ausgangspunkt ist, dass Nutzer in ihrem regionalen Einzugsbereich jenseits der Grenze für sich relevante wissenschaftliche Bibliotheken vorfinden, wie beispielsweise in Zittau respektive Liberec. Insofern verwundert es nicht, dass bereits seit vielen Jahren enge Kontakte und eine lebendige Kooperation zwischen der Hochschulbibliothek Zittau/Görlitz auf der einen sowie der Wissenschaftlichen Bezirksbibliothek in Liberec und der Universitätsbibliothek in Liberec auf der anderen Seite bestehen. ' Eine grenzüberschreitende Bibliothekskooperation kann zudem auch durch die Kooperation der zugehörigen Hochschulen bzw. Universitäten begründet sein, wie beispielsweise in Gestalt der Semesterapparate der Universitätsbibliothek Chemnitz und der Universitätsbibliothek der Westböhmischen Universität in Pilsen. ${ }^{2}$

Diese langfristig ausgerichteten bi- und trilateralen Bündnisse werden in dieser oder ähnlicher Form auch weiterhin Bestand haben. Denn der Wille des grenzüberschreitenden gemeinsamen Gestaltens, der gegenseitige Respekt und das freundschaftliche Vertrauen stärken diese Verbundenheit und werden erhalten bleiben. Allerdings ergaben und ergeben sich auch Ansätze, bei denen eine temporäre Kooperation mit weiteren Partnern, die ähnliche Problem- und Fragestellungen zu lösen bzw. zu beantworten haben, in Form von Projekten sinnvoll ist.

Bereits 2009 startete die Universitätsbibliothek Chemnitz ein Projekt mit der Universität Jan Evangelista Purkyně (UJEP) in Ústí nad Labem und der Universitätsbibliothek der Westböhmischen Universität in Pilsen. ${ }^{3}$ Bei dieser erstmaligen Projektkooperation zwischen den Nachbarland-Bibliotheken lag der Schwerpunkt im Ausbau der Sächsisch-Tschechischen Fachbibliothek und im grenzüberschreitenden Leihverkehr. Das Projekt Sächsisch-Tschechisches

\footnotetext{
1 Vgl. Maiwald (2016).

2 Vgl. Slabý (2014).

${ }^{3}$ Vgl. Malz (2010).
} 
Bibliotheksnetzwerk - Informationen grenzenlos folgte von 2013-2014. Projektpartner waren die Universitätsbibliothek der Technischen Universität Chemnitz, die Hochschulbibliothek Zittau/Görlitz, die Universitätsbibliothek der Westböhmischen Universität in Pilsen und die Wissenschaftliche Bezirksbibliothek in Liberec. Ziel des Projektes war der Aufbau eines nachhaltigen und effizienten Bibliotheksnetzwerkes zwischen Sachsen und Tschechien.

Mit dem in dieser Arbeit reflektierten Projekt Lernraum - Bibliothekarische Informationsplattform intensivierte sich die Zusammenarbeit weiter - diesmal unter Einbeziehung der Universitätsbibliothek der Technischen Universität in Liberec und mit sehr ambitionierten Zielen und Aufgaben im Kontext "Lernraum”.

\section{Nutzen der Kooperation und des Projektes}

Gleich zu Beginn des Projektes, im Rahmen der Auftaktveranstaltung im Januar 2017, diskutierten die Projektpartner den erwarteten Nutzen bzw. die Chancen des Projektes. Dabei wurde in intern und extern wirkende Effekte unterschieden.

Für die kooperierenden Bibliotheken lagen die wichtigsten auf sie wirkenden internen Effekte in

- der direkten territorialen Nähe,

- den ähnlichen Themen- und Problemstellungen und entsprechenden Lösungen,

- dem voneinander Lernen - miteinander Lernen - übereinander Lernen,

- der hochschulinternen Stärkung der "Marke Bibliothek" (Leistungsfähigkeit, up to date),

- der Stärkung der Beziehungen zwischen den Hochschulen - Bibliothek als Lernraum-Vorreiter,

- dem Abbau von „Barrieren“ und einem niederschwelligen Zugang zu den anderen Bibliotheken.

Als externe, das heißt im gesamten Grenzraum und darüber hinaus wirkende Effekte, erhofften sich die Projektpartner

- die Erweiterung des Angebotsspektrums im kooperativen Zusammenschluss („Brücken bauen”) durch moderne Infrastrukturen, gemeinsam nutzbare Medien (E-Books und Fernleihe),

- die Verbesserung der Informationskompetenz der Nutzer, 
- die Erhöhung der Nutzerzufriedenheit,

- damit einen Einfluss auf die positive Positionierung der eigenen Hochschule bei potentiellen Studierenden und bei Multiplikatoren (Eltern, Lehrer),

- die Stärkung der Wissenschaftslandschaft in der Region,

- die Stärkung des Open Access-Gedankens,

- die Stärkung der Wahrnehmung und der Reputation der "Marke Bibliothek",

- einen Referenzcharakter, das heißt das Bibliotheksnetzwerk als Vorreiter für grenzüberschreitende Kooperationen und Lernräume anderer Bibliotheken.

\subsection{Lernräume wissenschaftlicher Bibliotheken und Lernraum des Projektes}

„Lernräume verbinden die Gesamtheit aller Maßnahmen, Ressourcen, Infrastruktur und Services zur Gestaltung der Lern- und Arbeitssituation der Studierenden"4, die diese "zum Zweck des Lernens zeitlich begrenzt aufsuchen" ${ }^{\prime 5}$. Wissenschaftliche Bibliotheken haben in den letzten Jahren eine starke Aufwertung als Lernräume erhalten. Auf der einen Seite wird diese Entwicklung getrieben durch veränderte Lernformen wie Projekt- und Gruppenarbeiten, Blended Learning und die konzentrierte Verfügbarkeit von Print- und elektronischen Medien an einem Ort. Auf der anderen Seite haben sich auch die Bedürfnisse und Ansprüche der Lernenden im digitalen Zeitalter stark verändert.

Entsprechend verhalten sich die wissenschaftlichen Bibliotheken und richten die Gestaltung ihrer Lernräume an den Anforderungen ihrer Nutzer aus. Diverse, maßgeschneiderte Konzepte entstehen ${ }^{6}$, um das "neue" Lernen zu unterstützen. Dabei spielen digitale Technologien und Medien eine sehr wichtige Rolle. Die traditionelle Bibliothek, die ein Ort der Bücher war, hat sich zu einem Ort für diverse interne und externe Nutzergruppen gewandelt, die in der Bibliothek arbeiten, kommunizieren, neue Ideen entwickeln oder sich einfach nur treffen wollen.

\footnotetext{
${ }^{4}$ Vgl. DINI (2013), S. 7.

${ }^{5} \mathrm{Vgl}$. May/Kannenberg (2014), S. 9.

${ }^{6}$ Vgl. stellvertretend Kohl-Frey (2014), Rautenberg et al. (2014).
} 


\section{Systematisierung von Nutzergruppen wissenschaftlicher Bibliotheken}

Zu den internen Nutzern (siehe Abbildung 1) zählen natürlich in erster Linie die Studierenden. Aber auch Forschende und Lehrende arbeiten und lernen im Lernraum Bibliothek. Da es der Zweck von Lernräumen ist, durch die Gestaltung der Lernumgebungen Lernprozesse gewollt zu unterstützen und zu fördern7, betrifft dies auch die „lernende Organisation"8 Bibliothek selbst. Insofern sind in einem weiter gefassten Verständnis auch Lernprozesse der Mitarbeiterinnen und Mitarbeiter der Bibliotheken zu berücksichtigen, die den Lernraum zur Weiterbildung und zum fachlichen Austausch nutzen. Dieses Verständnis ist wegen seiner praktischen Relevanz bei Kooperationen (vgl. Kapitel 1.2) für dieses Projekt sehr wichtig.

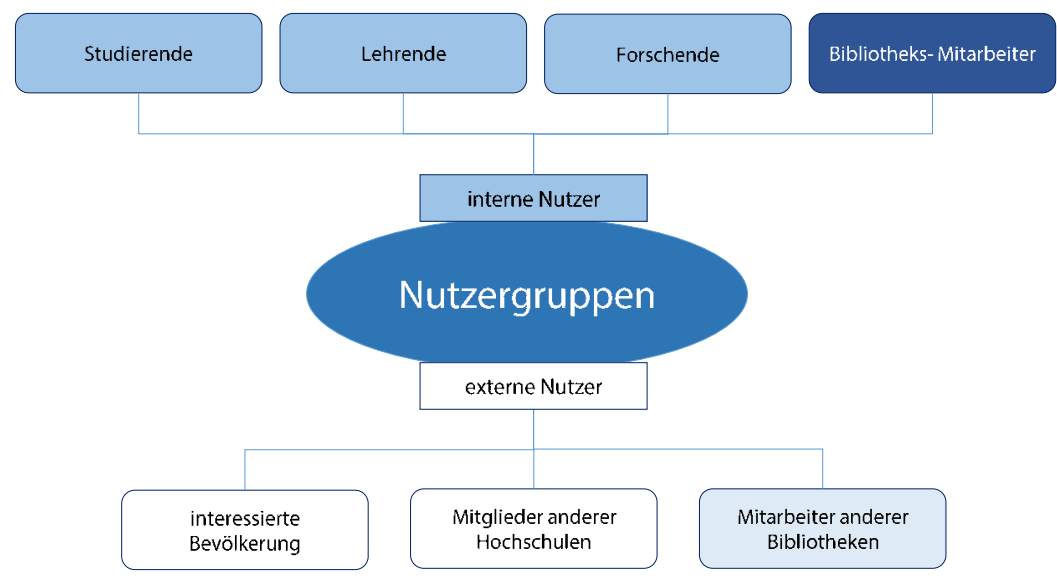

Abbildung 1. Systematik der Nutzergruppen im Lernraum.

Eine wissenschaftliche Bibliothek. Quelle: Eigene Darstellung.

Zu den externen Nutzern können im engeren Sinn Interessierte aus der Bevölkerung sowie Mitglieder anderer Hochschulen, also Studierende, Lehrende und Forschende, gezählt werden. Im weiteren Sinn gehören aber auch hier Mitarbeiter anderer Bibliotheken dazu.

7 Vgl. May/Kannenberg, 2014, S. 10.

8 Nach Senge (2011). 


\section{Prinzipielle Arten von Lernräumen}

Betrachtet man Lernräume aus verschiedenen Perspektiven, so können sie nach Wittwer/Diettrich (2015) in materiell-physische, soziale, virtuelle und Kooperations-Räume eingeteilt werden. ${ }^{9}$ Aus sozialer Sicht sind Lernräume Orte der Begegnung und damit sozialer Interaktion. Darüber hinaus geben sie aber auch Erwartungshaltungen vor, die die Nutzer mit dem Geschehen an diesem Ort verbinden. ${ }^{10}$

Im materiell-physischen Kontext sind Lernräume reale, in sich abgeschlossene physische Einheiten - im Regelfall innerhalb von Gebäuden -, die mit Infrastrukturen ausgestattet sind, die dem Nutzungszweck entsprechen. Dazu zählen Möbel, Technik und weitere raumgestalterische Elemente. ${ }^{11} \mathrm{Im}$ Folgenden werden die materiell-physischen Lernräume mit Berücksichtigung der sozialen Komponenten als lokale Lernräume bezeichnet.

Virtuelle Lernräume sollen hier als nicht-physische Lernräume verstanden sein, bei denen die Einheit von Raum und Zeit aufgehoben ist und die Kommunikation über Medien stattfindet. Sie benötigen als Ausgangspunkte zwar materiellphysische bzw. lokale Lernräume, sind aber an keine physischen Raumgrenzen gebunden. Mit Hilfe der Telekommunikation und der Internet-Technologien können sie sogar „weltumspannend” sein, d.h. geografische und (regelmäßig) politische Grenzen überschreiten. In diesem Kontext werden sie in dieser Arbeit als virtuelle standortübergreifende Lernräume bezeichnet. Ebenso wie lokale Lernräume haben sie soziale bzw. interkulturelle Dimensionen mit eigenen Wertemustern und Verhaltensregeln. ${ }^{12}$

Kooperationsräume dienen - typischerweise in Netzwerken oder (Modell-) Projekten - durch unterschiedliche Akteure und deren Interaktion und gemeinsame Arbeit zur gemeinsamen Problemlösung. Kooperationen (siehe Kapitel 1.2) entstehen, wachsen und entwickeln sich im Kooperationsprozess weiter, was zu einem anderen Agieren, Arbeiten und Lernen als in anderen sozialen Räumen führt. ${ }^{13}$

\footnotetext{
9 Vgl. Wittwer/Diettrich (2015), S. $15 \mathrm{ff}$.

10 Vgl. May/Kannenberg (2014), S. 10.

11 Vgl. May/Kannenberg (2014), S. 10.

12 Vgl. Wittwer/Diettrich (2015), S. 19, S. 21.

${ }^{13}$ Vgl. Wittwer/Diettrich (2015), S. 21.
} 


\section{Abgrenzung und Innovation des Lernraums im Projekt}

Für das Projekt wurden praktisch alle Perspektiven auf Lernräume verbunden. Ausgerichtet am obersten Ziel bestand die Aufgabe, einen grenzüberschreitenden Kooperations- bzw. Netzwerk-Lernraum zwischen den tschechischen und sächsischen Projektpartnern zu beschreiben und zu gestalten. Im Unterschied zur typischen Sichtweise (siehe Abbildung 1) mussten die potenziell interagierenden Nutzergruppen aller kooperierenden Projektpartner (Abbildung 2) einbezogen, gemeinsame Lerninteressen innerhalb von Sub-Lernräumen identifiziert sowie inhaltliche Themen und technische Lösungen für die lokalen und den standortübergreifenden Lernraum entwickelt werden. Damit sollte eine Bibliothekarische Informationsplattform entstehen.

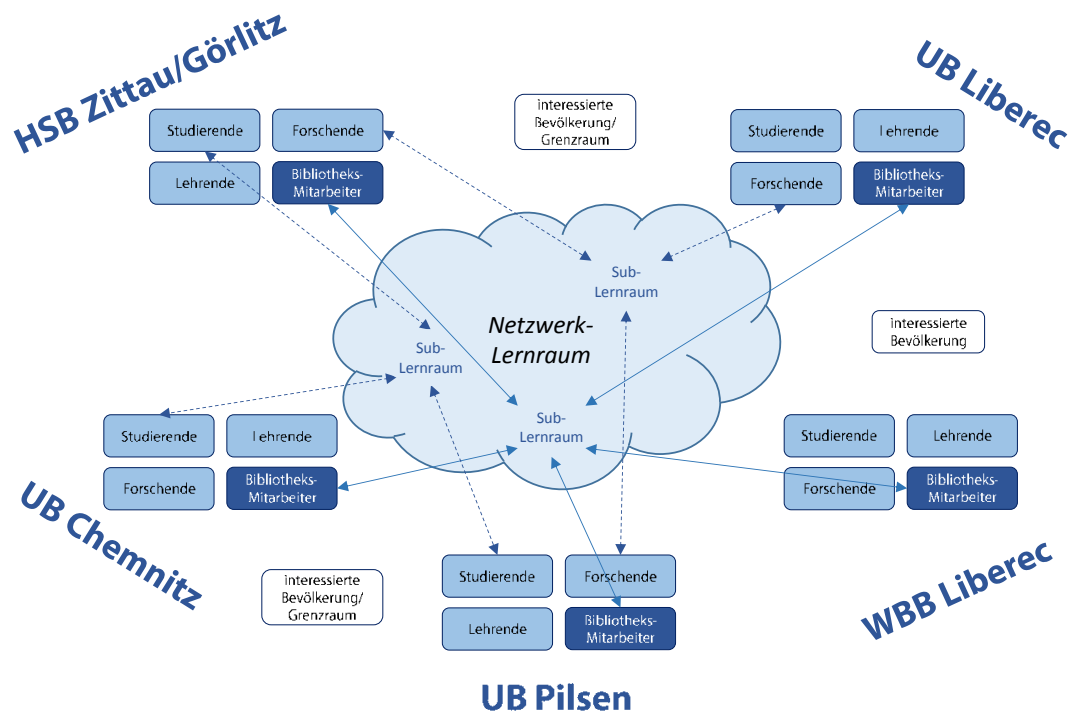

Abbildung 2. Schema des Netzwerk-Lernraums mit Sub-Lernräumen. Quelle: Eigene Darstellung.

Unter dem standortübergreifenden Netzwerk-Lernraum ist ein informeller Zusammenschluss von Lernenden, Forschenden, Lehrenden und/oder Bibliotheksmitarbeitern, die aufgrund gemeinsamer Interessen oder Problemstellungen kooperieren, Wissen und Erfahrungen austauschen und dabei voneinander lernen, zu verstehen. ${ }^{14}$ Mit dieser Definition erweiterte sich

${ }^{14}$ Angelehnt an Kupfer (2015), S. 204. 
das Blickfeld des Projektes im Vergleich zu vorangegangenen Projekten (siehe Kapitel 1.2) erheblich, denn nun spielten nicht mehr "nur" die reine Bibliotheksarbeit, sondern auch Beziehungen und Kooperationen zwischen anderen Akteuren der Hochschulen und Universitäten eine wichtige Rolle.

Ein materiell-physischer standortübergreifender Lernraum kann nur über Treffen am selben Ort zur selben Zeit, beispielsweise zu Beratungen, Fachkonferenzen, Workshops etc. realisiert werden. Im Projekt wurde dies vielfach in Form diverser Präsenz-Aktivitäten mit themenabhängig unterschiedlichen beteiligten Nutzergruppen praktiziert. Während an Workshops zu den Meilensteinen und Praxiswochen Projekt- und Bibliotheksmitarbeiter teilnahmen, waren die Veranstaltungen zur Steigerung der Informationskompetenz für die Studierenden, Lehrenden und Forschenden der Partner bestimmt. Für diese Zusammenkünfte stellte der jeweils ausrichtende Projektpartner seinen lokalen Lernraum zur Verfügung.

Das Innovative im Projekt war jedoch die Gestaltung eines virtuellen standortübergreifenden Lernraums, den es nach Kenntnisstand der Projektpartner zwischen Bibliotheken in Sachsen und Tschechien und auch darüber hinaus bislang noch nicht gab. Ein solcher virtueller Raum besteht prinzipiell, wenn interessierte Nutzergruppen über das Internet kommunizieren oder kollaborieren und so Orte und ggf. auch Zeit entkoppeln. Im Projekt-Lernraum wurden Webkonferenzen als typische Formen der Kommunikation zwischen verschiedenen Orten zur selben Zeit eingesetzt, insbesondere zur Projektabstimmung zwischen den Bibliotheken, aber auch für Kollaborationsszenarien der anderen Nutzergruppen. Für eine zeitversetzte grenzüberschreitende Kommunikation bzw. Kollaboration wurden E-Learning-Systeme und Web-Plattformen (siehe Kapitel 5), E-Mails sowie zentrale Datenspeicher zur Dateiablage eingesetzt. Entsprechende technische Voraussetzungen in den lokalen Lernräumen der Partner wurden genutzt bzw. im Rahmen des Meilensteins 3 geschaffen (siehe Kapitel 3).

\subsection{Ableitung projektrelevanter Inhalte im grenzübergreifenden Bibliotheks-Lernraum}

Die hohen Erwartungen an das Projekt (siehe Kapitel 1.2) resultierten aus den bereits im Projektantrag fixierten Projekt-Meilensteinen. 
Zu den Meilensteinen zählten:

1. Konsortiale Beschaffung von Elektronischen Medien; Fernleihe und Semesterapparate

2. Technische Ausstattung des Lernraumes

3. Open Access

4. Fachgerechte Vermittlung von Informationskompetenz

5. Veranstaltungen und Publizitätsmaßnahmen

Das am höchsten gesteckte Ziel im Meilenstein 1 war die erstmalige Realisierung eines tschechisch-deutschen Erwerbungskonsortiums für E-Books, die dann im Lernraum grenzübergreifend für alle Nutzer verfügbar sein sollten. Federführend bei der Anbahnung und den Verlags-Verhandlungen war der Leadpartner, die Universitätsbibliothek Chemnitz. Ein weiteres wichtiges Ziel war der Ausbau der grenzüberschreitenden Fernleihe, verbunden mit der Analyse nach Bedarfen und Lösungen für Semesterapparate (siehe Kapitel 2).

Im Meilenstein 2 galt es für die verantwortliche Hochschulbibliothek Zittau/ Görlitz, den innovativen Kern des virtuellen grenzüberschreitenden Lernraums durch die Analyse von Nutzungsszenarien zwischen den Nutzergruppen der Bibliotheken einzuleiten. Mit der daraus abgeleiteten Planung, Beschaffung und Implementierung entsprechender Computer- und Medientechnik, wie interaktive Whiteboards und Konferenztechnik, war der virtuelle Lernraum erfolgreich zu etablieren (siehe Kapitel 3).

Die Förderung des Open Access-Gedankens, das heißt des unbeschränkten und kostenlosen Zugangs zu wissenschaftlichen Informationen, und der Ausbau der Repositorien-Vernetzung zwischen den Partnern im Meilenstein 3 nahm sich die Universitätsbibliothek der Westböhmischen Universität in Pilsen verantwortlich an. Ein wichtiges Ziel war hier ein gemeinsamer Austausch zu den Fortschritten der Open Access-Aktivitäten der beteiligten Hochschulbibliotheken und die weitere Sensibilisierung potenzieller Autorinnen und Autoren (siehe Kapitel 4).

Die Steigerung der Informationskompetenz der Nutzer, also der Fähigkeit, Informationen zu finden, aufzubereiten und zu bewerten, ist ein Dauerthema für alle Bibliotheken. Im Meilenstein 4, den die WBB und die UB Liberec verantworteten, war eine bessere Integration der Informationskompetenz in Lehre, Forschung und Studium anzustreben. Ein eigens entwickeltes, 
zweisprachiges Lernmodul zur Vermittlung von Informationskompetenz im lokalen und grenzüberschreitenden Lernraum - das heißt mit Bezug auf die Leistungen aller Projektpartner - soll dazu dienen. Weitere Konzepte waren im Rahmen des Projektes zu erörtern (siehe Kapitel 5).

Im Zentrum des 5. Meilensteins stand die hier nun vorliegende Publikation, die die Projektarbeit, die Projektergebnisse und die entstandenen Mehrwerte dokumentiert. Bestenfalls dient sie - zumindest teilweise - als Wegweiser für ähnliche Vorhaben anderer Bibliotheken.

\section{Literatur}

DINI, Deutsche Initiative für Netzwerkinformation e.V.: Die Hochschule zum Lernraum entwickeln, Kassel 2013.

May, A.; Kannenberg, S.: Entgrenzung und Zusammenarbeit - die Notwendigkeit von Kooperationen im Lernraum; in: ABI Technik, Heft 34 (1) 2014, S. 9-19.

Kohl-Frey, 0.: Die Universitätsbibliothek als neuer Lernraum: Konzept der Universität Konstanz; in: Eigenbrodt, 0.; Stang, R. (Hrsg.): Formierungen von Wissensräumen. Optionen des Zugangs zu Information und Bildung; Berlin/ Boston 2014, S. 107-123.

Kupfer, F.: Lernen und Arbeiten in Lernort übergreifenden Netzwerken, in: Wittwer, W.; Diettrich, A.; Walber, M. (Hrsg.): Lernräume, Wiesbaden 2015, S. 204-217.

Maiwald, F.: Internationale Kooperationen im Dreiländereck; in: BIS - Das Magazin der Bibliotheken in Sachsen, Dresden: Sächsische Landesbibliothek - Staats- und Universitätsbibliothek Dresden, Nr. 3/2016, S. 168-169.

Malz, A.: Grenzüberschreitende Kooperation: Die Universitätsbibliotheken in Chemnitz, Plzeň und Ústí nad Labem arbeiten zusammen; in: BIS - Das Magazin der Bibliotheken in Sachsen, Dresden: Sächsische Landesbibliothek - Staats- und Universitätsbibliothek Dresden, Nr. 4/2010, S. 254-255.

Rautenberg, K.; Klein, A.; Usinger, J.; Dombrowe, A.: Vom Lesesaal zum Learning Center - Ein neues Raum- und Benutzungskonzept an der UB Mannheim; in: b.i.t. online, Nr. 4/2014, S. 321-328.

Senge, P: Die fünfte Disziplin. Kunst und Praxis der lernenden Organisation. Klett-Cotta, 11. Aufl. 2011. 
Slabý, Adam: Damit einschlägige Werke verfügbar werden, 2014, unter: https://www.tu-chemnitz.de/tu/pressestelle/aktuell/5960, abgerufen am 22.08.2018.

Wittwer, W.; Diettrich, A.: Zur Komplexität des Raumbegriffs; in: Wittwer, W.; Diettrich, A.; Walber, M. (Hrsg.): Lernräume. Gestaltung von Lernumgebungen für Weiterbildung; Wiesbaden 2015, S. 1127. 


\section{ELEKTRONISCHE MEDIEN UND FERNLEIHE IM GRENZÜBERGREIFENDEN KONSORTIUM}

Monika Ullmann

Angela Malz 


\subsection{Die Sächsisch-Tschechische Fachbibliothek als Vorläuferin des grenzübergreifenden Konsortiums}

Lange vor dem Projekt Lernraum - Bibliothekarische Informationsplattform begannen sächsisch-tschechische Projekte an der Technischen Universität Chemnitz. Sie waren eng mit dem leider viel zu früh verstorbenen Herrn Prof. Dr. Peter Jurczek verbunden, der diese Zusammenarbeit initiiert hat. Von Beginn an wurden Mittel für den Aufbau einer Sächsisch-Tschechischen Fachbibliothek zur Verfügung gestellt.

Am Anfang stand im Jahr 2003 das Sächsisch-Tschechische Hochschulzentrum (STHZ). Ziel dieses Projektes war der Aufbau einer langfristig angelegten wissenschaftlichen Kooperation von Hochschulen im sächsisch-tschechischen Grenzgebiet. Es wurde mit Mitteln der EU-Gemeinschaftsinitiative Interreg III A kofinanziert.

Innerhalb dieses Projektes bekam die Universitätsbibliothek Chemnitz Mittel zugewiesen, um eine Sächsisch-Tschechische Fachbibliothek (STFB) aufzubauen. Mit den Beständen sollte die grenzüberschreitende wissenschaftliche Zusammenarbeit zwischen Tschechien und Sachsen gefördert werden. Die Universitätsbibliothek Chemnitz beschaffte Bücher in deutscher und tschechischer Sprache, die die deutsch-tschechische Zusammenarbeit zum Inhalt hatten. Fachbücher zu Wirtschaftsthemen, zur Geschichte von Sachsen und Böhmen oder zur EU-Osterweiterung kamen dazu. Es wurden auch belletristische Werke, Sprach- und Tourismusführer, Kartenmaterial, DVDs und Hörbücher in den Bestand aufgenommen. ${ }^{15}$ Inzwischen ist diese Fachbibliothek auf 11123 Medien angewachsen.

2006 lief das Projekt STHZ aus. Im Nachfolgeprojekt Sächsisch-Tschechisches Hochschulkolleg, das auch aus EU-Mitteln kofinanziert wurde, standen wieder Mittel für die Fachbibliothek zur Verfügung.

Im Sommer 2009 begann die Sächsisch-Tschechische Hochschulinitiative. Kofinanziert wurde das Projekt durch das Ziel 3-Programm des Europäischen Fonds für regionale Entwicklung sowie aus Mitteln des Freistaates Sachsen, der Tschechischen Republik und der beteiligten Universitäten.

Erstmals spielten die Bibliotheken der teilnehmenden Universitäten im Projektantrag eine größere Rolle. Betont wurden neben dem Ausbau der

${ }^{15}$ https://www.tu-chemnitz.de/ub/STHZ/sthz_medien.html 
Sächsisch-Tschechischen Fachbibliothek der grenzüberschreitende Leihverkehr und die verstärkte Kooperation der Bibliotheken.

Die Partner der Universitätsbibliothek Chemnitz waren damals die Bibliothek der Universität Jan Evangelista Purkyně (UJEP) in Ústí nad Labem und die Universitätsbibliothek der Westböhmischen Universität in Pilsen.

Am Ende des Projektes stand fest, dass die Bibliotheken weiter zusammenarbeiten wollen und andere Bibliotheken des sächsisch-tschechischen Grenzraumes einbeziehen möchten. So entstand das Projekt SächsischTschechisches Bibliotheksnetzwerk - Informationen grenzenlos, welches vom 01.12.2013 bis 30.11.2014 durchgeführt wurde. Projektpartner waren die Universitätsbibliothek der Technischen Universität Chemnitz, die Hochschulbibliothek Zittau/Görlitz, die Universitätsbibliothek der Westböhmischen Universität in Pilsen und die Wissenschaftliche Bezirksbibliothek in Liberec. Ziel des Projektes war der Aufbau eines nachhaltigen und effizienten Bibliotheksnetzwerkes zwischen Sachsen und Tschechien.

Die in diesem Projekt gesammelten positiven Erfahrungen und die persönlichen Kontakte führten im Jahr 2015 zu dem Entschluss, innerhalb des Kooperationsprogramms zur Förderung der grenzübergreifenden Zusammenarbeit zwischen dem Freistaat Sachsen und der Tschechischen Republik 2014-2020 in einem neuen Projekt Lernraum - Bibliothekarische Informationsplattform zusammenzuarbeiten. Projektpartner waren die Universitätsbibliothek der Technischen Universität Chemnitz, die Hochschulbibliothek Zittau/ Görlitz, die Universitätsbibliothek der Westböhmischen Universität in Pilsen, die Universitätsbibliothek der Technischen Universität in Liberec und die Wissenschaftliche Bezirksbibliothek in Liberec.

\subsection{Der Meilenstein 1 im Projekt Lernraum - Bibliothekarische Informationsplattform}

In diesem Projekt sollte endlich das geschafft werden, was in den vorhergehenden Projekten zwar immer diskutiert, aber aufgrund der kurzen Projektlaufzeiten nicht realisierbar war - das erste sächsisch-tschechische Konsortium für den Erwerb elektronischer Bücher. In einem Konsortium schließen sich Bibliotheken zusammen und bilden eine Einkaufsgemeinschaft mit dem Ziel, bessere Konditionen zu bekommen als im Alleinerwerb. Die 
Besonderheit im Lernraum-Projekt sollte die grenzübergreifende Nutzung der Medien sein, d.h. sie stehen den Nutzern aller Projektbibliotheken beiderseits der Grenze zu gleichen Bedingungen zur Verfügung.

Für die gedruckten Bücher, die in der Sächsisch-Tschechischen Fachbibliothek in der Universitätsbibliothek Chemnitz stehen und darüber hinaus für den gesamten Bücherbestand besteht die Möglichkeit, sie über die grenzüberschreitende Fernleihe an die Benutzer der tschechischen Bibliotheken auszuleihen. Außerdem stehen sie auch für Semesterapparate zur Verfügung.

Das Projekt Lernraum - Bibliothekarische Informationsplattform baute somit einerseits auf den Ergebnissen der vorhergehenden Projekte auf und verstetigt sie. Somit ist nach wie vor die Sächsisch-Tschechische Fachbibliothek mit den gedruckten Medien ein wichtiger Baustein der Zusammenarbeit. Andererseits greifen die Bibliotheken die Erfordernisse der digitalen Bibliothek auf und erwerben elektronische Medien.

Im Projektantrag steht dazu: „Der Lernraum baut auf vorhandene Wissensquellen auf. Die Bibliotheken bilden ein Konsortium und beschaffen elektronische Medien - eBooks. Diese Medien können grenzübergreifend von allen Bibliotheksbenutzern der beteiligten Bibliotheken genutzt werden. Ein Konsortium ermöglicht durch gemeinsamen Einkauf und größeres Volumen einen günstigeren Preis. Im Rahmen des Konsortiums werden auch diese Quellen Teil des Fernleihdienstes und der elektronischen Zusendung von Dokumenten sein. Die gedruckten Medien werden über den Weg der grenzüberschreitenden Fernleihe den Projektpartnern geschickt. Auf Wunsch und nach Bedarf der Lehre werden die Bücher auch für Semesterapparate zur Verfügung gestellt."

Die Aktivitäten zur Erreichung dieser Ziele waren:

- Workshop in der Hochschulbibliothek Zittau am 29.03.2017

- Praxiswoche in der Universitätsbibliothek Chemnitz vom 04. bis 07.12.2017

- Workshop in der Universitätsbibliothek in Pilsen am 14.02.2018

- Workshop in der Wissenschaftlichen Bezirksbibliothek in Liberec am 23.05.2018 
In Vorbereitung der Bildung des Sächsisch-Tschechischen Konsortiums zum Kauf elektronischer Medien wurden die Möglichkeiten der konsortialen Erwerbung in Tschechien und in Deutschland geprüft.

Elektronische Medien können in Tschechien größtenteils nur im Rahmen von Projekten, d.h. mit Fördermitteln angeschafft werden. Erwerbungsmodelle wie in Deutschland sind nicht realisierbar. Deshalb wurde entschieden, dass der Konsortialführer eine deutsche Bibliothek sein soll. Die Wahl fiel auf den Leadpartner, die Universitätsbibliothek Chemnitz.

\subsection{Konsortien in Deutschland}

In Deutschland gibt es verschiedene Modelle von Konsortien.

Für die Jahre 2004 bis 2010 wurde von der Deutschen Forschungsgemeinschaft (DFG) das Programm der Nationallizenzen ins Leben gerufen. Mit den Mitteln der DFG wurde der Erwerb abgeschlossener, elektronischer Ressourcen mit dauerhaften Archiv- und Hostingrechten gefördert: Volltextdatenbanken, Zeitschriftenarchive, E-Book-Sammlungen. Insgesamt wurden in diesem Programm für mehr als 100 Millionen EUR über 140 Nationallizenzen erworben. Die von der DFG bereitgestellten Fördermittel deckten alle Kosten ab. Zu den geförderten Produkten gehören u.a.:

- Early English Books Online

- NetLibrary

- The Making of the Modern World: economics, politics and industry

- Cambridge Journals Archives 1864-1996

- Springer Online Journals Archives 1860-2000

- Taylor \& Francis Online Archives 1799-2000

Teilnehmen konnten alle Benutzer wissenschaftlicher Bibliotheken sowie interessierte Privatnutzer in Deutschland. Die Teilnehmer mussten sich verpflichten, die ausgehandelten Nutzungsbedingungen zu akzeptieren. Verhandlungsführend war nicht die DFG, sondern die Bibliotheken aus dem Kreis der damaligen Sondersammelgebietsbibliotheken. Diese Bibliotheken übernahmen die Verhandlungen mit den einzelnen Verlagen, die Antragstellung, den Erwerb und den Abschluss der Lizenzverträge. Sie waren ebenfalls verantwortlich für die dauerhafte deutschlandweite Bereitstellung der Produkte. 
Verhandlungsführer waren:

- Bayerische Staatsbibliothek

- Deutsche Zentralbibliothek der Medizin

- Deutsche Zentralbibliothek der Wirtschaftswissenschaften

- Niedersächsische Staats- und Universitätsbibliothek Göttingen

- Staatsbibliothek zu Berlin - Preußischer Kulturbesitz

- Technische Informationsbibliothek

- Universitätsbibliothek Johann Christian Senckenberg

In den Jahren 2008-2010 wurde für die Lizenzierung fortlaufender Inhalte ein Pilotprojekt für laufende Zeitschriften getestet.

Durch die Bibliotheken, die auch schon verhandlungsführend für die Nationallizenzen waren, kam es zu zwölf Lizenzabschlüssen. Diese wurden nach zwei Modellen umgesetzt:

Modell 1 orientierte sich an den Nationallizenzen. Es wurden flächendeckende und vollfinanzierte Lizenzen abgeschlossen. Es handelte sich dabei aber ausdrücklich um Produkte der Spitzenversorgung. Die Vollfinanzierung erfolgte im Bereich der hochspezialisierten Ressourcen, die sich auch in einem finanziell begrenzbaren Bereich bewegten. Der Teilnehmerkreis entsprach den Teilnehmern der klassischen Nationallizenzen.

Zu den geförderten Produkten gehören:

- China Academic Journal

- Editoria Italiana Online Periodici

- Kluwer Law Journals

Im 2. Modell - einem „Opt-in-Modell“ mussten die Teilnehmer eine Eigenbeteiligung erbringen. Diese ließ keine flächendeckende Versorgung zu und auch der Zugriff durch Einzelnutzer war nicht mehr möglich. Es handelte sich damit um ein überregionales Konsortium mit DFG-Förderung.

Die Beibehaltung der zum Zeitpunkt des Vertragsbeginns vorhandenen Abonnements war der Anteil der teilnehmenden Einrichtungen. Die DFG übernahm den Aufpreis für den Zugriff auf das Gesamtangebot.

Einrichtungen ohne eigene Abonnements erhielten eine komplette Förderung. 
Bei den lizenzierten Paketen handelte es sich um Produkte kleinerer bzw. mittelgroßer Verlage:

- American Institut of Physics

- Annual Reviews

- British Medical Journal Publishing Group

- Institut of Physics Publishing

- Karger Publishers

- Oxford University Press

- Royal Society of Chemistry

- Sage Publications

- De Gruyter

Ergänzend zu den Nutzungsbedingungen der Nationallizenzen wurde das Konzept der Moving Wall eingeführt. Bei den von den zahlenden Teilnehmern lizenzierten Jahrgängen wurden diese mit ein oder zwei Jahren Verzögerung als flächendeckende Nationallizenz freigegeben.

Mit dem Ende der Förderung der Nationallizenzen und dem Abschluss des Pilotprojektes für laufende Zeitschriften ging die DFG-Förderung ab 2011 in die Schwerpunktinitiative "Digitale Information" der Allianz der Deutschen Wissenschaftsorganisation über. Ziel des Programms ist die nachhaltige Forschungsförderung und Sicherung der Konkurrenzfähigkeit des Wissenschaftsstandortes Deutschland.

Die Allianz-Initiative befasst sich neben der nationalen Lizenzierung auch mit nationaler Hostingstrategie, Forschungsdaten, Open Access, rechtlichen Rahmenbedingungen und virtuellen Forschungsumgebungen.

Dazu wurde die Arbeitsgruppe Allianz-AG Lizenzen gegründet, die sich aus Vertretern der an der Allianz-Initiative beteiligten Wissenschaftsorganisationen und durch die DFG berufene Vertreter von verhandlungsführenden Bibliotheken aus dem Nationallizenzen-Kontext zusammensetzt.

Das erprobte Opt-In-Modell wurde über die Zeitschriften hinaus für dynamische Ressourcen aller Art geöffnet. Die Förderquote durch die DFG beträgt dabei $\max .25 \%$.

Die Umstellung auf bestandsunabhängige Modelle erwies sich als schwierig. Benötigt wurden Preisparameter, die sich nicht am Abonnementstand orientieren, wie Nutzer (FTEs), Nutzung, Budget. 
Der Grundsatz des Erwerbs dauerhafter Verfügbarkeit gilt auch für die AllianzLizenzen. Dazu gehört auch die Moving Wall nach der jeweils ausgehandelten Frist.

Die Produktvorschläge der Bibliotheken werden von der Allianz-AG Lizenzen nach folgenden Kriterien überprüft:

- Kongruenz mit den Erwerbungsgrundsätzen für die DFG-geförderten Nationallizenzen

- Anzahl der Vorschläge/Dringlichkeit des Bedarfs

- Verfügbarkeit in Deutschland (mittlere Abdeckung, keine Nischen, keine Marktsättigung)

- Kosten-Nutzen-Aspekte

- Finanzierbarkeit

- Erfolgsaussichten der Verhandlungen

Bei den elektronischen Zeitschriften gehört auch eine Open AccessKomponente zu den vorgeschriebenen Bestandteilen einer Allianz-Lizenz. Ziel ist die Umsetzung des grünen Open Access, also die Möglichkeit, lizenzierte Artikel in institutsbezogene oder fachliche Repositorien zu übernehmen. Dies sollte möglichst in der vom Verlag veröffentlichten Form möglich sein. Für Institutionen und Autoren gibt es eine Produktübersicht zur Nutzung der verhandelten Open Access-Rechte.

Beispiele für die Allianz-Lizenzen sind:

- Brill Law Books online

- Cambridge Books Online

- MathSciNet - American Mathematical Society

- Nomos eBooks

Die Gasco (German, Austrian and Swiss Consortia Organisation) - Arbeitsgemeinschaft Deutscher, Österreichischer und Schweizer Konsortien - bündelt die regionalen Konsortialaktivitäten im deutschsprachigen Raum. Sie bildet eine Plattform zur strategischen Zusammenarbeit der Bibliotheken beim gemeinsamen Erwerb von elektronischen Zeitschriften, Datenbanken und E-Books.

In Deutschland gibt es regionale und institutionelle Konsortien. Die institutionellen Konsortien stammen aus dem Bereich der großen Forschungsverbünde (Fraunhofer-Gesellschaft, Leibniz Gemeinschaft, ...) und der Ressortforschung im Bereich der Bundesministerien. 
Die regionalen Konsortien bilden sich aus den Universitäts-, Hochschul- und Landesbibliotheken eines Bundeslandes. Diese können mit dem Einverständnis des Anbieters durch die Teilnahme von Hochschuleinrichtungen anderer Bundesländer erweitert werden. Bundesländer mit einer hohen Universitätsund Hochschuldichte, die meist auch überregional tätig sind, haben große und leistungsfähige Konsortien mit einer eigenen Geschäftsstelle. Geschäftsstellen mit eigenem Personal sind für die Bewältigung großer Konsortien wichtig und entlasten die Verhandlungsführer.

Die sächsischen wissenschaftlichen Bibliotheken bilden das Sachsenkonsortium. Die Verhandlungen erfolgen produktweise durch die angeschlossenen Universitäts- und Hochschulbibliotheken. Das Konsortium verfügt über keine eigene Geschäftsstelle.

\subsection{Sächsisch-Tschechisches Konsortium}

Für das im Projekt Lernraum - Bibliothekarische Informationsplattform zu bildende Konsortium mussten folgende Bedingungen eingehalten werden:

- Es mussten Verlage gefunden werden, die bereit sind, ein Angebot für ein grenzüberschreitendes Konsortium zur Verfügung zu stellen.

- Die Medien müssen dem fachlichen Profil aller beteiligten Projektpartner entsprechen.

- Es mussten aktuelle Titel nutzbar sein.

- Der Verlag musste eine internationale Ausrichtung haben.

- Es durfte keine Kollision mit dem sächsischen Konsortium geben.

Zur Auswahl standen die drei international agierenden Verlage Cambridge University Press, Sage Publications sowie die Verlagsgruppe Taylor \& Francis. Der erstgenannte deckt alle Fachgebiete der Interessenten ab, ist jedoch bereits im sächsischen Konsortium vertreten. Sage Publications spielt demgegenüber im sächsischen Konsortium keine Rolle, decken jedoch die gewünschten Themengebiete - dazu gehört besonders Technik - nicht ab und kamen damit nicht in Frage.

Taylor \& Francis mit den Imprints (ursprünglich eigene Verlage, die der Verlagsgruppe Taylor \& Francis angegliedert wurden) CRC, Routledge, Earthcan, Ashgate, Acumen, Pickering \& Chatto, Garland, Europa, Psychology Press und Spon deckt die Fächerprofile der teilnehmenden Projektpartner: Technik, 
Naturwissenschaften, Geisteswissenschaften, Wirtschaftswissenschaften, Sozialwissenschaften, Psychologie, Bauwesen und damit den Informationsbedarf der Projektpartner sehr gut ab. Taylor \& Francis ist nicht im sächsischen Konsortium vertreten. Die tschechischen Projektpartner hatten bereits aus Projektmitteln eine Zeitschriftendatenbank von Taylor \& Francis erworben.

Nach einem langen Auswahlprozess und vielen projektinternen Diskussionen entschieden sich die Projektpartner für das Angebot der Verlagsgruppe Taylor \& Francis, das über die Firma Missing Link vermittelt wurde. Dieser Schritt ermöglichte die Bildung des ersten sächsisch-tschechischen Konsortiums.

Für die Dauer der Projektlaufzeit verfügten alle Projektpartner über den Zugang zu einer umfangreichen Datenbank mit rund 5000 E-Books der Verlagsgruppe Taylor \& Francis im Wert von mehr als 670000 EUR. Nach Projektende werden aus dem Pool Bücher im Wert von 160000 EUR ausgewählt, die dann allen Projektbibliotheken dauerhaft zur Verfügung stehen. Diese Verfahrensweise wird in der Bibliothekswelt als Evidence-Based-Selection, kurz EBS bezeichnet.

Die eingesetzten finanziellen Mittel wurden effektiv angewendet und somit die Nachhaltigkeit des Projektes gesichert.

\subsection{Fernleihe und Semesterapparate}

Im sächsisch-tschechischen Lernraum spielt nach wie vor die Fernleihe von gedruckten Medien eine Rolle. Waren es in den ersten Projekten nur Medien aus der sächsisch-tschechischen Fachbibliothek, die über die Fernleihe ausgetauscht wurden, konnte dieser Service im Projekt Lernraum auf alle Medien der beteiligten Bibliotheken ausgedehnt werden. Zwischen den Projektpartnern wurde eine Vereinbarung geschlossen, in der folgende Regelungen festgelegt sind:

\section{Ausleihregeln}

\section{Bestand der Sächsisch-Tschechischen Fachbibliothek}

Die Leihfrist beträgt mindestens einen Monat, höchstens jedoch ein Jahr. Die Leihfrist für länger als einen Monat wird verkürzt, wenn das Medium durch einen anderen Interessenten angefordert wird. In diesem Fall ist das Medium sofort zurückzugeben. 
Nach Ablauf der maximalen einjährigen Frist kann das Medium bei Bedarf demselben Nutzer wieder ausgeliehen werden. Zu diesem Zweck wird es in der Heimatbibliothek des Ausleihenden vorgestellt. Nach der Bestätigung durch diese Bibliothek wird es in der Universitätsbibliothek Chemnitz erneut ausgeliehen.

\section{Bestand der Universitätsbibliothek der Technischen Universität Chemnitz (außer der Sächsisch-Tschechischen Fachbibliothek), der Hochschulbibliothek der Hochschule Zittau/Görlitz, der Universitätsbibliothek der Westböhmischen Universität in Pilsen, der Wissenschaftlichen Bezirksbibliothek in Liberec und der Universitätsbibliothek der Technischen Universität in Liberec}

Die Ausleihdauer beträgt einen Monat. Es ist möglich diese zweimal zu verlängern. Verlängerungen müssen beantragt werden. Weitere Regeln werden durch die ausleihende Bibliothek bestimmt.

\section{Kopien}

Kopien von Aufsätzen aus Zeitschriften, Buchkapiteln und Sammelbänden werden in Papierform bereitgestellt.

Mit diesen Regeln und dem Grundsatz der Direktbelieferung weichen die Bibliotheken von den nationalen Vorschriften in Deutschland und in Tschechien ab.

In Tschechien gilt das Prinzip der Regionalität und der Gegenseitigkeit. Die Bedingungen für die Fernleihe legt jede Bibliothek selbst fest. In das System der Fernleihe sind das Nationale Zentrum (Nationalbibliothek), die regionalen Zentren (Bezirksbibliotheken), die Bibliotheken mit Regionalfunktion und Spezialbibliotheken eingebunden. Die internationale Fernleihe ist bei 6 Bibliotheken möglich. Vor dem Auslösen einer Fernleihe kann man im tschechischen Gesamtkatalog recherchieren, der einen Bestand von 6850000 Titeln aufweist.

Seit 2016 läuft Das zentrale Portal der Bibliotheken (www.knihovny.cz), das die Suche in einem Gesamtkatalog der beteiligten Bibliotheken in einer Schnittstelle ermöglicht. Darüber hinaus entwickelt derzeit die Nationalbibliothek für Technik das Portal Získej (gewinne/erwerbe es!) - diese Plattform wird Fernleihe sowie die Dokumentenlieferung sicherstellen.

In Deutschland regelt die Leihverkehrsordnung die Fernleihe. Auch hier gibt es das Regionalprinzip, d.h. die Bibliotheken sollen für die Erledigung ihrer 
Bestellungen die Möglichkeiten der eigenen Leihverkehrsregion ausschöpfen.

Die projektinterne Fernleihe wird auch für den Aufbau von gedruckten Semesterapparaten genutzt. Unter Semesterapparaten versteht man eine Sammlung von Medien - im Falle des Projektes Lernraum sind es gedruckte Bücher - die für die Dauer eines Semesters in der Bibliothek zur Verfügung stehen. Diese Semesterapparate enthalten die Pflichtlektüre für ein Seminar. Während in deutschen Hochschulen Semesterapparate gern genutzt werden, spielt diese Form in tschechischen Hochschulen keine große Rolle und kommt eher in Ausnahmefällen vor. Frau Prof. Dr. Elke Mehnert, Professorin für Deutschsprachige Literatur in der Westböhmischen Universität in Pilsen, führte Semesterapparate für ihre Studierenden ein und nutzt dafür vorwiegend Bücher aus der Universitätsbibliothek Chemnitz.

Der Meilenstein 1 „Konsortiale Beschaffung der Medien/Fernleihe/Semesterapparate" stellt den wichtigsten Inhalt des Lernraum-Projektes. Alle Ziele wurden erreicht. Dazu zählt vor allem die Bildung des ersten sächsischtschechischen Konsortiums zum Erwerb elektronischer Medien. Dies wird auch nach dem Projektende die Nachhaltigkeit sichern und hat Vorbildwirkung für andere Bibliotheken. 


\section{MEDIENTECHNIK UND INFRASTRUKTUREN IM NETZWERK-LERNRAUM}

Falk Maiwald 


\subsection{Neuer Blick auf Lernräume wissenschaftlicher Bibliotheken}

Mit dem "Shift from Teaching to Learning” steht heute die Kompetenzentwicklung im Mittelpunkt der Lernaktivitäten von Studierenden. Zur klassischen Präsenzlehre in Vorlesungssälen oder Seminarräumen haben sich örtlich und zeitlich selbstbestimmte Formen des Lernens gesellt - sei es allein oder in der Gruppe. Hochschulen haben den Studierenden demnach einen erweiterten Rahmen und neue Bedingungen zu schaffen, um einerseits die Kompetenzziele ihrer Absolventinnen und Absolventen sicherzustellen, andererseits aber auch, um ihr Image positiv zu gestalten und im Wettbewerb um Studienanfänger erfolgreich bestehen zu können.

Dies gelingt mit einer "marktbezogenen" Gestaltung der Lernräume, insbesondere der Bibliotheken. Lernräume in bzw. von Bibliotheken sind als reale oder virtuelle Orte des Zusammentreffens von Angebot und Nachfrage zu verstehen. Auf der Angebotsseite wird ihre Gestaltung auch maßgeblich durch permanent weiter entwickelte Informations- und Medientechnologien beeinflusst. Die Entwicklung von Angeboten u.a. zur Digitalisierung in der Lehre (E-Learning, Lernmanagement-Plattformen), in der Zusammenarbeit (Webkonferenzen, Kollaborationstools) sowie in der Digitalisierung der Medien (E-Journals, E-Books, Datenbanken) werden entsprechend weiter vorangetrieben. Auf der Nachfrageseite stehen die Studierenden der sogenannten "Net-Generation“. Sie sind medienerfahren, können mit IT umgehen. Deshalb stellen sie per se hohe Ansprüche an die IT- und Medieninfrastrukturen der Bibliothek, die sie als reales, lokales Kommunikationszentrum und als Lernort „unter Gleichen“ nutzen wollen. Aber auch die Ansprüche anderer interner Nutzergruppen, wie Lehrende und Forschende, die im lokalen Lernraum effektiv und effizient Recherche- und weitere Arbeiten erledigen wollen, sind zu berücksichtigen.

Wissenschaftliche Bibliotheken konzentrieren sich bei der "marktbezogenen" technischen Ausgestaltung ihrer Lernräume auf die Anforderungen ihrer internen Nutzergruppen. Sie modernisieren ihre lokalen IT- Infrastrukturen für Einzelarbeitsplätze und für Gruppenräume. Seit vielen Jahren stehen solche Iokalen Angebote und Infrastrukturen auch externen Nutzern zur Verfügung. So öffnen auch die Projektpartner Studierenden, Lehrenden und Forschenden aus anderen Einrichtungen - auch aus dem jeweiligen Nachbarland - ihre Türen. 
Hier gilt es, den lokalen Lernraum gegebenenfalls an spezifische Bedürfnisse oder Ansprüche anzupassen und über die Vermittlung von Informationskompetenz (siehe Kapitel 5) dafür zu sorgen, dass die Externen die Angebote auch effektiv nutzen können.

Ein Aspekt wurde bei der Nutzerorientierung - sicher nicht nur der Projektbibliotheken - aber bislang wenig beachtet: Im "Markt" der wissenschaftlichen Bibliotheken sind solche Nutzergruppen identifizierbar, die mit anderen Bibliotheken oder den zugehörigen Universitäten oder Hochschulen in einer konkreten Beziehung und in Interaktion stehen. Mit anderen Worten gibt es interne und externe Nutzergruppen mit gemeinsamen Aufgaben oder Interessen. Dies können beispielsweise Projektstudien oder Forschungsseminare von Studierendengruppen, Forschungsprojekte von Wissenschaftlern, aber auch Wissens- und Erfahrungsaustausche unter Lehrenden verschiedener Universitäten oder Hochschulen sein. Vor allen Dingen finden die Mitarbeiterinnen und Mitarbeiter von Bibliotheken bilaterale oder multilaterale Ansatzpunkte im fachlichen Austausch oder in Projekten wie diesem hier. In der klassischen Form treffen sie sich dazu physisch präsent in realen, lokalen Lern- oder Arbeitsräumen.

Heute können aber mit Hilfe von Internettechnologien virtuelle Lern- und Arbeitsumgebungen als soziale Orte der Interaktion geschaffen werden, wobei auch politische und geografische Grenzen (im Regelfall) leicht überwunden werden können. Praktisch können Akteure auf der ganzen Welt solche virtuellen Lern- und Arbeitsräume nutzen. Wesentliche Vorteile sind:

- die hohe zeitliche Flexibilität,

- die niedrigere Schwelle für Austausche und Kollaboration und damit bestenfalls die Verbesserung von Projektergebnissen usw.,

- die positive Imagebeeinflussung für die Bibliotheken im Kontext Digitalisierung sowie

- der geringere Zeit- und Finanzaufwand durch erspartes Reisen.

Genau an dieser Stelle setzten die Vorüberlegungen des Projektes an. Die Projektpartner entwickelten damals erste grobe Aufgabenstellungen zur Gestaltung eines gemeinsamen grenzübergreifenden virtuellen Lern- und Arbeitsraums, der später als Netzwerk-Lernraum bezeichnet wurde, und bündelten diese im Meilenstein „Technische Ausstattung des Lernraums". 
Der virtuelle Netzwerk-Lernraum kann ähnlich einer Online-Community als ein informeller Zusammenschluss von Lernenden, Forschenden oder Lehrenden verstanden werden, die aufgrund gemeinsamer Interessen oder Problemstellungen über das Internet kommunizieren, kooperieren, Wissen und Erfahrungen austauschen und dabei voneinander lernen ${ }^{16}$. Dabei nutzen sie IT- und Medientechnik sowie weitere Infrastrukturen in den Bibliotheken. Im Vordergrund des Projektmeilensteins standen die Entwicklung, der Test und die Implementierung nutzergruppenspezifischer Sub-Netzwerk-Lernräume zwischen den Projektpartnern. Damit wurde das gemeinschaftliche Ziel verfolgt, die regionalen wissenschaftlichen Strukturen durch Vernetzung und Kooperation der jeweiligen Nutzergruppen zu stärken und grenzübergreifende individuelle Lern- und Forschungsprozesse zu initiieren und zu unterstützen. Als Nebeneffekt sollten die Bibliotheken ihr Image als moderne Medienzentren und Lernorte stärken bzw. verbessern.

Kernaufgabe in dem Meilenstein war es, die entsprechende technische Ausstattung bzw. die nötigen Infrastrukturen des Netzwerk-Lernraums für die Projektpartner zu planen und anzuschaffen. Dabei sollten auch Anforderungen der lokalen Lernräume der Projektpartner berücksichtigt werden, um Synergien und eine ökonomisch nachhaltige Nutzung zu realisieren. Voraussetzung für die technische Planung und Spezifizierungen war die Entwicklung bedarfsorientierter Anwendungsszenarien im angestrebten Netzwerk-Lernraum (vgl. Kapitel 3.2.). Dazu wurden mögliche Konstellationen der standort- bzw. grenzübergreifenden Zusammenarbeit in Webkonferenzen, multimedialen Präsentationen u.a. zwischen den Projektpartnern simuliert. Aus diesen Szenarien konnten dann die benötigten technischen Infrastrukturen abgeleitet, die technischen Parameter und Anforderungen spezifiziert und letztlich die Anschaffung und Installation der IT und der Medientechnik realisiert werden (Kapitel 3.3.).

Damit eine technische Neuerung getestet und später im täglichen Einsatz auch effektiv genutzt wird, braucht sie vor allem Akzeptanz und Übung der potenziellen Nutzer. Zu diesem Zweck wurden im Meilenstein verschiedene gemeinsame Aktivitäten geplant und durchgeführt, insbesondere der initiale Workshop in Pilsen im Januar 2017 sowie die Praxiswoche Liberec-Görlitz im Mai 2017 (siehe Kapitel 3.4). Aber auch weitere Gelegenheiten zur Nutzung und Demonstration der Technik in den lokalen Lernräumen der Projektpartner

${ }^{16}$ Kupfer (2015), S. 204. 
und im Netzwerk-Lernraum wurden ergriffen. Beispielhaft zu nennen sind der konsequente Einsatz der Technik bei den monatlichen Projekt-Webkonferenzen der Partner sowie das sehr erfolgreiche virtuelle Seminar zur Steigerung der Informationskompetenz im April 2018. Darüber hinaus wurden weitere technische Möglichkeiten nutzbar gemacht, die die Projektarbeit sinnvoll unterstützten. Auch begleitende Unterlagen wie zweisprachige Gebrauchsanleitungen wurden entwickelt.

\subsection{Prinzipielle Nutzungsszenarien für den neuen Netzwerk-Lernraum}

In einem wie hier definierten Netzwerk-Lernraum findet im engeren Sinn eine Kommunikation bzw. Kollaboration in Echtzeit statt. Das bedeutet, dass Nutzergruppen zwischen den Bibliotheken in Webkonferenzen und mit Hilfe interaktiver Kollaborationstools "live" miteinander diskutieren, Projekte bearbeiten und Konzepte entwickeln, deren Ergebnisse elektronisch verarbeitet werden. Im weiteren Sinn schließt ein Netzwerk-Lernraum auch eine zeitversetzte Kommunikation bzw. Kollaboration mit ein. Diese kann nacheinander bzw. wechselseitig erfolgen, beispielsweise über Projektmanagement-Tools, Dokumenten-, Wissensmanagement- oder E-Learning-Systeme oder auch über zentrale Datenspeicher zur Dateiablage.

Im Vordergrund dieses Projektmeilensteins stand - wie eingangs in Kapitel 3.1. beschrieben - die Entwicklung möglicher Nutzungsszenarien für eine Live-Kommunikation unter Berücksichtigung der lokalen Lernraum-Technik.

\section{Nutzung durch die Bibliotheksmitarbeiter}

Die Bibliotheken können die Möglichkeiten des Netzwerk-Lernraums für ihre eigenen Zwecke nutzen. Beispielsweise kann die Gruppe der Bibliothekare Workshops oder Schulungen zur Beschaffung von E-Medien oder Austausche über die Vermittlung von Informationskompetenz grenz- bzw. standortübergreifend durchführen. Die Leitungen kooperierender Bibliotheken können interaktive Webkonferenzen über strategische Ausrichtungen und gemeinsame Aktivitäten führen.

Darüber hinaus sind zum Beispiel Schulungs-, Beratungs- und Workshopangebote für Studierende, Lehrende und Forschende denkbar, die nicht vor Ort präsent sein können, also beispielsweise ausländische Nutzer. 


\section{Nutzung durch Studierende, Forschende, Lehrende}

Forscher und Lehrende können die Bibliothek bzw. ihre Ausstattung als Netzwerk-Lern- und Arbeitsraum buchen und nutzen. Angebote für Fachkollegen zur Kollaboration in Forschungsthemen, aber auch Öffentlichkeitsarbeit vor Firmen oder im Nachbarland, virtuelle Workshops, Beratungen von Gremien sind denkbar. Hervorzuheben sind hier besonders solche Interaktionen, die über eine normale Webkonferenz hinausgehen, beispielsweise, wenn gleichzeitig von zwei oder mehreren Standorten an demselben gemeinsamen Dokument gearbeitet wird. Zweckmäßig erscheint auch die Nutzung durch Studierende und ihre Betreuer, wenn beispielsweise Forschungsarbeiten oder Abschlussarbeiten mit entfernten nationalen oder internationalen Unternehmen zu besprechen sind. Auch könnten sich Forschende oder Lehrende deshalb in die Bibliothek „einmieten“, weil die räumlichen Bedingungen in der Bibliothek vorteilhafter sind.

Diese Szenarien lassen sich mit Beispielen und Ideen theoretisch weiter ausbauen. Im Laufe des Projektes wurden solche und weitere praktische Anwendungsmöglichkeiten demonstriert bzw. genutzt (siehe Kapitel 3.4). Die hier vorgestellten Überlegungen genügten jedoch, um nachfolgend die wichtigsten Bedarfe und Anforderungen an die technische Ausstattung für die Projektpartner abzuleiten.

\subsection{Anforderungen, Anschaffung und Implementierung der neuen Medien- und IT-Technik}

Anhand der Szenarien wurde abgeleitet, welche wesentlichen Geräte und Infrastrukturen zur technischen Ausstattung erforderlich waren. Abbildung 1 skizziert das Prinzip der Live-Kommunikation im Netzwerk-Lernraum über das Internet sowie die wichtigsten technischen Geräte.

\section{Internetzugang - das standortverbindende infrastrukturelle Rückgrat}

Damit eine Kommunikation grenzübergreifend bzw. zwischen den Standorten ermöglicht werden kann, sind Breitband-Internet-Zugänge sowie Software zur Verbindung (Videokonferenzfunktion, Kollaborationsfunktion durch LiveZugriff) nötig. Diese Voraussetzungen waren in den Projektbibliotheken bzw. 


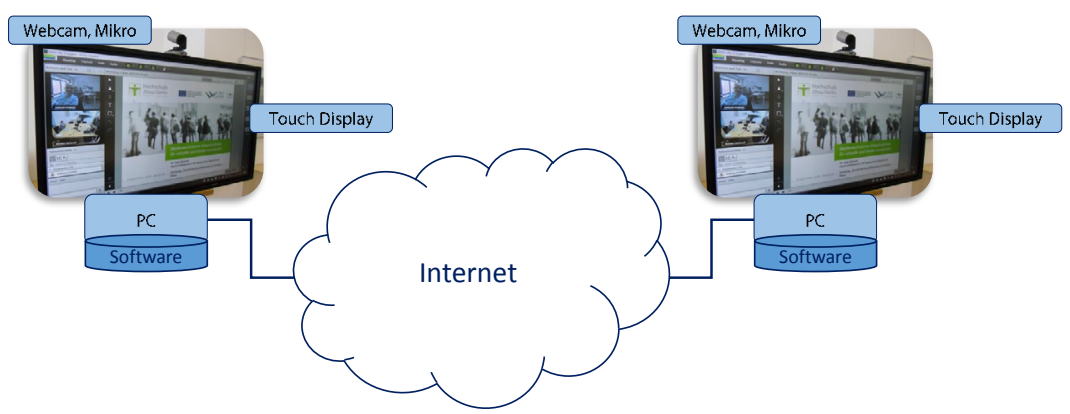

Abbildung 1. Prinzip des Live-Kommunikations-Systems im Netzwerk-Lernraum. Quelle: Eigene Darstellung; Foto: HSB Zittau/Görlitz.

durch die jeweiligen Hochschul- und Universitätsrechenzentren gegeben. In Deutschland sind die staatlichen Hochschulen über das Deutsche Forschungsnetz (DFN) ${ }^{17}$, in Tschechien über das Czech Education and Scientific NETwork (CESNET) ${ }^{18}$ mit sehr hohen Bandbreiten an das Internet angeschlossen. Die wissenschaftliche Bibliothek in Liberec verfügt über einen Breitband-Zugang durch Liberecka IS. Für den Netzwerk-Lernraum bieten diese Internetzugänge das standortverbindende infrastrukturelle Rückgrat.

\section{Konferenztools - die Software für Kommunikation und Interaktion}

Zur Realisierung der Online-Veranstaltungen stand zunächst die Software „Adobe Connect" zur Verfügung. In Deutschland ist diese für alle Hochschulen wiederum über das DFN, in Tschechien über CESNET lizensiert. Im Verlauf des Projektes wurde mit dem Tool WebEx der Firma Cisco, das jedoch nur die Technischen Universität Chemnitz lizensiert hat, eine weitere Möglichkeit erschlossen. Beide Tools ähneln sich; die Qualität der Übertragungen erschien jedoch in der Anwendung bei WebEx besser. Beide Tools haben gemein, dass lediglich ein Lizenzinhaber nötig ist, um die anderen Partner in die LiveKommunikation der Online-Veranstaltungen einladen zu können. Im Szenario der Bibliothekszusammenarbeit war und ist dies für die Wissenschaftliche Bezirksbibliothek Liberec besonders relevant, da sie keinerlei entsprechende

${ }_{17}$ Das DFN wird vom Verein zur Förderung eines Deutschen Forschungsnetzes e.V. betrieben, vgl. DFN (2018).

${ }^{18}$ Das CESNET wird von CESNET, z. s. p. o. betrieben, vgl. CESNET (2018) https://www.cesnet.cz/. 
Lizenz besitzt. Beispielsweise können aber auch Firmen in den Genuss dieser Möglichkeit kommen und sich für Projekte und Besprechungen mit Lehrenden und Forschenden in den Netzwerk-Lernraum einwählen.

\section{Computer- und Medientechnik - die lokale Hardware-Infrastruktur}

Wissenschaftliche Bibliotheken sind im lokalen Lernraum bereits mehr oder weniger mit moderner Computer- und Medientechnik ausgestattet. Einzelarbeitsplätze in Carrels bzw. Arbeitskabinen werden regelmäßig mit feststehender IT-Ausstattung (typisch: PC mit Bildschirm, ggf. Scanner) versehen. Im BYOD-Trend ${ }^{19}$ führen die Nutzer ihre Endgeräte mit, die sie dann sowohl in Carrels, als auch an Arbeitsplätzen im freien Raum für Stillarbeit oder für Kurzrecherchen nutzen. Entsprechend sind ein leistungsfähiges WLAN sowie Stromanschlüsse bereitzustellen.

Die für den Netzwerk-Lernraum interessanten Gruppenarbeiten finden innerhalb der Bibliothek in extra dafür ausgestatteten Räumen oder Bereichen statt. Sowohl fest installierte Computer, als zunehmend auch BYOD-Infrastrukturen sind gebräuchlich. Als Medientechnik dienen klassisch Beamer und Tonanlagen. Im Zuge der Digitalisierung treten zunehmend interaktive Whiteboards an die Stelle von Tafeln, Flipcharts oder Beamern. Eine Unterart dieser interaktiven Whiteboards sind Präsentations- und Interaktionsdisplays, sogenannte Touch Displays. Als Interaktionsdisplays verfügen sie über eigene Computer- und Netzwerktechnik. Sie lassen sich aber auch durch externe Personal-Computer (PC), Laptops oder mobile Endgeräte mit MiracastFunktionen ${ }^{20}$ zu Präsentationszwecken ansteuern.

Grundsätzlich sind für internetbasierte Live-Kommunikation bzw. OnlineVeranstaltungen mindestens ein PC/Laptop mit W/LAN-Zugang, ein Bildschirm und/oder Beamer, eine Webcam und ein Mikrofon erforderlich. Für interaktive Gruppenarbeiten eignen sich Touch Displays anstelle von klassischen Bildschirmen oder Beamern besonders. Die sehr großen Displays

19 „Bring Your Own Device“ beschreibt den Zugriff auf die IT-Infrastruktur einer Organisation mit Hilfe organisationsfremder Geräte, vgl. DINI (2013), S. 65. Solche Geräte sind insbesondere Notebooks, Tablet-PCs und Smartphones.

${ }_{20}$ Miracast ist ein Standard, der es ermöglicht, Bildschirminhalte von Smartphones oder Tablet-PCs auf größere Displays zu übertragen. 
(65" und mehr) werden in geeigneten Gruppenarbeitsräumen aufgestellt. Idealerweise wird das Technik-Portfolio durch eine extra für solche Räume ausgelegte hochauflösende Webcam und ein hochwertiges Konferenzmikrofon komplettiert.

\section{Auswahl und Anschaffung der technischen Ausstattung}

Anspruch des Projektes war es, die Anforderungen der Nutzer an die Ausstattung in lokalen Lernräumen (siehe Kapitel 3.1) mit denen des NetzwerkLernraums zu verbinden. Ausgehend von den in Kapitel 3.2. beschriebenen Szenarien beschlossen drei Partner im Projekt die Anschaffung von Touch Displays mit der adäquaten Konferenztechnik. Darüber hinaus wurde auch von den anderen beiden Projektpartnern dem Projektzweck dienende Computerund Medientechnik beschafft.

Die Spezifizierung der technischen Anforderungen gelang durch umfangreiche Marktrecherchen und diverse Beratungsgespräche mit Spezialisten. Hier zeigte sich, dass die entwickelten Szenarien hilfreich für das Verständnis der Technik-Anbieter bezüglich der Gesamtidee des Lernraums waren. Bei den Displays lag ein besonderes Augenmerk auf der Mobilität, sodass die Technik bei Bedarf in verschiedenen Räumen genutzt werden kann. Letztlich folgte ein üblicher Ausschreibungs-, Vergabe- bzw. Beschaffungsprozess über die zentralen Einrichtungen der Projektpartner.

Der Leadpartner, die Universitätsbibliothek der Technischen Universität Chemnitz, beschaffte ein Touch Display Galneoscreen 65" mit höhenverstellbaren Fahrgestell sowie Swift-Turn-Table, das heißt einem klappbaren Tisch für Laptop/Tastatur. In dem neu entstehenden Bibliotheksbau kann das Display dank seiner Größe und Mobilität später weiterhin dem Projektzweck entsprechend genutzt werden.

Die Hochschulbibliothek Zittau/Görlitz erwarb für ihre beiden Standorte jeweils ein Touch Display Promethean ActivPanel, 65“ mit höhenverstellbarem Fahrgestell, eingebautem PC und Extron Sharlink zum lokalen Miracast für bis zu 4 Teilnehmer (siehe Abbildung 2). Mit dem Logitech ConferenceCam Kit wurden eine sehr leistungsfähige USB-Webcam und ein Konferenzmikrofon eingeführt. Die Geräte wurden jeweils in einem großen Gruppenarbeitsraum aufgestellt. 


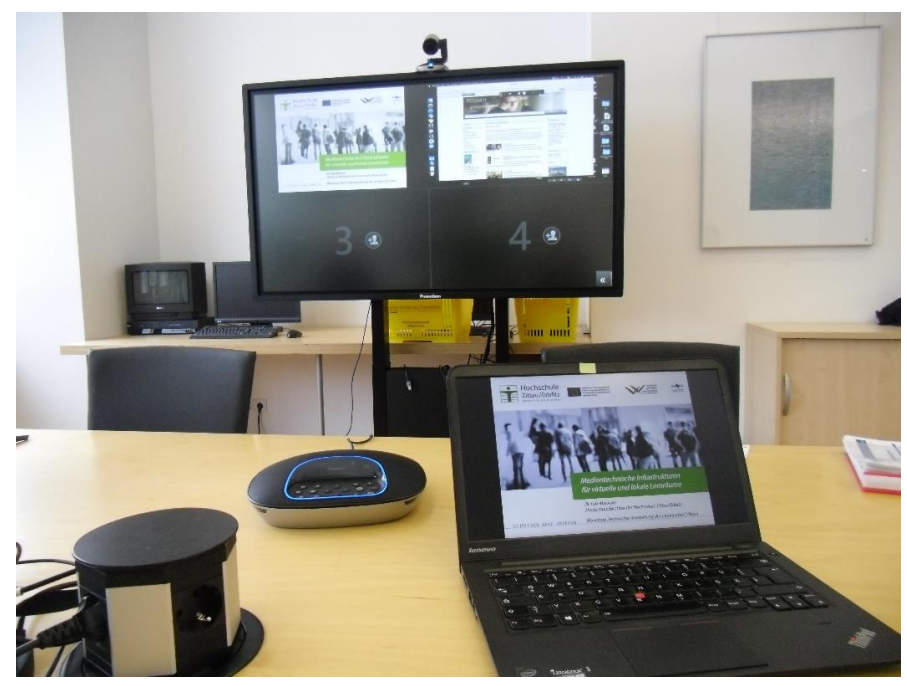

Abbildung 2. Beispiel für Miracast mit Laptop und ActivPanel im Gruppenarbeitsraum. Foto: HSB Zittau/Görlitz.

Die Universitätsbibliothek der Westböhmischen Universität in Pilsen kaufte aus Projektmitteln sechs Notebooks HP ProBook 640 G2 sowie einen Beamer Epson EB-535W+Epson Wall Mount ELPMB45. Genutzt wird die Technik bei Schulungen und Seminaren für die Nutzer im lokalen Lernraum sowie für den Einsatz bei den projektbezogenen Seminaren, Workshops und Webkonferenzen.

Die Wissenschaftliche Bezirksbibliothek in Liberec erwarb für den großen Konferenzsaal einen leistungsfähigen Projektor sowie eine Leinwand, die für Vorträge, Konferenzen und Filmprojektionen eingesetzt werden. Darüber hinaus wurde eine spezielle Kamera mit Software installiert, die sowohl Aufnahmen im Raum, als auch Übertragungen in den virtuellen Raum, beispielsweise auch auf YouTube, ermöglicht.

Die Universitätsbibliothek der Technischen Universität in Liberec erwarb zwei Laptops und einen mobilen Beamer, der bedarfsweise im lokalen Lernraum eingesetzt wird. Wie auch beim Leadpartner und der Hochschulbibliothek Zittau/Görlitz wurde ein interaktives Touch Display (ActivBoard) mit Fahrgestell sowie passendes Videokonferenz-Zubehör, unter anderem ein mobiles Audiosystem, ein drahtloses Mikrofon und eine Full-HD-Kamera, beschafft. 


\section{Weitere Tools im Netzwerk-Lernraum}

Wie in Kapitel 3.2. beschrieben, kann ein Netzwerk-Lernraum auch Tools für eine zeitversetzte Kommunikation bzw. Kollaboration nutzen. Dazu zählen spezielle E-Learning-Plattformen wie Moodle (siehe Kapitel 5) und Tools zur Ablage von Projektdokumenten und ähnlichem.

Bereits zu Beginn des Projektes vereinbarten die Projektpartner, dass die Projektarbeit für alle Partner transparent und nachvollziehbar dokumentiert werden muss und die Dokumente und andere Dateien an einer für alle Partner geschützt zugänglichen Stelle elektronisch abgelegt sein sollen. Im Zeitalter cloudbasierter Angebote diverser Anbieter im Internet ist eine zentrale Dokumenten- bzw. Dateiablage leicht umsetzbar. Unter Sicherheitsaspekten sind solche Angebote jedoch kritisch zu betrachten. Insofern bestand die Aufgabe, eine eigene sichere Projekt-Cloud aufzubauen. Durch die Fachkompetenz und die entsprechenden IT-Ressourcen des Hochschulrechenzentrums der Hochschule Zittau/Görlitz (siehe zur Rolle der Rechenzentren Kapitel 3.5) konnte mit der Software "Filr" der Firma Micro Focus eine zuverlässige Lösung implementiert werden. Die Projektmitarbeiter aller Partner konnten mit individuellen Zugriffsrechten über die Filr-WebOberfläche, über das Filr-Programm für PC/Laptop oder über die Filr-App für mobile Geräte jederzeit auf die Projekt-Cloud und die Inhalte zugreifen.

\subsection{Einsatz der Technik im grenzübergreifenden Netzwerk-Lernraum}

Eine Vielzahl an Aktivitäten, wie Demonstration der technischen Möglichkeiten, Schulungen, Entwicklung von verständlichen Gebrauchsanweisungen und letztlich die konsequente Einbeziehung in die Projektarbeit waren Schlüssel für eine erfolgreiche Implementierung und Nutzung der angeschafften Technik und der Softwaretools.

\section{Workshop in Pilsen - erste Ansätze für den technischen Netzwerk-Lernraum}

Beim Workshop "Technische Ausstattung des Lernraums" im Januar 2017 in Pilsen stellten alle Projektpartner ihre lokalen Infrastruktur- und Technikbedingungen vor. Um die Ansätze und Nutzenvorteile der technischen 
Anschaffungen zunächst allen beteiligten Projektmitarbeitern zu verdeutlichen, führte die HSB Zittau/Görlitz in die möglichen Nutzungsszenarien ein. Mit einer Live-Demonstration zwischen Pilsen und Zittau konnten die Leistungsfähigkeit der Konferenzsoftware und -technik und die Interaktionsmöglichkeiten vorgestellt werden. Dabei wurden das in Zittau bereits implementierte Touch Display und die periphere Konferenztechnik einbezogen.

\section{Praxiswoche Liberec-Görlitz - Schwerpunkt IK-Modul und Webkonferenz mit allen Partnern}

Die beiden Projektbibliotheken in Liberec und die Hochschulbibliothek am Standort Görlitz richteten die Praxiswoche in dem Meilenstein im Mai 2017 aus. Schwerpunkt dieser Projekttage war die Frage, wie ein Modul zur Informationskompetenz inhaltlich konzipiert und technisch bzw. medial untersetzt werden kann. Entsprechend wurden Aspekte des E-Learnings aufgegriffen und mit Fragen der Vermittlung von Informationskompetenz verbunden (siehe zu diesem Meilenstein Kapitel 5).

Die angeschaffte Computer- und Medientechnik kam hier sowohl bei der lokalen Gruppenarbeit der Projektmitarbeiter, als auch bei der etwa einstündigen Abschlusspräsentation zum Einsatz, die von Görlitz aus als Webkonferenz mit allen Projektstandorten geteilt wurde. Dabei kamen die Vorzüge dieser Lösung für eine virtuelle Bibliothekszusammenarbeit deutlich zum Tragen, denn neben den an der Praxiswoche Beteiligten konnten die Mitarbeiter, die Leitungen sowie weitere Gäste an den entfernten Standorten an der Präsentation teilhaben.

\section{Regelmäßige Online-Meetings - ein neuer Weg der Projektarbeit}

Aus der positiven Erfahrung der Praxiswoche entwickelte sich ein neuer, turnusmäßiger Weg der Besprechung und Koordination in der Projektarbeit. Mit Start am 12. Juni 2017 wurde fortan monatlich ein Onlinemeeting aller Projektpartner durchgeführt. Dank der angeschafften Technik und dem Tool Adobe Connect - später WebEx - konnten Projektfragen beantwortet und Aufgaben besprochen werden. Dies war nicht nur hoch effizient, sondern steigerte durch die Regelmäßigkeit auch das Involvement der Projektpartner. Bis Juni 2018 wurden zwölf Onlinemeetings durchgeführt. 


\section{Lokale Nutzung der Technik}

Wie bis hierhin dargestellt, wurde innerhalb der "Projektgemeinde" viel unternommen, um die Technik im Projektsinn und darüber hinaus für die Bibliotheksarbeit und den Austausch zu nutzen. Beispielhaft sei hier noch angeführt, dass die Wissenschaftliche Bezirksbibliothek in Liberec eine Übertragung der Abschlussveranstaltung des Projektes via YouTube plant.

Aber auch die in Kapitel 3.2. gezeigten Nutzungsszenarien für andere Nutzergruppen, wie Studierende, Lehrende und Forschende, wurden aufgegriffen. Hier lag der Fokus auf der lokalen Nutzung der Technik. Dazu war es vorab notwendig, die Mitarbeiter in den Bibliotheken im Umgang mit der neuen Technik zu schulen, damit diese durch die Bibliotheksnutzer verstanden und angenommen werden. Passend wurden von einigen Projektpartnern entsprechende Tutorials, beispielsweise für die vielen Funktionen und Möglichkeiten der Touch Displays, entwickelt und in deutscher und tschechischer Sprache für die Nutzer gedruckt.

Der Erfolg hat sich nach und nach eingestellt. Alle Projektpartner resümieren, dass die angeschaffte Technik von den Studierenden sehr gut angenommen und genutzt wird. So werden beispielsweise am ActiveBoard der Technischen Universität in Liberec verschiedene interaktive Apps für Lehre/Studium sowie Lehrvideos verwendet und der Bibliotheksraum mittels Relax-Musik und Werbevideos aufgewertet.

\section{Virtuelles Seminar - andere Nutzergruppen heranführen}

Möglichkeiten der interaktiven standortübergreifenden Lern- und Arbeitsräume mussten - wie vorher auch bei den eigenen Projekt- und Bibliotheksmitarbeitern - für die anderen Nutzergruppen überhaupt erst sichtbar und erlebbar gemacht werden. Zu diesem Zweck führten die Projektpartner unter Federführung der HSB Zittau/Görlitz im April 2018 ein virtuelles Seminar durch, zu dem Lehrende, Forschende und Studierende in die jeweilige „Heimatbibliothek" mit entsprechender Werbung eingeladen wurden. Mit Einsatz der Technik und über WebEx wurde wieder eine Webkonferenz zwischen den Projektstandorten Chemnitz, Pilsen, Liberec, Zittau und Görlitz aufgebaut. Im Publikum fanden sich an allen Standorten zahlreiche Teilnehmer aus allen relevanten Nutzergruppen. 
Das Besondere an dem Seminar war, dass Ergebnisse dieses und der anderen Meilensteine vorgestellt und dem Publikum quasi das gesamte Erfolgsspektrum des Projekts Lernraum - bibliothekarische Informationsplattform demonstriert werden konnte. So trugen die Partner nacheinander Möglichkeiten der Mediennutzung und der Fernleihe vor. Die konsortial beschafften E-Books wurden präsentiert und beworben. Am Ende des Seminars wurden die Interaktionsmöglichkeiten für Gruppenarbeiten zwischen den Standorten live an den Touch Displays vorgeführt.

Das positive Feedback zu dieser Veranstaltung zeigte eindrucksvoll, dass die Projektergebnisse sehr relevant und nützlich für viele Nutzer sein können.

\subsection{Lessons learned}

In den vorangegangenen Kapiteln wurden bereits wichtige Erfolgsfaktoren für die Planung, Anschaffung und die erfolgreiche Nutzung der Technik beschrieben.

Ein weiterer wichtiger Erfolgsfaktor war die Unterstützung aus den Rechenzentren bzw. IT-Abteilungen der Projektpartner. Die fachliche Expertise war für die Planung, die Bereitstellung und den Betrieb der Medientechnik, der IT und der entsprechenden Infrastrukturen unerlässlich. Den IT- und MedientechnikExperten der Partnerbibliotheken kam also eine entscheidende Rolle bei der Überführung der technischen Gestaltungs- und Anwendungsideen in zweckmäßige Konzepte und deren Umsetzung zu. Konfigurationsvarianten wurden geplant und miteinander verglichen. Die Projektpartner tauschten sich intensiv über die geplanten Anschaffungen aus. Im Zuge des Aufbaus der virtuellen Arbeitsräume wurden diverse Tests durchgeführt. Auch bei den erstmaligen Anwendungen der neuen Technik im Echtbetrieb war die Unterstützung der IT-Experten unverzichtbar.

Im Fazit ist zu konstatieren, dass mit der Beschaffung und Nutzung der Computer- und Medientechnik die Arbeit der Projektbibliotheken untereinander sowie die Angebotspalette für die Nutzer der Bibliotheken auf ein technologisch neues Level gehoben wurden, das voll im Digitalisierungstrend liegt und damit in die Zukunft weist. 


\section{Literatur}

Kupfer, F.: Lernen und Arbeiten in Lernort übergreifenden Netzwerken, in: Wittwer, W.; Diettrich, A.; Walber, M. (Hrsg.): Lernräume, Wiesbaden 2015, S. 204-217.

DINI, Deutsche Initiative für Netzwerkinformation e.V.: Die Hochschule zum Lernraum entwickeln, Kassel 2013.

DFN, Verein zur Förderung eines Deutschen Forschungsnetzes e.V.,

URL: https://www.dfn.de/xwin/, zuletzt zugegriffen am 16.08.2018.

CESNET, z. s. p. 0., Czech Education and Scientific NETwork,

URL: https://www.cesnet.cz/, zuletzt zugegriffen am 16.08.2018. 



\section{OPEN ACCESS}

Zdeňka Firstová

Miroslava Pourová 


\subsection{Grundlagen}

Jede wissenschaftliche Arbeit beginnt mit dem Studium von Fachliteratur, vor allem von Artikeln in rezensierten wissenschaftlichen Zeitschriften. Ein Widerspruch ist dabei, dass trotz des Anstiegs der Publikationen, deren Zugänglichkeit durch den Verdienst der immer weiter wachsenden Preise für Zeitschriften, schlechter wird. Am meisten belastet diese Tatsache die Wissenschaftler, welche gezwungen sind, im Hinblick auf die Art und Weise der Bewertung der Wissenschaft in Zeitschriften zu publizieren. Die großen kommerziellen Verleger, welche Prestigezeitschriften herausgeben, erhöhen die Preise fürs Abonnement der Zeitschriften und der Datenbank, welche die Titel zugänglich machen. Die Autoren der Artikel, welche ohne Anspruch auf Honorar veröffentlicht werden, kommen dadurch oft nicht an die Arbeiten ihrer Kollegen, weil sich deren Institutionen die Anschaffung sämtlicher notwendigen Zeitschriften nicht leisten können. Alarmierend ist, dass dies inzwischen auch die reichsten Universitäten betrifft. Bereits im Jahr 2012 machte der Direktor der Bibliothek der HarvardUniversität Robert Darnton auf diese widersprüchliche Situation aufmerksam: Die Mitglieder der akademischen Gemeinde führen eine Untersuchung durch, schreiben darüber ohne Vergütung, rezensieren die Arbeiten ihrer Kollegen, arbeiten in Editionsräten mit und müssen sich dann die Ergebnisse ihrer Arbeit zu überhöhten Preisen zurückkaufen. ${ }^{21}$

Ein weiterer Widerspruch besteht darin, dass Wissenschaft und Forschung zu einem großen Teil mit öffentlichen Geldern finanziert, aber deren Ergebnisse in kommerziellen Zeitschriften publiziert werden und der Zugang dazu abermals aus öffentlichen Ressourcen bezahlt wird. Einige Akademiker sprechen daher im Zusammenhang mit dem Publizieren von einer Krise der traditionellen wissenschaftlichen Zeitschriften. Außer dem Problem mit dem Preis und mit der Zugänglichkeit, weist eine Reihe von Autoren auch auf den zeitlichen Verzug hin. Zwischen dem Verfassen des Artikels und dessen Herausgabe in einer klassischen Zeitschrift vergeht eine lange Zeit. Besonders bei renommierten Titeln verursacht eine Verzögerung nicht nur ein langes Rezensionsverfahren, sondern auch einen Überschuss an angebotenen Arbeiten, sodass solche Zeitschriften für manche Fächer keine Quelle für neue Informationen mehr sind.

${ }^{21}$ https://www.theguardian.com/science/2012/apr/24/harvard-university-journal-publishersprices 
Die Initiative Open Access (OA) treibt den Gedanken voran, dass die Ergebnisse von Wissenschaft und Forschung sofort und kostenlos zugänglich sein sollten, und bemüht sich, diese Widersprüche zu beseitigen.

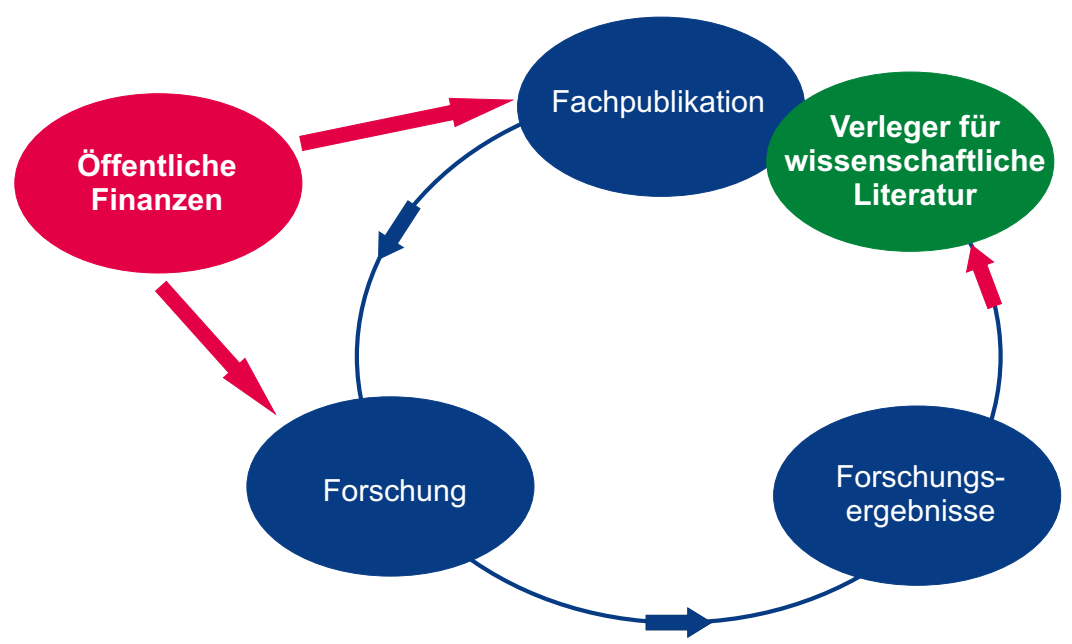

Abbildung 1. Wissenschaftliche Arbeit.

Die Prinzipien des Open Access wurden Anfang dieses Jahrhunderts durch drei Initiativen formuliert: Budapest Open Access Initiative (Februar 2002), Bethesda Statement on Open Access Publishing (Juni 2003) und Berlin Declaration on Open Access to Knowledge in the Science and Humanities (Oktober 2003), zusammenfassend bezeichnet man sie als BBB-Initiativen. Kurz gesagt gewährleistet das Open Access (offener Zugang) der wissenschaftlichen Öffentlichkeit einen kostenlosen und dauerhaften Zugang zu Fachtexten im Internet. Dadurch beseitigt es die Ungleichheit im Zugang zu Informationen und verändert grundsätzlich das Modell der Finanzierung der wissenschaftlichen Veröffentlichung. Die im OA veröffentlichen Texte können ohne besondere rechtliche, finanzielle oder andere Einschränkungen weiter kopiert, reproduziert, gedruckt, vervielfältigt und durchgeschaut werden. Die Initiative ist zugleich eine Reaktion auf den bedeutenden Einfluss des Internets auf dem Gebiet der Verbreitung von Informationen und wissenschaftlichen Erkenntnissen und bietet eine Alternative zum klassischen Modell des Publizierens bei den kommerziellen Verlegern. 
Eine der Möglichkeiten, wie man einen offenen Zugang zu Publikationen sicherstellt, ist der so genannte goldene Weg, wenn der offene Zugang vom Herausgeber gewährleistet wird. Die Art und Weise, wie er dies ermöglicht, ist vielfältig. Es existieren $0 A$-Zeitschriften, welche frei zugänglich sind, d.h. deren gesamter Inhalt ist jedermann kostenfrei zugänglich. Die Zeitschriften werden entweder durch Stiftungen oder Organisationen finanziert (oft handelt es sich dabei um Universitäten) und das Publizieren darin ist entweder kostenfrei oder man muss für die Publikation eines Artikels eine APC-Gebühr (article publishing charge oder woanders auch article processing charge genannt) entrichten. Die Liste der über 11500 Open Access-Zeitschriften ist im Adressverzeichnis DOAJ - Directory of Open Access Journals zu finden. ${ }^{22}$

Dank der Tatsache, dass das Open Access bereits seit fast zwei Jahrzehnten tätig ist, gab eine Reihe von klassischen wissenschaftlichen Zeitschriften dem ausdauernden Druck nach und ermöglicht den Autoren gegen eine APC-Gebühr die Veröffentlichung eines konkreten Textes, welcher anschließend frei verfügbar ist. Da in diesen Zeitschriften nebeneinander offene und nur für Abonnenten zugängliche Artikel zu finden sind, nennt man sie hybride Zeitschriften. Außerdem existieren auch Zeitschriften, welche die Artikel nach einer bestimmten Zeitspanne zugänglich machen.

Einige Universitäten stellten zur Unterstützung des goldenen OA-Weges finanzielle Mittel zur Errichtung von Fonds bereit, durch den die Gebühren gedeckt werden. Da die finanziellen Mittel nicht unbegrenzt sind, wird der Fonds meist ausgeschöpft, ohne dass das Interesse aller Interessenten befriedigt wird. Daher werden diverse Kriterien zur Erlangung eines Beitrags am APC festgelegt, z.B. die Forderung eines bestimmten Wertes des Impaktfaktors bei der Zeitschrift, die Höhe des maximalen Zuschusses, maximal ein Antrag pro Autor u.ä.

Eine zweite Möglichkeit des offenen Zugangs ist der so genannte grüne Weg. Diese Form wird vom Autor selbst gewährleistet, indem er seine Arbeit in dem Open Access-Repositorium speichert, d.h. in einem geschützten digitalen Speicherplatz, welcher einen dauerhaften Zugang zu den dort gespeicherten Publikationen gewährleisten muss. Im Prinzip unterscheiden wir institutionelle Repositorien (etwa $85 \%$ ), die in der Regel von Universitäten und anderen wissenschaftlichen Institutionen angelegt werden; fachliche Repositorien,

22 www.doaj.org 


\section{Typen des Open Access}

\section{Goldener Weg}

\section{Offener Zugang wird vom Verleger sichergestellt}

Zeitschriften mit voll offenem Zugang = der ganze Inhalt wird augenblicklich allen kostenfrei zugänglich gemacht

Open Access mit zeitlichem Embargo = volle Texte werden nach Ablauf einer vom Verleger festgesetzten Frist zugänglich gemacht

\section{Grüner Weg}

\section{Offener Zugang wird vom Autor sichergestellt}

Der Autor lädt seine in einer klassischen Zeitschrift / einem klassischen Sammelband veröffentlichte Arbeit in die offene Datenbank

\footnotetext{
Hybride Zeitschriften = ermöglichen nach der Entrichtung einer Publikationsgebühr die Zugänglichkeit des Artikels; hier gibt es nebeneinander Artikel mit bezahltem und offenem Zugang
}

\section{Abbildung 2. Schema der OA-Typen.}

in denen Publikationen nach ihrer inhaltlichen Ausrichtung veröffentlicht werden, oder Repositorien für eine bestimmte Dokumentenart (elektronische Dokumente, digitalisierte Dokumente, Daten).

Das älteste und bekannteste fachliche Repositorium ist $\operatorname{arXiv}^{23}$, dessen Anfänge zum Beginn der 90er Jahre zurückreichen. Es wurde ursprünglich zum schnellen Austausch wissenschaftlicher Informationen für die Physiker angelegt. Heute umfasst es Preprints aus der Physik, der Mathematik, der Computerwissenschaft, der quantitativen Biologie, den Finanzen und der Statistik.

Die Liste der Repositorien mit Angaben über Institution, Betreiber, Land, Repositoriumstyp, inhaltliche Ausrichtung, Anzahl der enthaltenen Dokumente usw. mit der Möglichkeit des Filterns und Heraussuchens bietet The Directory of Open Access Repositories - OpenDOAR ${ }^{24}$. Institutionen können sich auch an das Repositories Support Project ${ }^{25}$ wenden, wo sie Hilfe beim Anlegen eines Repositoriums finden.

\footnotetext{
${ }^{23}$ https://arxiv.org

24 www.opendoar.org

25 www.rsp.ac.uk
} 
Die wachsende Bedeutung des Open Access wird auch durch die Tatsache bestätigt, dass sich während der letzten zehn Jahre die Anzahl von OA-Zeitschriften und Repositorien verdoppelt hat. Gegenwärtig sind im DOAR über 3500 Repositorien aufgeführt. Deutschland steht mit 204 Repositorien weltweit an vierter Stelle (hinter den USA, Großbritannien und Japan). Die Tschechische Republik hat hier 18 registrierte Repositorien. ${ }^{26}$

Heute verfügt die Mehrheit der renommierten kommerziellen Verlage über eine ausformulierte OA-Politik und ermöglicht den Autoren, ihre Publikationen in irgendeiner Form in die offenen Repositorien einzustellen. Obwohl es sich bei den Autoren der Artikel um Wissenschaftler handelt, werden deren Rechte, über ihre Werke zu verfügen, durch die meisten Lizenzverträge mit den Verlegern in beträchtlicher Weise eingeschränkt. Der Autor überlässt seine Nutzungsrechte bei der Veröffentlichung in der Regel dem Verleger. Mit einer ergänzenden Vereinbarung zum Verlagsvertrag kann eine Zweitverwertung im Open Access ermöglicht werden. ${ }^{27}$ Durch die Vergabe von Open Access-Lizenzen können Urheber verschiedene Nutzungsrechte für ihre Werke einräumen. In Wissenschaft und Forschung werden häufig Creative Commons Lizenzen (CC) als Standard verwendet. ${ }^{28}$

Zur Erleichterung der Orientierung des Autors in der Verlagspolitik der Zeitschriften dient die Datenbank SHERPA/ROMEO. Dort sind die Open Access-Policies von Verlagen verzeichnet. Das Farbschema grün, blau, gelb und weiß kennzeichnet, welches Format für eine Open Access-Veröffentlichung genutzt werden darf.

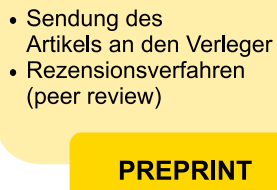

Abbildung 3. Versionen nach Veröffentlichungsstatus.

\footnotetext{
${ }^{26}$ Daten vom 11.05.2018.

27 Das Muster ist auf http://sparc.arl.org/ verfügbar.

${ }^{28}$ Alle nationalen Versionen der CC-Lizenzen stehen auf http://www.creativecommons.cz bzw. https://de.creativecommons.org/ zur Verfügung.
} 


\subsection{Vorteile und Schwierigkeiten beim Open Access}

Der Vorteil eines offenen Zugangs für die Leser liegt klar auf der Hand; aber befassen wir uns mit dem Vorteil für den Autor. Die Statistiken zeigen, dass die im $0 A$ veröffentlichen Artikel sichtbarer sind und daher häufiger zitiert werden. Zugleich erhält der Autor ein schnelles Feedback auf seine Arbeit, da die Kommunikation mit den Kollegen aus der ganzen Welt schneller und flexibler ist. Es kommt somit zu einer Erleichterung der Zusammenarbeit und sogar zu einer breiteren Einbindung der Wissenschaftler aus anderen Fachgebieten, sodass sich eine neue Sicht auf die gegebene Problematik auftut. Das Open Access bringt auch anderen Beteiligten im Prozess der wissenschaftlichen Veröffentlichung einen Vorteil: Der Herausgeber der Zeitschrift gewinnt mehr Leser; eine höhere Zahl an Zitaten aus den Artikeln bringt einen höheren Impaktfaktor, welcher den Wert der Zeitschrift erhöht. Der Arbeitsplatz des Autors gelangt in das Bewusstsein der wissenschaftlichen Gemeinschaft, welche somit einen besseren Überblick über die bearbeiteten Themen erhält, und es kommt zu keiner Überschneidung der Forschung. Die frei zugänglichen Fachpublikationen gewinnen auch für die Popularisierung der Wissenschaft eine Bedeutung, da sie auch in den Reihen der Laien zu größerer Bekanntheit gelangen.

Einen Artikel in einer wissenschaftlichen Prestigezeitschrift unterzubringen geht weder einfach noch schnell. Mit der steigenden Notwendigkeit seitens der Wissenschaftler, in offenen Zeitschriften zu publizieren, finden sich auch immer mehr Betrüger, so genannte unseriöse Zeitschriften, welche im goldenen Weg des Open Access auf eine Goldquelle gestoßen sind. Sie bieten den Wissenschaftlern die Veröffentlichung in einer offenen Zeitschrift an. Deren einziges Ziel besteht jedoch darin, so viel Geld wie möglich durch APC-Gebühren einzuheben. Deshalb gibt es hier kein klassisches Rezensionsverfahren (peer review), wobei die Fachkompetenz des Artikels von Wissenschaftlern des gegebenen Faches beurteilt wird, und der Artikel erst auf Grundlage ihrer Empfehlung für den Druck in seriösen Zeitschriften angenommen wird. Unseriöse Zeitschriften sind bereit, für Geld alles Mögliche zu publizieren, inklusive Plagiate. Die Qualität des Beitrages spielt keine Rolle und dies ist für die Wissenschaft und auch für die Vertrauenswürdigkeit der übrigen OA-Zeitschriften ein großes Problem. Daher schulen wir vor allem junge Wissenschaftler und Doktoranden darin, diese sogenannten scam journals zu erkennen. Ein anderer Typ der Prädatoren sind die so genannten hijacked 
journals, welche sich unberechtigterweise zum Zweck der Gewinnsteigerung die Identität einer eingeführten Fachzeitschrift aneignen. Auf den ersten Blick kann der Wissenschaftler dies nur schwer erkennen. Zum ersten Mal machte Tim Hill im Jahre 2008 auf dieses Problem aufmerksam; aber in den letzten Jahren mehren sich diese Fälle. Während es im Jahre 2011 etwa 18 unseriöse Zeitschriften gab, wurden im Jahre 2016 bereits 1211 verzeichnet.

Die meisten Prädatoren verwenden ein aggressives Marketing. Sie sprechen in Massen-E-Mails wissenschaftliche Mitarbeiter an Universitäten und wissenschaftlichen Arbeitsstellen an und bieten ihnen die Möglichkeit der Veröffentlichung ohne jedwede Einschränkungen an. Häufig führen sie Namen, welche leicht mit so mancher wissenschaftlichen Prestigezeitschrift verwechselt werden kann, oder führen im Redaktionsrat die Namen renommierter Wissenschaftler ohne deren Kenntnis und Einverständnis auf. Manchmal geben sie einen unberechtigten Impaktfaktor, nicht existente Metriken und Identifikatoren oder falsche Angaben an. Sie bemühen sich sogar, ihre "Seriosität" durch ihre ResearchID und ORCID zu belegen. Diese Identifikatoren sind für natürliche Personen bestimmt und werden von den einzelnen Wissenschaftlern selbst eingerichtet. Daher müssen alle diese Angaben überprüft werden. Außer den auf diese Problematik ausgerichteten Schulungen bieten die Hochschulbibliotheken Hilfe bei der Überprüfung der Zeitschrift an, in welcher die akademischen Mitarbeiter publizieren möchten.

Als nützliches Hilfsmittel zur Enttarnung von unseriösen Zeitschriften findet seit dem Jahre 2010 häufig das Verzeichnis von Jeffrey Beall Verwendung, das er in seinem Blog veröffentlicht hat. Einige Gutachter machten darauf aufmerksam, dass die Regeln zur Zuordnung zu diesem Verzeichnis nicht ganz eindeutig sind, und dass dort so manche Zeitschrift unberechtigterweise aufgeführt werden kann. Als Beispiel wurden Zeitschriften des Verlags Frontiers aufgelistet. Im Januar 2017 wurde der Betrieb ohne nähere Begründung eingestellt und das Verzeichnis gelöscht. Dennoch verschwand es nicht vollständig. Es existiert in seiner Archivform. ${ }^{29}$ Leider wird es nicht aktualisiert und stellt damit ein großes Problem dar, da sich die Prädatoren sehr schnell anpassen können. Der Name der Zeitschrift wird geändert, der Verleger taucht unter einem anderen Namen und an einem anderen Ort auf. Es kann ebenfalls vorkommen, dass aus dem ursprünglich seriösen Titel nach der Übernahme durch einen neuen Verleger ein unseriöser Titel wird.

${ }^{29}$ https://web.archive.org/web/20170112125427/https://scholarlyoa.com/publishers/ 


\subsection{APC und Abonnement}

Im Projekt OpenAPC (Open Data on Article Charges) ${ }^{30}$ verzeichnen die beteiligten Universitäten und wissenschaftlichen Gesellschaften die Angaben über die APC, welche sie bezahlt haben. Der durchschnittliche Preis für einen Artikel in komplett offenen Zeitschriften betrug 1488 EUR und in hybriden Zeitschriften 2488 EUR. Die höchste APC entrichtete eine frei zugängliche Zeitschrift von der Max-Planck-Gesellschaft (7 419 EUR) und eine hybride Zeitschrift der University of Oxford (9 070 EUR). ${ }^{31}$ Im Hinblick auf die seitens zahlreicher Projekte formulierte Bedingung, die Ergebnisse im OA-Modus zu publizieren, steigt die Anzahl der offenen Artikel in hybriden Zeitschriften. Die Abonnenten fordern daher zurecht, dass die Höhe der Gebühr für das Abonnement proportional zu diesem Anstieg gesenkt wird. Den Verlagshäusern gelingt es bislang, sich dagegen zu wehren. Es zeigt sich jedoch, dass es die großen Konsortien sind, welche größere Hoffnungen auf Erfolg bei den Verhandlungen haben. Sehr aktiv und auch erfolgreich sind dabei die Niederländer. ${ }^{32}$ Dem niederländischen Verband der Universitäten gelang es in Zusammenarbeit mit den Bibliotheken und unter starker Unterstützung seitens der Regierung, mit den Verlegern eine Reihe von Zugeständnissen auszuhandeln. ${ }^{33}$

In Deutschland entstand das Projekt DEAL ${ }^{34}$, dessen Ziel darin besteht, günstigere gesamtstaatliche Lizenzverträge mit wichtigen Verlegern wissenschaftlicher Literatur abzuschließen und den Status quo im Verhandlungsprozess gerade im Hinblick auf das zu bezahlende APC zu ändern. Das Projekt wurde von der Allianz der deutschen wissenschaftlichen Organisationen initiiert, die durch die Hochschulrektorenkonferenz (HRK) repräsentiert wird.

Da die APC-Kosten in einer Reihe von Projekten anerkannt werden, interessieren sich auch durch die Wissenschaft finanzierende Stiftungen für deren Höhe. Zum Beispiel gehen die Stiftung Wellcome Trust ${ }^{35}$ und die Stiftung Gates $^{36}$ mittels F1000research den Weg eigener Veröffentlichungssportale mit offenem Rezensionsverfahren (openpeer review). Die mit ihren Projekten

\footnotetext{
${ }^{30}$ https://intact-project.org/openapc/

31 https://github.com/OpenAPC/openapc-de

32 http://www.magazine-on-the-spot.nl/openaccess/eng/index.html

33 http://openaccess.nl/en/in-the-netherlands/publisher-deals\#row

34 https://www.projekt-deal.de/about-deal/

35 https://wellcomeopenresearch.org/

${ }^{36}$ https://gatesopenresearch.org/
} 
zusammenhängenden Arbeiten werden sogleich im Open Access veröffentlicht und mit der Zeit werden ihnen Rezensionsgutachten hinzugefügt, so dass der Leser für ein Rezensionsverfahren vorgesehene Artikel, von Rezensenten empfohlene Artikel, bedingt empfohlene Artikel und auch nicht empfohlene Artikel sehen kann. Jedermann kann sich auch die Beurteilungen, die Reaktionen der Autoren und die Kommentare der angemeldeten Nutzer durchlesen.

\subsection{Institutionelle und politische Unterstützung des Open Access}

Förderagenturen und Regierungsinstitutionen, welche die Wissenschaft finanzieren, neigen immer mehr zur Meinung, dass, wenn die Wissenschaft mit öffentlichen Geldern finanziert wird, deren Ergebnisse der fachlichen Öffentlichkeit frei zugänglich sein sollten. Ein bedeutsamer Schritt war die Initiative der Europäischen Kommission, welche für einige Bereiche des 7. Rahmenprogramms die Verpflichtung für die Projektinvolvierten festgelegt hat, einen offenen Zugang zu den Publikationsergebnissen sicherzustellen. Im Programm Horizont 2020 wird diese Verpflichtung noch erweitert: sie betrifft alle rezensierten wissenschaftlichen Artikel, welche mit den Ergebnissen eines Förderprogramms zusammenhängen, und beinhaltet eine dringende Empfehlung bei den übrigen Typen wissenschaftlicher Publikationen. In den so genannten Pilotprojekten (OA data pilot) besteht die Verpflichtung, im Open Access auch alle Forschungsdaten, inklusive der zugehörigen Metadaten zu veröffentlichen, und einen so genannten Data-Management-Plan zu erstellen. Die Daten können entweder zusammen mit der zugehörigen Publikation in einer OA-Datenzeitschrift (z.B. Open Health Data, Scientific Data) oder in einem Daten-Repositorium (z.B. Zenodo, Fishare, DataDryad) veröffentlicht werden.

Mittels des Projektes OpenAire ${ }^{37}$ wurden die erforderliche Infrastruktur für die Speicherung von Daten und eine gemeinsame Unterstützungsstruktur für die beteiligten Repositorien sichergestellt. Die Orientierung in den Anforderungen der Stiftungen und Förderagenturen, die einen offenen Zugang zu Forschungsergebnissen fordern, wird durch den Dienst SHERPA/Juliet ${ }^{38}$ erleichtert. Die Liste der Zeitschriften, welche in Einklang mit den Anforderungen

37 https://www.openaire.eu/

38 http://v2.sherpa.ac.uk/juliet/ 
der Förderer für das Open Access stehen, kann auf SHERPA/Facts ${ }^{39}$ überprüft werden.

Der offene Zugang zu wissenschaftlichen Informationen wurde zu einem untrennbaren Bestandteil der strategischen Dokumente der Europäischen Union. So führt z.B. die Empfehlung der Kommission vom 17.07.2012 über den Zugang zu wissenschaftlichen Informationen und deren Aufbewahrung (2012/417/EU) an:

(5) Das Ziel der Politik des offenen Zugangs besteht darin, einen kostenfreien Zugang zu rezensierten wissenschaftlichen Publikationen und Angaben aus der Forschung in der frühestmöglichen Phase ihrer Verbreitung zu gewährleisten und eine wiederholte Nutzung der wissenschaftlichen Forschungsergebnisse zu ermöglichen. Diese Politik sollte unter Berücksichtigung der Problematik der Rechte auf geistiges Eigentum vollzogen werden.

(6) Die Politik des offenen Zugangs zu den Ergebnissen der wissenschaftlichen Forschung sollte sich auf jedwede aus öffentlichen Mitteln finanzierte Forschung beziehen. ${ }^{40}$

Im Jahre 2016 gab der Europäische Rat für die Konkurrenzfähigkeit bekannt, dass bis zum Jahre 2020 alle aus öffentlichen Geldern finanzierten wissenschaftlichen Arbeiten frei zugänglich sein sollen. Obwohl viele dieses Ziel und die Förderung des Open Access unterstützen, hängt die praktische Umsetzung fest. Nach Robert Jan Smits, welcher zum Sondergesandten der EU für das Open Access ernannt wurde, wird dies durch die gegenwärtige Bewertung der Wissenschaftler nach der Anzahl der Publikationen in renommierten Zeitschriften mit einem hohen Impaktfaktor verursacht. Daher plant er, eine Empfehlung vorzulegen, welche nicht nur auf den freien Zugang zu wissenschaftlichen Publikationen, sondern auch auf das gesamte System ausgerichtet ist, inklusive der Veränderungen, welche vollzogen werden müssen, damit wir das gesetzte Ziel erreichen können. ${ }^{41}$

\footnotetext{
${ }^{39}$ http://www.sherpa.ac.uk/fact/index.php?la=en

40 http://eur-lex.europa.eu/legal-content/CS/TXT/?uri=CELEX:32012H0417

${ }^{41}$ https://horizon-magazine.eu/article/open-access-scientific-publications-must-becomereality-2020-robert-jan-smits_en.html
} 
Die Max-Planck-Gesellschaft, Helmholtz-Gemeinschaft und der Deutsche Bibliotheksverband gehörten zu den ersten Unterzeichnern der Berliner Erklärung im Jahre 2003 und stehen bis heute an der Spitze der Bewegung Open Access. Die Helmholtz-Gemeinschaft zum Beispiel erklärte, dass bis zum Jahr 2020 $60 \%$ ihrer Publikationen und bis zum Jahr 2050 alle Publikationen im OA zu finden sein werden. Bereits im Jahre 2014 verankerte die Bundesregierung im Autorengesetz Bedingungen, welche die Speicherung wissenschaftlicher Publikationen in die Repositorien erleichtern. Seit dem Jahr 2016 verfügt Deutschland über eine genehmigte offizielle nationale Open Access-Strategie (Open Access-Strategie für Deutschland), welche vom Bundesministerium für Bildung und Forschung herausgegeben worden ist. ${ }^{42}$

Der Zugang zum Open Access in der Tschechischen Republik war bis vor kurzem gänzlich von einzelnen Institutionen abhängig. Treibende Kraft für das Open Access waren vor allem die Universitätsbibliotheken, welche diesen Gedanken nicht nur publik machen, sondern meist auch institutionelle Repositorien einrichten und verwalten. An den meisten Universitäten wird daher durch den Verdienst der Bibliotheken die notwendige Infrastruktur für die Speicherung der Publikationen im Open Access geschaffen. Die Anzahl der tatsächlich offenen wissenschaftlichen Publikationen ist jedoch in den einzelnen Institutionen sehr unterschiedlich. Einige Repositorien ermöglichen ihren Besuchern lediglich den Abruf von Bachelor- und Diplomarbeiten. Erst am 14.06.2017 genehmigte die tschechische Regierung die nationale Strategie des offenen Zugangs zu wissenschaftlichen Informationen für die Jahre 2017-2020.

\subsection{Open Access im Projekt Lernraum}

Die internationale Bewegung Open Access wird vor allem von den akademischen Bibliotheken unterstützt, deshalb wurde deren Unterstützung logischerweise auch in das Projekt Lernraum - Bibliothekarische Informationsplattform einbezogen. Unser Ziel bestand in der Propagierung des Gedankens eines offenen Zugangs zwischen den Nutzern, in der Feststellung, wie der Stand vor allem bei den ausländischen Projektpartnern ist, im Erfahrungsaustausch mit der Propagierung von Open Access zwischen unseren Nutzern, mit der Kommunikation und Unterstützung des Universitätsmanagements und nicht zuletzt mit der Verwaltung des Repositoriums.

${ }_{42}$ https://www.bmbf.de/pub/Open_Access_in_Deutschland.pdf 
Die Leitung der Technischen Universität Chemnitz unterstützt das offene Publizieren ihrer wissenschaftlichen Mitarbeiter bereits seit 1995 mit der Bereitstellung eines institutionellen Repositoriums. Im Jahr 2006 wurde die Open Access-Policy der Institution verabschiedet. Im Jahre 2015 unterzeichnete die Universität die Berliner Erklärung und erweiterte im selben Jahr ihre Politik des freien Zugangs. Sowohl der goldene als auch der grüne Weg des Open Access erfahren Unterstützung.

Die Universitätsbibliothek versteht sich als Motor zur Umsetzung offener Wissenschaft an der Technischen Universität Chemnitz und bietet seit mehreren Jahren Service und Know-How zum Open Access-Publizieren an. ${ }^{43}$ Für die Herausgabe eigener Open Access-Zeitschriften wurde die Software Open Journal System ${ }^{44}$ installiert.

Zur Veröffentlichung von hybriden Büchern wurde 2009 der Universitätsverlag ${ }^{45}$ gegründet.

Das Open Access-Team veranstaltet regelmäßig Seminare sowie Schulungen und führt individuelle Beratungen durch. Schulungsmaterial wird online bereitgestellt. Fast alle Bibliotheksprozesse werden durch Open Source-Software realisiert.

Für die vorbildliche Arbeit im Bereich Open Access erhielt die Bibliothek als dritte Einrichtung den Open Library Badge $2016^{46}$.

Auf den Bibliotheks-Webseiten ${ }^{47}$, im Blog ${ }^{48}$, über Facebook und Twitter gibt es Neuigkeiten zu Open Access.

Die Bibliothek verwaltet den Open Access-Publikationsfonds der Technischen Universität Chemnitz zur Unterstützung des goldenen Weges. Der Fonds wird aus Mitteln der DFG, der Bibliothek und seit 2017 aus einem Autorenanteil gespeist. Die Kriterien zur Nutzung des Fonds sind auf der Webseite der Bibliothek aufgelistet. ${ }^{49}$

\footnotetext{
${ }^{43}$ https://www.tu-chemnitz.de/ub/publizieren/openaccess/\#policy

44 https://www.bibliothek.tu-chemnitz.de/ojs/

45 https://www.tu-chemnitz.de/ub/univerlag/index.html

46 https://badge.openbiblio.eu/

${ }^{47}$ https://www.tu-chemnitz.de/ub/publizieren/openaccess/index.html

48 https://blog.hrz.tu-chemnitz.de/bibo/

${ }^{49}$ https://www.tu-chemnitz.de/ub/publizieren/openaccess/\#finanz
} 
Eine Förderung erfolgt nur wenn der Artikel in einer frei zugänglichen Zeitschrift erscheint, wie sie im Directory of Open Access (DOAJ) enthalten sind. Die APC darf maximal 2000 EUR betragen. Der Artikel darf nicht im Rahmen eines anderen Förderprojektes entstanden sein. Die jährlich entstandenen Publikationskosten erscheinen transparent auf der Bibliothekshomepage und werden an das Projekt Open APC gemeldet ${ }^{50}$.

Alle geförderten Artikel werden auch im Repositorium Monarch-Qucosa gespeichert, welches von der Universitätsbibliothek betreut wird ${ }^{51}$. Die Software wird von der Sächsischen Landesbibliothek - Staats- und Universitätsbibliothek Dresden (SLUB) gehostet. Der Mandant Technische Universität Chemnitz beinhaltet aktuell 3604 Publikationen. Nahezu alle Dokumente sind vollständig verfügbar.

Auch an der Hochschule Zittau/Görlitz wächst die Bedeutung des offenen Publizierens. Einige wissenschaftliche Mitarbeiter publizieren bereits seit längerer Zeit im Open Access und machen es selbst publik. Für andere wird die Problematik vor allem im Zusammenhang mit den Anforderungen der Förderer aktuell. Die Akademiker können ihre Publikationen in das sächsische Repositorium Qucosa ${ }^{52}$ mittels der SLUB speichern.

Die Hochschulbibliothek begann im Rahmen des Projektes ihre Nutzer über die Problematik des offenen Publizierens in Form von Seminaren und Informationsmails zu informieren. Es wurden Webseiten mit Basisinformationen über Open Access angelegt ${ }^{53}$. Die Mühe der Hochschulbibliothek wurde von Erfolg gekrönt: Gegenwärtig wird eine OA-Strategie der Hochschule Zittau/Görlitz ausgearbeitet, welche noch von der Leitung genehmigt werden muss.

Die Westböhmische Universität in Pilsen unterzeichnete die Berliner Erklärung im Jahre 2015, hat aber bislang keine ausformulierte und genehmigte Open Access-Politik. Die Universitätsbibliothek hat sich der Förderung des OA im Jahre 2010 angeschlossen. Sie veranstaltet regelmäßig Seminare zur Problematik des offenen Publizierens, verfügt über eine Webseite zu Open Access $^{54}$, gibt Informationsflyer heraus und bietet individuelle Beratungen an,

\footnotetext{
${ }^{50}$ https://treemaps.intact-project.org/apcdata/tu-chemnitz/

51 http://monarch.qucosa.de/

52 http://www.qucosa.de/

${ }^{53}$ https://hsb.hszg.de/ueber-uns/projekte/lernraum-2016-2018/open-access.html

54 http://knihovna.zcu.cz/en/open-access/OpenAccess.html
} 
am häufigsten zu Fragen zur Qualität von OA-Zeitschriften. Alljährlich beteiligt sie sich an der Open Access Week.

Seit dem Jahre 2012 betreibt die Bibliothek das institutionelle Repositorium Digitale Bibliothe ${ }^{55}$ unter dem System DSpace. Das Repositorium beinhaltet 27483 Dokumente ${ }^{56}$ und ist in drei grundlegende Kollektionen gegliedert: Publikationstätigkeit (Publications) - Artikel, Konferenzbeiträge und weitere Arbeiten von Autoren der Westböhmischen Universität (5 648 Dokumente); Hochschulqualifikationsarbeiten (Thesis) - Bachelor- und Masterarbeiten (14 496 Dokumente) und frei zugängliche Bildungsquellen (Open Resources) - Dokumente, Medien, Kurse, Modelle oder Software, welche zum Unterricht, zur Lehre, zur Bewertung und für Forschungszwecke eingesetzt werden (93 Dokumente). Bis auf einzelne Ausnahmen findet sich alles im Open Access. Die Digitale Bibliothek der Westböhmischen Universität hat eine tschechische und eine englische Sprachschnittstelle. Zu jedem Beitrag und jeder Kollektion kann eine Nutzungsstatistik eingesehen werden.

Während der Projektdauer wurden beinahe 4000 Dokumente eingearbeitet. Die Version DSpace 5 wurde aktualisiert und es wurde ein neues Design der Digitalen Bibliothek erstellt. Die Basisdatei der Funktionen wurde um Ergänzungsdienste und -funktionen erweitert. Die Bibliothek wurde mit anderen universitären Systemen verbunden: mit der Studienagenda STAG, dem Discovery-System Summon und mit der Software zur Registrierung der Publikationen OBD. Die in der Digitalen Bibliothek gespeicherten Daten werden in das nationale Repositorium der grauen Literatur NUŠL ${ }^{57}$, den Aggregator des Repositoriums OpenAire und ins Register des Repositoriums OpenDOAR importiert.

Im Testbetrieb verläuft die Abgabe aller Artikel und Konferenzbeiträge von zwei Arbeitsstellen der Westböhmischen Universität aus. Diese werden in die Digitale Bibliothek importiert und deren Volltexte werden nach den Richtlinien des Verlages in der Datenbank SHERPA/ROMEO veröffentlicht: Für die übrigen Autoren ist die Speicherung der Publikationen in das Repositorium bislang freiwillig. Darüber hinaus nutzt eine Reihe Autoren der Westböhmischen Universität ebenfalls den goldenen Weg. Die APC werden teilweise aus

55 https://dspace.zcu.cz/

56 Stand vom Mai 2018.

57 http://www.nusl.cz/ 
Projekten und zum Teil von der Arbeitsstelle des Autors gedeckt. Die Universität unterhält keinen Unterstützungsfonds.

Die Universitätsbibliothek der Technischen Universität Liberec unterzeichnete die Berliner Deklaration mittels des Verbands der Hochschulbibliotheken in der Tschechischen Republik (Asociace knihoven vysokých škol ČR) im Jahre 2012. Über eine offizielle OA-Politik verfügt die Universität indes bislang nicht. Über die Problematik des offenen Publizierens informiert die Bibliothek in Vorträgen für die Öffentlichkeit und in einmaligen Kursen. Sie fügt dieses Thema auch in den Unterricht ein und bietet auch individuelle Beratungen an. Sie unterstützt Open Access durch Informationsflyer, Plakate, auf Webseiten und auch in sozialen Netzwerken. Im Rahmen der Open Access Week wird als Statement die Farbe Orange in unterschiedlichen Bibliotheksbereichen verwendet.

Die Universitätsbibliothek betreibt ihr Repositorium auf der Plattform DSpace..$^{58}$ Dort sind 22177 Dokumente abgelegt, von denen etwa 70 \% frei zugänglich sind. Den größten Teil bilden Bachelor- und Masterarbeiten sowie Dissertations- und Habilitationsschriften (20 028 Dokumente). Weiterhin sind hier Publikationen von Autoren der Technischen Universität Liberec (421 Dokumente), an der Universität herausgegebene 0A-Zeitschriften (596 Dokumente) und Lehrmaterialien archiviert. Im Mai 2018 entschied die Leitung über die Veröffentlichung von Abschlussarbeiten, und zwar rückwirkend. Bis auf objektiv begründete Ausnahmen werden alle Arbeiten im Open Access zugänglich sein.

Die Metadaten der Publikationen universitärer Autoren aus der Zitatendatenbank WoS und Scopus werden regelmäßig importiert. Zu diesen werden von der Bibliothek die Volltexte gesucht und ihnen hinzugefügt.

Im Verlaufe des Projektes ging die Bibliothek zur Version 6.2 DSpace über, ordnete das Repositorium neu, sortierte und erweiterte die Metadaten in eine maschinell besser lesbare Form. Seit dem Jahr 2009 bis zur Gegenwart digitalisierte und speicherte sie im Repositorium die Universitätszeitschrift ACC JOURNAL. Sie digitalisiert durchgehend alle Forschungs- und Technikberichte der Technischen Universität Liberec.

Das Repositorium wurde mit der Studienagenda STAG verbunden, was einen direkten Import der vollständigen Texte der Abschlussarbeiten ermöglicht.

${ }^{58}$ https://dspace.tul.cz 
Weiter nehmen die Mitarbeiter der Bibliothek an der Überführung von Datenkanälen aus den universitären Systemen in die Evidenz der Ergebnisse von Wissenschaft und Forschung (https://publikace.tul.cz) teil. Dies ermöglicht nach dem Ablauf des Zeitverzugs die Veröffentlichung der Ergebnisse der exzellenten Forschung an der Technischen Universität in Liberec.

\subsection{Die Systeme der Repositorien der Projektpartner}

Die deutschen Partner nutzen das Repositorium Qucosa, welches über das System Fedora von der Sächsischen Landesbibliothek - Staats- und Universitätsbibliothek Dresden (SLUB) entwickelt wurde und verwaltet wird. Deren einzelne Teile können als Repositorium der einzelnen Institutionen dienen. Auf diese Weise wird es von acht Organisationen in Sachsen inklusive der Technischen Universität Chemnitz genutzt. Gleichzeitig können mittels der SLUB auch Autoren anderer Universitäten und Hochschulen ihre Arbeiten dort einstellen. So wird es auch von der Hochschule Zittau/Görlitz genutzt. Man kann entweder in allen eingestellten Dokumenten oder nur in einem konkreten Repositorium suchen.

Der Publikationsserver Qucosa dient zur langfristigen Archivierung von Dokumenten und deren Zugänglichkeit. Alle Arbeiten haben einen dauerhaften Identifikator (urn:nbn) und werden mit Hilfe von strukturierten Metadaten beschrieben. Die Dokumente sind auch in den Katalogen der beteiligten Bibliotheken, der Deutschen Nationalbibliothek und auch mit dem Dienst Google Scholar auffindbar.

Beide tschechischen Partner nutzen das Open-Source-System DSpace, welches sich $43 \%$ der Repositorien in der Welt zunutze machen. Es ist für akademische, gemeinnützige und kommerzielle Organisationen bestimmt, welche ein offenes digitales Repositorium schaffen. Zu seinen Vorzügen zählen die einfache Installation, völlige Anpassungsfähigkeit und auch die Anbindung an die übrigen Systeme. Eine Selbstverständlichkeit sind die verschiedenen Sprachversionen des Systems.

DSpace bewahrt und ermöglicht einen leichten und offenen Zugang zu allen Typen digitalen Inhalts inklusive Texten, Bildern, beweglichen Bildern, Videos, MPEG-Dateien und Datensätzen in verschiedenen Formaten. Den einzelnen Dokumenten ist der dauerhafte Identifikator handle zugeordnet, welcher von Google indexiert wird. 
Die gespeicherten und archivierten Dokumente können nach Kollektionen, Autoren, Titeln, Schlüsselwörtern oder nach der Art des Dokuments durchsucht (browse) oder herausgesucht (search) werden. Das Heraussuchen ist auch durch die Nutzung der Booleschen Operatoren (AND, OR, NOT) möglich. Das System informiert die Nutzer über neu hinzugefügte Posten und ermöglicht die Einstellung verschiedener Zugangsrechte in Bezug auf die Dokumente oder für einzelne Nutzergruppen.

Alle Repositorien der Projektpartner sind über die Webseiten des Projektes des Leadpartners Technische Universität Chemnitz zugänglich. ${ }^{59}$

\subsection{Gemeinsame Veranstaltungen im Rahmen des Projekts}

Im September 2017 fand an der Technischen Universität in Liberec ein gemeinsamer Workshop statt, auf dem sich die Projektpartner über den aktuellen Stand des Open Access an ihren Institutionen informierten. Die Kollegen der Universitätsbibliothek der Technischen Universität Chemnitz machten die Projektpartner zuerst mit der Gesamtsituation in Deutschland und an der Universität bekannt. Als inspirativ erwies sich die Art und Weise der Nutzung des Publikationsfonds unter Beteiligung des Autors. Die Technische Universität Liberec und die Westböhmische Universität in Pilsen stellten auf dem Workshop ihre institutionellen Repositorien vor, welche mit dem System DSpace arbeiten. An allen drei Universitäten wird außer der Möglichkeit, die Arbeit in das Repositorium einzustellen, auch der goldene Weg der freien Veröffentlichung genutzt.

Für die Bibliothek der Hochschule Zittau/Görlitz war dieses Thema relativ neu. An dieser Hochschule gibt es zwar einige Wissenschaftler, welche für ihre Arbeit die offene Publikation nutzen; doch nur wenige von ihnen publizieren im Open Access. Aufgrund dessen, dass die Initiative Open Access vor allem in Hochschulbibliotheken aktuell ist, ist in diesem Bereich der Projektpartner, die Wissenschaftliche Bezirksbibliothek in Liberec, noch nicht aktiv.

Der Oktober ist traditionell die Zeit, da die internationale Aktion Open Access Week stattfindet, bei welcher besonders die akademischen Bibliotheken den Gedanken des freien Publizierens publik machen. Alljährlich wird ein gemeinsames Motto gewählt, unter welchem die Veranstaltungen stattfinden. Das Motto für das Jahr 2017 Open Access. Open in Order to... wurde

${ }^{59}$ https://www.tu-chemnitz.de/ub/projekte-und-sammlungen/projekte//rbip/infoplattform/ 
auch zum Titel des Pilsener Oktoberseminars zu Informationskompetenzen, welches vor allem für junge Wissenschaftler bestimmt war.

Das Programm beinhaltete einen detaillierten Einblick in die Problematik des wissenschaftlichen Publizierens im Modus Open Access, von grundlegenden Informationen bis zu spezialisierten Vorträgen, welche auf unseriöse Zeitschriften oder auf die Analyse der Kosten der tschechischen Universitäten für die Entrichtung der Publikationsgebühren (APC) ausgerichtet waren. Die Seminarteilnehmer wurden mit den größten tschechischen Repositorien und den wichtigsten weltweiten Registern mit Ausrichtung auf Open Access bekannt gemacht. Das Repositorium der Gastgeberuniversität (Digitale Bibliothek des Westböhmischen Universität) wurde gesondert vorgestellt, inklusive Anschluss an die Software. Diese Software dient zur Sammlung von Informationen über die Publikationen der universitären Autoren (OBD). Das Seminar wurde von 43 Teilnehmern, vor allem Doktoranden aus allen fünf in das Projekt involvierten Organisationen besucht. Im Oktober 2018 fand im Rahmen der Open Access Week mit dem Thema Designing Equitable Foundations for Open Knowledge ein dreitägiger Austauschaufenthalt an der Westböhmischen Universität in Pilsen statt. Von jeder der zusammenarbeitenden Bibliothek kamen zwei Mitarbeiter mit Interesse an der Problematik des freien Publizierens. Im Hinblick auf den unterschiedlichen Grad der Erfahrungen der einzelnen Teilnehmer mit dem OA wurde ein Vortrag mit einer kurzen Einführung in die Problematik und ein praktisches Beispiel der Vorgehensweise bei der Überprüfung einer unseriösen Zeitschrift integriert. Die Projektpartner machten sich detailliert mit dem Pilsener Repositorium und dessen Anbindung an weitere universitäre Informationssysteme sowie mit dem Konzept der offenen Ausbildung vertraut. Bei Exkursionen zu spezialisierten Arbeitsstellen der Universitätsbibliothek konnten sie sehen, wie hier das Open Access publik gemacht wird.

\subsection{Ergebnisse und Schlussbewertung des Meilensteins}

Das Thema Open Access gehört ins Projekt Lernraum, da es den Bereich des Zugangs zu Kenntnissen erweitert. Der Meilenstein zielte auf die Vertiefung der Kenntnisse über Open Access innerhalb der Nutzergrupe, aber besonders auf den Erfahrungsaustausch mit den ausländischen Partnern. Die Beispiele der guten Praxis in Deutschland und in der Tschechischen Republik und vor allem an den einzelnen Universitäten stärkten die Methoden- und 
Bildungskompetenzen der Bibliothekare und bereicherten auch die Nutzer der Bibliotheken um neue Erkenntnisse. Besonders interessant war der Vergleich der Unterstützung des $\mathrm{OA}$ vonseiten der Regierung in Deutschland und Tschechien. Eine Bereicherung waren auch die bei jedem Treffen stattfindenden informellen Debatten über die Problematik - mit dem Ziel, die Effizienz der Vorträge zu stärken, zur Implementierung der ins Leben gerufenen Themen einen Beitrag zu leisten, die Selbstreflexion der Teilnehmer im Bezug auf konkrete Anforderungen der Nutzer, der Universitäten und der Förderagenturen zu fördern (z.B. Erstellung der Webseite über das OA), einen Fonds zur Förderung des OA-Publizierens zu gründen und eine universitäre Richtlinie zu schaffen. Die Förderagenturen reagierten auch auf die derzeitigen Bedürfnisse der Teilnehmer (auch im Hinblick auf das unterschiedliche Niveau der Unterstützung des $\mathrm{OA}$ an den einzelnen Universitäten) und auf die Fragen und Probleme, welche nicht ins offizielle Programm aufgenommen waren (z.B. Datenrepositorien, Zusammenarbeit mit Projektzentren). Die gegenseitige Inspiration und die Schöpfung aus den Erfahrungen der anderen halfen den Teilnehmern über ihre Schwächen und Stärken nachzudenken und lieferten Anregungen zur Verbesserung. Im Hinblick auf den wachsenden Einfluss des OA und auf den Bedarf, es beim wissenschaftlichen Publizieren praktisch zu nutzen, war dieser Meilenstein für alle Teilnehmer sehr effizient. Insgesamt bewerten wir den Meilenstein in jeder Hinsicht als sinnvoll. 


\title{
INFORMATIONSKOMPETENZ IM GRENZÜBERGREIFENDEN LERNRAUM
}

Oder: was in einem Informationskompetenzmodul vermittelt werden muss

\author{
Angela Malz \\ Václav Ovčačík
}




\subsection{Projektaktivitäten}

Dass Bibliotheken Schulungen und Führungen für ihre Nutzer anbieten, ist nicht neu. Es gehört zu den grundlegenden Aufgaben von Bibliotheken, ihre Nutzer in die Bibliotheksbenutzung und in die Recherchemöglichkeiten einzuführen. Früher waren es Zettelkataloge, gedruckte Bibliographien und Nachschlagewerke, die den Benutzern nahe gebracht werden mussten. Heute, im Zeitalter der digitalen Bibliothek, sind es Datenbanken, Fachportale und Onlinekataloge, mit denen Bibliotheksbenutzer umgehen. Bibliotheksbenutzer müssen lernen, aus einer Vielzahl von Informationen die heraus zu filtern, die sie für ihre Fragestellung benötigen. Diese Informationen müssen zudem aufbereitet und bewertet werden. Dafür benötigen sie eine Fähigkeit, die als Informationskompetenz bezeichnet wird.

In Vorbereitung des Antrages für das Projekt Lernraum - Bibliothekarische Informationsplattform war schnell klar, dass auch das Thema Vermittlung von Informationskompetenz als ein Meilenstein in das Projekt aufgenommen werden soll.

Im Projektantrag steht dazu:

„Unter Informationskompetenz versteht man die Fähigkeit, Informationen zu finden, aufzubereiten und zu bewerten. Die Bibliotheken streben eine bessere Integration der Informationskompetenz in Lehre, Forschung und Studium an. Ein eigens entwickeltes, didaktisches Informationskompetenzmodul in gedruckter und elektronischer Form soll dazu dienen. Weitere Konzepte sollen im Rahmen des Projektes erörtert werden."

Folgende Aktivitäten waren zur Umsetzung des Meilensteines geplant:

- 09.05.2017 Informationskompetenz-Tag in der Universitätsbibliothek Chemnitz - 25.04.2017 Informationskompetenz-Tag in der Bibliothek Zittau (Webinar) - 26.09.2018 Workshop in der Universitätsbibliothek Chemnitz

Diese Aktivitäten gingen in zwei Richtungen. Einerseits sollten die Nutzer der Bibliothek in Informationskompetenzveranstaltungen mit den Recherchemöglichkeiten der Bibliotheken vertraut gemacht werden und vor allem die Medien vorgestellt bekommen, die im Rahmen des Projektes angeschafft wurden. Diesem Ziel dienten die beiden Informationskompetenz-Tage in 
Chemnitz und Zittau. Nachwuchswissenschaftler und Nachwuchswissenschaftlerinnen sowie Studierende der Partnereinrichtungen nahmen daran teil. In der Universitätsbibliothek Chemnitz bekamen sie einen Einblick in die digitale Bibliothek und erfuhren Interessantes zum Exzellenzcluster MERGE. Der Informationskompetenz-Tag, der in Zittau geplant war, wurde als Webinar durchgeführt. Die beteiligten Bibliotheken stellten vor allem die Medien vor, die im Rahmen des Projektes erworben wurden. Gleichzeitig konnte die Technik eingesetzt werden, die innerhalb des Meilensteines 2 (Technische Ausstattung) erworben wurde. Andererseits beschäftigten sich die Bibliothekare mit dem Thema Informationskompetenz. Das Thema wurde theoretisch aufgearbeitet, Konzepte verglichen und ein Informationskompetenzmodul entwickelt.

Am 23. und 24. Mai 2017 trafen sich Bibliothekare aus allen Projektbibliotheken im Rahmen der Praxiswoche in Liberec und Görlitz. Neben Themen, die die technische Ausstattung des Lernraumes betrafen, tauschten sie ihre Erfahrungen auf dem Gebiet der Vermittlung von Informationskompetenz aus. Schnell wurde deutlich, dass die Bibliotheken sehr unterschiedliche Voraussetzungen mitbringen. Die meisten Erfahrungen mit der Vermittlung von Informationskompetenz haben die Universitätsbibliotheken in Chemnitz und Pilsen. In angeregten Diskussionen wurde der Rahmen für das Informationskompetenzmodul abgesteckt. Welche Ziele sollen erreicht werden, welche

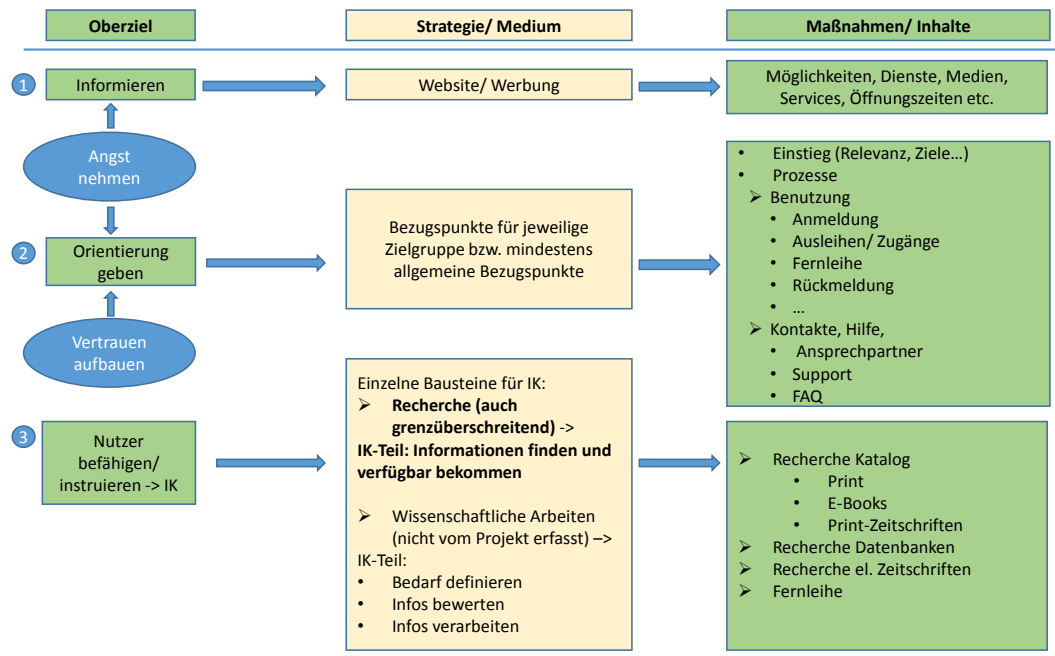

Abbildung 1. Grundlage für das Informationskompetenzmodul. Autor: Falk Maiwald. 
Inhalte sind zu vermitteln, welche Zielgruppen sollen erreicht werden, mit welchen Maßnahmen sollen die Ziele erreicht werden? Als Ergebnis entstand die vorherstehende Grafik, die als Grundlage für alle weiteren Betrachtungen diente.

\subsection{Entwicklung des Informationskompetenzmoduls}

Am 3. August 2017 trafen sich Mitarbeitende aus der Hochschulbibliothek Zittau, der Universitätsbibliothek in Liberec und der Wissenschaftlichen Bibliothek in Liberec, um weiter am Meilenstein Informationskompetenz zu arbeiten. Es entstand der Plan, in Moodle für jede Bibliothek die wichtigsten Informationen zur Bibliotheksbenutzung in Form von FAOs einzustellen. Ein entsprechender Fragenkatalog wurde erarbeitet.

Am 26. September 2017 wurde ein Workshop in der Universitätsbibliothek in Liberec genutzt, um den Stand der Arbeiten vorzustellen. Ob Moodle als Plattform für das Informationskompetenzmodul wirklich die geeignetste Plattform ist, konnte nicht abschließend geklärt werden. Vor allem die deutschen Projektpartner hatten Vorbehalte, weil sie Moodle als Lernplattform nicht nutzen.

Deshalb wurde die Diskussion zum Workshop am 14. Februar 2018 in der Universitätsbibliothek der Westböhmischen Universität in Pilsen fortgeführt. Die Projektpartner einigten sich darauf, dass Moodle als ein weiteres Konzept betrachtet wird (siehe Kapitel 3.4).

Der Leadpartner wurde beauftragt, ein neues Konzept für das „eigens entwickelte, didaktische Informationskompetenzmodul in gedruckter und elektronischer Form" zu entwerfen.

Die Universitätsbibliothek Chemnitz hat bei der Vermittlung von Informationskompetenz einen reichen Erfahrungsschatz. Mit der Einführung der Bachelorund Masterstudiengänge bietet die Universitätsbibliothek Chemnitz seit dem Wintersemester 2006/2007 Informationskompetenzveranstaltungen an, die teilweise in die Curricula integriert sind. Wenig später entwickelte die Universitätsbibliothek Chemnitz eigene E-Learning-Kurse, weil die Bibliotheksnutzer den Wunsch hatten, von der Bibliothek zeit- und ortsunabhängig Informationen zu bekommen. Für diese E-Learning-Kurse wurden Erfahrungen anderer Bibliotheken genutzt, z.B. die der Universitätsbibliothek Konstanz und die aus dem Projekt lotse ${ }^{60}$.

${ }^{60}$ https://www.ulb.uni-muenster.de/ulb-tutor/lotse/ 
Auf der Grundlage der vielen Erfahrungen der Universitätsbibliothek Chemnitz entstand ein Informationskompetenzmodul, das auch für andere Bibliotheken einsetzbar ist und für alle Projektpartner sowohl in deutscher als auch in tschechischer Sprache zur Verfügung steht.

Das Informationskompetenzmodul ist so aufgebaut, dass es für verschiedene Zielgruppen und Fragestellungen benutzbar ist. Je nach Anlass können die einzelnen Module verschieden zusammengesetzt oder auch unabhängig voneinander verwendet werden.

Das Modul kann von allen interessierten Bibliotheken nachgenutzt werden. Die individuellen Inhalte - bezogen auf die einzelne Bibliothek - müssen angepasst werden. Die bibliotheksunabhängigen Informationen können ohne Änderungen verwendet werden. Das Modull61 steht unter der Lizenz CC BY.

Im September 2018 fand der letzte Workshop des Meilensteines Informationskompetenz in der Universitätsbibliothek Chemnitz statt. Dort wurden die Informationskompetenzmodule sowohl auf Deutsch als auch auf Tschechisch in die finale Form gebracht.

\subsection{Aufbau des Informationskompetenzmoduls}

\section{Modul 1 - Universitätsbibliothek \& Katalog}

Folgende Fragen beantworten wir in diesem Modul:

- Wie funktionieren eigentlich wissenschaftliche Bibliotheken?

- Was kann ich alles in Bibliothekskatalogen finden und was nicht?

- Wie finde ich mein Buch in der Bibliothek? Und wie kann ich es dann ausleihen?

- Semesterapparate - was ist das und gibt es das in jeder Bibliothek?

- Wo kann ich weiter suchen, wenn meine Bibliothek ein Buch oder eine Zeitschrift nicht besitzt?

Inhalt:

- Universitätsbibliotheken

- Katalog

- Sucheinstieg

${ }^{61}$ https://www.tu-chemnitz.de/ub/kurse-und-e-learning/elearning/studierende/ikonline.html 
- Hinweise

- Tutorial Katalog

- Ergebnisliste

- Ranking

- Einschränken

- Exemplaranzeige

- Tutorial Exemplaranzeige

- Erweiterte Suche

- RVK

- Beispiele

- Semesterapparate

- Überregional suchen

- KVK

- Abschlusstest

\section{Modul 2 - Vorbereitung der Recherche}

Folgende Fragen beantworten wir in diesem Modul:

- Wie können Sie Ihre Suche strukturiert planen, um Zeit zu sparen?

- Was sind Datenbanken und wie finden Sie die für Sie passenden?

Inhalt:

- Datenbank - Begriff

- Datenbankinfosystem

- Tutorial Datenbankinfosystem

- 5-Schritte-Modell:

- Was suche ich?

- Tutorial Themenanalyse

- Suchbegriffe sammeln

- Was brauche ich?

- Wo suche ich?

- Wie suche ich?

- Sichten und modifizieren

- Abschlusstest 


\section{Modul 3 - Suchtechniken und Datenbankfunktionen}

Folgende Fragen beantworten wir in diesem Modul:

- Welche Suchtechniken Sie einsetzen können, um effizient in Datenbanken zu suchen?

- Welche komfortablen Funktionen Datenbanken darüber hinaus bieten?

Inhalt:

- Suchtechniken

- Suchfelder/Suchbegriffe

- Suchfelder wählen

- Suchbegriffe finden

- Stichwort / Schlagwort

- Tutorial Schlagwörter

- Thesaurus

- Index

- Suchwerkzeuge

- Trunkierung

- Phrasen suchen

- Boolsche Operatoren

- Tutorial Boolsche Operatoren

- Datenbankfunktionen

- Search History

- Alerting

- Hilfefunktionen

- Abschlusstest

\section{Modul 4 - Recherchieren im Internet}

Folgende Fragen beantworten wir in diesem Modul:

- Welche Suchmaschinen und Webseiten für das wissenschaftliche Arbeiten besonders nützlich sind?

- Wie Sie im Internet effektiv nach Informationen, insbesondere wissenschaftlichen Texten, suchen können?

- Welche im Internet gefundenen Dokumente Sie für Ihre wissenschaftliche Arbeit verwenden können? 
Inhalt:

- Informationsquellen im Internet

- Allgemeine Suchmaschinen

- Surface, Deep \& Darknet

- Spezialsuchmaschinen

- Beispiele Spezialsuchmaschinen

- Fachportale

- Wichtige Fachportale

- Fachportale der UB Chemnitz

- Suchtechniken

- Beispiele

- Sichten \& Modifizieren

- Abschlusstest

\section{Modul 5 - Literaturbeschaffung}

Folgende Frage beantworten wir in diesem Modul:

- Wie kommen Sie an Literatur, die Sie bei der Recherche gefunden haben? Inhalt:

- Ausleihen und Bestellen

- Auffinden von Volltexten

- Tutorial Dokument verfügbar

- Fernleihe / Dokumentlieferdienst

- Fernleihe

- Tutorial Fernleih-Bestellung

- Dokumentenlieferdienste

- Anschaffungsvorschlag

- Abschlusstest

\section{Modul 6 - Zitieren und Plagiate}

Folgende Fragen beantworten wir in diesem Modul:

- Was alles beinhaltet Zitieren? 
- Wie Sie zitieren sollten und was man aus wissenschaftlichen Werken übernehmen kann?

- Was ist ein Plagiat?

Inhalt:

- Zitieren

- Zitierregeln

- Direktes/ indirektes Zitieren

- DIN

- Plagiate

- Definition

- Beispiele

- Folgen von Plagiarismus

- Urheberrecht

- Abschlusstest

\subsection{Konzept in Moodle}

Das Modul ${ }^{62}$ Informationskompetenz in der Lernplattform Moodle wurde nach und nach während der gesamten Projektlaufzeit Lernraum - Bibliothekarische Informationsplattform entwickelt. Bei einem der ersten Projekttreffen zu diesem Thema wurde die freie Software Moodle gewählt, das meistgenutzte E-Learning-System in der Tschechischen Republik (im Rahmen der Diskussionen wurde außerdem das in Deutschland genutzte System OPAL - OnlinePlattform für Akademisches Lehren und Lernen in Erwägung gezogen). Im Laufe des Projektes verzichteten zwar die deutschen Partner wegen mangeInder Beliebtheit auf deren Nutzung, nichtsdestotrotz trugen die Kollegen aus Chemnitz und Zittau/Görlitz mit ihren Vorschlägen, Erfahrungen und Ideen zum Modulentwurf und dessen Bildung bei.

Aus dem ersten Treffen gingen einige wichtige Faktoren hervor, die weiterhin in der gesamten Konzeptionsphase des Informationskompetenz-Moduls in Moodle berücksichtigt wurden.

1. Wegen unterschiedlicher Bedürfnisse und Ziele der Partnerbibliotheken muss die Modularchitektur ausreichend offen sein, um den Partnern die Bearbeitung einzelner Teile zu ermöglichen $\rightarrow$ mehrstufige Architektur

62 https://elearning.tul.cz/course/view.php?id=3335 
2. Den größten Nutzen bringt die Konzentration auf Probleme, die die Nutzer alltäglich zu bewältigen haben $\rightarrow$ die Form der häufig gestellten Fragen und Antworten

Die wichtigsten Fragen stellte die Universitätsbibliothek der Technischen Universität in Liberec zusammen. Die Partner konnten danach die Fragen kommentieren und nach eigenem Erwägen bearbeiten. Es entstand so eine Liste individueller Fragen mit Ausrichtung auf konkrete Leistungen der Partnerbibliotheken.

Die primäre Unterteilung erfolgte nach Fragenkategorien. Hier entschieden sich die Partner für eine Aufteilung nach Zielgruppen - Studenten und Akademiker - und fügten eine neue Kategorie Grundleistungen mit allgemeinen Fragen zur Arbeits- und Funktionsweise einer Bibliothek hinzu.

Nach einigen Gesprächen mit den Projektpartnern wurde noch die Ebene der einzelnen Bibliotheken aufgenommen. Der ursprüngliche Entwurf rechnete mit einer größeren Vernetzung der beteiligten Bibliotheken, bei der es für jede Frage eine allgemeine Antwort gegeben hätte (z.B. Beschreibung der Fernleihe) und erst danach hätte der Nutzer eine spezifische Bibliothek gewählt und wäre zu den konkreten Informationen der Einrichtung gelangt. Die Unterteilung der Bibliotheken wurde an die erste Stelle gesetzt, da einerseits die Unterschiede der angebotenen Leistungen zu groß waren und andererseits die Orientierung für die Nutzer vereinfacht wurde. So konnten Fälle vermieden werden, in denen allgemeine Fragen für einen Service entstanden wären, der nur von einem Partner angeboten wird, z.B. Zuweisung von ORCID - oder Fälle, in denen der Nutzer durch eine allgemeine Antwort die angeforderte Information mit Verzögerung erhält. Dazu unterscheiden sich einige Leistungen nicht nur in verschiedenen Staaten, sondern auch in dessen Rahmen (Funktionsweise der Wissenschaftlichen Bezirksbibliothek und der Universitätsbibliotheken).

Das aktuelle System sieht wie folgt aus:

- Die Startseite mit Vorstellung des Projektes und der Auswahl der Partnerbibliothek

- Die Startseite der gewählten Bibliothek mit Verlinkungen auf Web, Katalog etc. (Repositorium, soziale Netzwerke) und Auswahl der Fragenkategorien

- Fragen

- Antworten

Zum Hinzufügen der Inhalte wurde jedem Partner der Zugang zur Plattform 
gewährt. Dazu wurden die Partner bei gemeinsamen Treffen oder direkt in Moodle mit Hauptregeln und Möglichkeiten des Systems bekannt gemacht. Darüber hinaus wurden ein Diskussionsforum, Anregungen, Kommentare sowie Mitteilungen gegründet. Zum Schluss bevorzugten die Partner jedoch die Kommunikation elektronisch per E-Mail oder telefonisch. Diese Foren wurden somit nur für Arbeitsanweisungen im Kurs genutzt. Die Partner setzten unabhängig voneinander die Inhalte in erstellte Sektionen in der entsprechenden Sprachversion ein, wobei eine Vorgehensweise für die anschließende Übersetzung im Vorfeld abgestimmt wurde. In das MoodleSystem der Technischen Universität in Liberec wurde zu diesem Zweck ein Multi-Language-Content-Filter eingefügt, der ein einfaches Einstellen von Tags für einzelne Sprachversionen ermöglicht.

Die Anpassung der eigentlichen Struktur, die Verwaltung von Moodle und die eventuelle Beratung lagen im Aufgabenbereich der Universitätsbibliothek der Technischen Universität in Liberec. Die Projektpartner konnten sich somit mit ihren Anmerkungen oder Fragen direkt an die beauftragten Personen wenden.

Das Modul wurde im Sommer 2018 übersetzt und am Ende des Jahres für Nutzer der Partnerbibliotheken freigeschaltet. 



\title{
ERKENNTNISSE EINER ERFOLGREICHEN ZUSAMMENARBEIT
}

Oder: Tipps für eine erfolgreiche Umsetzung eines grenzïbergreifenden Projektes

\author{
Dana Petrýdesová \\ Blanka Konvalinková
}


Unser gemeinsames Projekt Lernraum - Bibliothekarische Informationsplattform ist beendet. Auf einem mehr als zwei Jahre währenden Weg sind wir so manchem Interessanten und Erfreulichen begegnet; aber es tauchten auch Probleme auf, die wir am Beginn des Projektes nicht erwartet hatten. Die Lösung dieser Probleme war nicht immer einfach. Wir können jedoch sagen, dass uns jede Lösung um neue Erfahrungen bereichert hat, welche wir in Zukunft bei der Umsetzung weiterer Projekte nutzen können.

Wir haben versucht, unsere persönliche Erfahrung in einigen Punkten zusammenzufassen, und wollen Ihnen damit ein paar Tipps geben, wie man erfolgreich ein grenzübergreifendes Projekt angeht.

\section{Tipp 1: Gründliche Planung}

Haben Sie interessierte Projektpartner und ein vorbereitetes Projektkonzept im Kopf? Vergessen Sie die gründliche Planung nicht. Es ist unerlässlich, dass die Projektaktivitäten von allen Projektpartnern unterstützt werden. Vergewissern Sie sich, dass alle Partner alle Zusammenhänge verstanden haben, wissen, was diese mit sich bringen und mit innen einverstanden sind. Es ist ideal, ein Protokoll vom Treffen anzufertigen, mit dem sich die Partner vertraut machen und mit dessen Fassung sie einverstanden sind. Sie können somit einem Missverständnis im Verlauf des Projektes vorbeugen.

Eine weitere wichtige Aufgabe besteht in der Zusammenstellung des Budgets. Gewiss wird es Ihnen kaum gelingen, ein genaues Budget für die gesamte Dauer des Projektes zusammenzustellen; aber durch eine gründliche Vorbereitung und mit Hilfe einer Marktforschung können Sie sich der optimalen Variante zumindest annähern. Die tschechischen Partner müssen auch das Kursrisiko in Betracht ziehen, wenn ein Absinken des Kurses einen größeren finanziellen Aufwand verursachen kann. Der Partner zahlt dann aus seinem eigenen Budget drauf. Kursschwankungen lassen sich nicht vorhersagen; aber es ist notwendig, bei der Vorbereitung des Budgets damit zu rechnen.

\section{Tipp 2: Publizität}

Die Pflichtpublizität ist eine der wichtigsten Voraussetzungen eines erfolgreichen Projektes, bzw. ohne eine richtige Publizität kommen kein Projektauftritt und kein Informationsmaterial aus und man soll diese nicht unterschätzen. 
Versehen Sie sämtliche Projektauftritte mit dem Logo der Europäischen Union in der richtigen Größe und zugleich mit dem Hinweis auf den Europäischen Fonds für regionale Entwicklung. Empfohlen wird auch die Verwendung des Programmlogos. Ideal ist die wortwörtliche Einführung, dass dem Projekt finanzielle Unterstützung vonseiten der Europäischen Union zuteilwird.

Die Tätigkeiten im Bereich der Publizität werden von allen Projektpartnern ausgeführt, welche sie folgendermaßen bezeichnen sollten:

1. Physische Auftritte des Projektes (z.B. erworbene Ausstattung)

2. Gedruckte Materialien (z.B. Publikationen)

3. Sämtliche Werbemittel

4. Projektwebseiten

5. Räume, in denen Schulungen oder Workshops stattfinden (z.B. Platzierung von Flaggen und Logos in die Präsentationen oder ins Roll-Up usw.), zugleich müssen durch die verpflichtende Publizität die Präsenzlisten oder die Einladungen zu Veranstaltungen gekennzeichnet werden

6. Räume im Gebäude, in denen der Partner wirkt (z.B. Roll-Up)

Im Verlauf des Projektes empfiehlt es sich, in den Medien über das Projekt zu informieren, seien es die traditionellen Medien (Presse, Rundfunk, Regionalfernsehen) oder die elektronischen (Online-Periodika, soziale Netzwerke), gegebenenfalls auch bei weiteren Veranstaltungen, an denen Sie teilnehmen (z.B. Konferenzen, Messen u. ä.).

Die erforderlichen Informationen für eine richtige Publizität finden Sie in den methodischen Unterlagen im Portal des Programms (www.sn-cz2020.eu). Wir empfehlen Ihnen, diese sorgfältig zu studieren und durchgehend im Auge zu behalten; denn von Zeit zu Zeit erfolgen Aktualisierungen.

\section{Tipp 3: Kommunikation mit dem Kontrollorgan}

Aus eigener Erfahrung empfehlen wir allen Antragsstellern, die eine Förderung beantragen, rechtzeitig und in ausreichendem Maße mit dem Kontrollorgan Ihres Landes (CRR, SAB) in Kontakt zu treten. Die Mitarbeiter sind bereit, bei allen Problemen beratend zu helfen, denen Sie im Verlauf des Projektes begegnen können. Es ist günstiger, das Problem zunächst mit dem Kontrolleur zu besprechen und sich mit ihm zusammen auf die Vorgehensweise und eine Lösungsmöglichkeit zu verständigen. Wenn das Problem erst im Anschluss 
gelöst wird, kann es vorkommen, dass einige Fehler nicht mehr behoben werden können und Sie einen finanziellen Verlust erleiden oder einen Teil der Förderung zurückerstatten müssen.

\section{Tipp 4: Sharing}

Das Teilen von Informationen und Materialien kann allen Projektpartnern das „Projektleben“ beträchtlich erleichtern. Für die Anforderungen unseres Projektes entstand auf Anregung der Hochschulbibliothek Zittau/Görlitz eine Plattform, auf der alle wichtigen Materialien und Informationen (Fotodokumentationen, Anwesenheitslisten, organisatorische Informationen u.ä.) gespeichert wurden. Alle Partner haben zu dieser Plattform Zugang und können auf elegante Weise alle gespeicherten Materialien verwenden. Bei der Erstellung von Projektfortschrittsberichten, Presseberichten oder anderen Informationsmaterialien genügt die Anmeldung auf der Plattform, von der alle erforderlichen Dokumente heruntergeladen werden können.

\section{Tipp 5: Gemeinsame Problemlösung}

Ein Projekt, das Fortschritte aufweisen soll, muss in gemeinsamer Arbeit gelöst werden. Die Partner sollten die Aktivitäten gemeinsam planen und umsetzen und gemeinsam Probleme lösen. Daher ist es gewiss angebracht, eine angemessene Anzahl persönlicher Treffen, Workshops oder Seminare einzuplanen. In unserem Projekt haben wir uns sehr aktiv getroffen, auch auf virtuellem Wege. Wir haben regelmäßig einmal pro Monat im Rahmen eines virtuellen Konferenzraums diskutiert. Dieser war zu diesem Zweck vom Leadpartner Technische Universität Chemnitz eingerichtet worden. Einige Partner nutzten zu diesem Zwecke technische Ausstattung, welche sie aus finanziellen Projektmitteln beschafft hatten; andere hingegen nutzten nur eigene technische Mittel. Die eigentliche Vorbereitung der Technik und die Installation waren keineswegs kompliziert und alles ließ sich mit minimalem Aufwand bewältigen. Es war stets eine hervorragende Gelegenheit, problematische Fragen aktiv zu diskutieren und sich über die weitere Entwicklung des Projektes zu verständigen. Dadurch wurde die Kommunikation zwischen den Partnern und die Möglichkeit gesteigert, sich über aktuelle Neuigkeiten, Probleme und deren Lösung auszutauschen, ohne dabei die Anzahl der persönlichen Treffen erhöhen zu müssen. Die virtuelle Konferenz ersparte uns eine Menge Zeit. 


\section{Tipp 6: Schwerpunkt Personal}

Eine große Bereicherung für unser Projekt stellte die Mitwirkung der Muttersprachlerinnen in den Projektteams bzw. der tschechisch sprechenden Mitarbeiterinnen bei den deutschen Partnern dar. Dies erleichterte und beschleunigte die Kommunikation unter den Partnern. Wir müssen selbstkritisch zugeben, dass die tschechischen Partner bei der Planung des Personals diesem Faktor nicht allzu viel Aufmerksamkeit widmeten; diese Tatsache aber bei jeder Gelegenheit lobten, sei es bei der Kommunikation per E-Mail, im virtuellen Umfeld oder bei persönlichen Treffen. Wir danken für diesen guten Einfall. Gewiss ist dies ein interessanter Tipp für jedwedes weitere Projekt.

Es sollte auch eine Selbstverständlichkeit sein, dass die Mitarbeiter, welche sich am Projekt beteiligen, verantwortungsvoll, flexibel und fähig sind, Probleme operativ anzugehen. Nützlich ist zumindest auch eine Orientierungskenntnis des Umfeldes der grenzübergreifenden Projekte und der Prinzipien ihrer Administration. Aber es ist keine unerlässliche Bedingung; alles kann man schnell lernen.

\section{Tipp 7: Keine Angst!}

Ein tschechisches Sprichwort sagt: „Wer sich fürchtet, darf nicht in den Wald." Dieses Sprichwort charakterisiert auch unseren letzten Tipp für ein erfolgreiches Projekt. Die Umsetzung grenzübergreifender Projekte kann so manche Probleme enthalten, aber durch die gemeinsame Arbeit der Partner lässt sich alles lösen. Der Nutzen, welcher durch die Umsetzung des Projektes erlangt werden kann, überwiegt sonstige negative Erfahrungen. Mitunter kann sich das, was zunächst nicht realisierbar oder organisatorisch sehr anspruchsvoll erscheint, in eine interessante Aktivität umwandeln, welche langfristig nach Beendigung des Projektes nützlich sein kann. Grenzübergreifende Projekte bieten nicht nur eine Möglichkeit, mit Partnern an fachlichen Themen zu arbeiten, sondern auch, sich gegenseitig mit neuen Erfahrungen zu bereichern und ebenfalls das zu erkennen, was in beiden Ländern unterschiedlich ist und inspirierend sein kann. Außerdem können häufige Arbeitstreffen der Partner in eine schöne gegenseitige Freundschaft münden, worin ein weiterer nicht zu verachtender Beitrag des Projektes besteht. Wir drücken Ihnen die Daumen! 



\section{FRAGEN AN 5 PROJEKTPARTNER}




\section{Angela Malz}

Universitätsbibliothek der Technischen Universität Chemnitz

\section{Weshalb haben Sie sich zu der Teilnahme an diesem Projekt entschieden?}

Die Sächsisch-Tschechische Zusammenarbeit hat an der Technischen Universität Chemnitz eine lange Tradition und ist eng mit dem leider viel zu früh verstorbenen Herrn Prof. Dr. Peter Jurczek verbunden, der diese Zusammenarbeit initiiert hat.

Im Jahr 2003 begann das erste Projekt, das Sächsisch-Tschechische Hochschulzentrum (STHZ). Innerhalb dieses Projektes bekam die Universitätsbibliothek Chemnitz Mittel zugewiesen, um eine Sächsisch-Tschechische Fachbibliothek (STFB) aufzubauen. Mit den Beständen sollte die grenzüberschreitende wissenschaftliche Zusammenarbeit zwischen Tschechien und Sachsen gefördert werden.

Die Universitätsbibliothek Chemnitz beschaffte Bücher in deutscher und tschechischer Sprache, die die deutsch-tschechische Zusammenarbeit zum Inhalt hatten. Fachbücher zu Wirtschaftsthemen, zur Geschichte von Sachsen und Böhmen oder zur EU-Osterweiterung kamen dazu. Es wurden auch belletristische Werke, Sprach- und Tourismusführer, Kartenmaterial, DVDs und Hörbücher in den Bestand aufgenommen.

2006 lief das Projekt STHZ aus. Im Nachfolgeprojekt Sächsisch-Tschechisches Hochschulkolleg standen wieder Mittel für die Fachbibliothek zur Verfügung.

Im Sommer 2009 begann die Sächsisch-Tschechische Hochschulinitiative. Erstmals spielten die Bibliotheken der teilnehmenden Universitäten im Projektantrag eine größere Rolle. Betont wurden neben dem Ausbau der SächsischTschechischen Fachbibliothek der grenzüberschreitende Leihverkehr und die verstärkte Kooperation der Bibliotheken.

Die Partner der Universitätsbibliothek Chemnitz waren damals die Bibliothek der Universität Jan Evangelista Purkyně (UJEP) in Ústí nad Labem und die Universitätsbibliothek der Westböhmischen Universität in Pilsen. 
Am Ende des Projektes stand fest, dass die Bibliotheken weiter zusammenarbeiten wollen und andere Bibliotheken des sächsisch-tschechischen Grenzraumes einbeziehen möchten.

Vom 01.12.2013 bis 30.11.2014 wurde das Projekt Sächsisch-Tschechisches Bibliotheksnetzwerk - Informationen grenzenlos durchgeführt. Projektpartner waren die Universitätsbibliothek der Technischen Universität Chemnitz, die Hochschulbibliothek Zittau/Görlitz, die Universitätsbibliothek der Westböhmischen Universität in Pilsen und die Wissenschaftliche Bezirksbibliothek in Liberec. Ziel des Projektes war der Aufbau eines nachhaltigen und effizienten Bibliotheksnetzwerkes zwischen Sachsen und Tschechien.

Die in diesem Projekt gesammelten positiven Erfahrungen und die persönlichen Kontakte führten im Jahr 2015 zu dem Entschluss, innerhalb des Kooperationsprogramms zur Förderung der grenzübergreifenden Zusammenarbeit zwischen dem Freistaat Sachsen und der Tschechischen Republik 2014-2020 ein neues Projekt zu beantragen - das Projekt Lernraum - Bibliothekarische Informationsplattform.

\section{Wie hat Ihre Bibliothek aus der Teilnahme profitiert?}

Wir haben materiell profitiert. Um nur zwei Beispiele zu nennen: Die E-Books von Taylor \& Francis hätten wir ohne die Projektmittel nicht nutzen können. Auch das Whiteboard wäre aus dem eigenen Haushalt nicht finanzierbar gewesen.

Wertvoller als diese materiellen Werte sind die menschlichen. Wir haben fachlich hochqualifizierte Bibliothekarinnen und Bibliothekare kennengelernt, die uns immer mit großer Herzlichkeit empfangen haben. Wir haben viel über die tschechische Bibliothekslandschaft erfahren und gemerkt, dass wir vor den gleichen Herausforderungen der digitalen Gesellschaft stehen. Die Herausforderungen sind nur grenzübergreifend zu meistern.

\section{Welche Projektergebnisse werden Sie in der Bibliothek auch nach Beendigung des Projektes weiterhin nutzen?}

Es gibt kein Projektergebnis, was nicht nach Projektende noch nützlich wäre. Wir können elektronische Ressourcen weiternutzen, haben zeitgemäße Technik, zweisprachige Kataloge, Webseiten und ein nachnutzbares E-Learning-Modul zur 
Vermittlung der Informationskompetenz. Die entstandene Informationsplattform wird weiter gepflegt und so die Verzahnung der Bibliotheken beibehalten.

\section{Welche Erfahrungen nehmen Sie aus dem Projekt mit? Wollten Sie etwas besser machen oder verbessern?}

Wie schon erwähnt, habe ich gelernt, dass wir alle vor den gleichen Herausforderungen der digitalen Gesellschaft stehen und uns internationale Erfahrungen in der täglichen Arbeit sehr nützen. Diese Erfahrungen müssen nicht immer nur aus der westlichen Welt kommen, denn auch bei den Nachbarn im Osten von Deutschland gibt es jede Menge kluge Ideen.

\section{Nehmen Sie Potenzial für eine Erweiterung um ein weiteres Projekt wahr?}

Auf jeden Fall. Wir benötigen zwar erstmal eine kleine Projektpause, weil uns der Umzug in unser neues Gebäude im Jahr 2019 sehr beschäftigen wird. Im neuen Haus werden uns bestimmt neue Ideen für neue Projekte einfallen. Ich kann mir vorstellen, dass das Thema Open Science viel Potential beinhaltet, aber auch die grenzübergreifende Nutzung elektronischer Medien noch lange nicht ausgereizt ist. 


\section{Falk Maiwald / Ralf Schwarzbach}

Hochschulbibliothek der Hochschule Zittau/Görlitz

\section{Weshalb haben Sie sich zu der Teilnahme an diesem Projekt entschieden?}

Für die Teilnahme an dem Projekt hatten wir mehrere Motive und Ziele. Aufgrund der prädestinierten Lage im Dreiländereck und im Rahmen der damit korrespondierenden Internationalisierungsstrategie unserer Hochschule hat auch die Hochschulbibliothek Zittau/Görlitz (HSB) einen Auftrag zur Erfüllung der Vision einer vernetzten Wissenschaftslandschaft in der Euroregion Neiße. Im vorangegangenen Projekt hatten wir bereits gute Ergebnisse in der grenzübergreifenden Kooperation mit anderen wissenschaftlichen Bibliotheken erzielt und wichtige Erfahrungen gesammelt. Dabei wurden nützliche Impulse zur weiteren Modernisierung und Internationalisierung der Bibliotheken im Interesse ihrer Nutzer abgeleitet.

Mit dem neuen Projekt sahen wir die Chance, gemeinsam mit unseren Partnern elektronische Medien konsortial beschaffen und grenzübergreifend nutzen zu können. Mit Blick auf die veränderten Lernraum-Anforderungen an die Bibliotheken reizte uns auch die Möglichkeit einer maßgeblichen Aufrüstung unserer medientechnischen Infrastrukturen. Und natürlich waren wir auf den Wissenstransfer aus den Universitätsbibliotheken gespannt.

Als weiteres Motiv für eine Projektteilnahme ist der Ausbau des Images der HSB nach innen und außen zu erwähnen. Wir sahen die Chance, unser Bibliotheksteam durch die Projektarbeit und die Untersuchung neuer Themen und Konzepte für eine strategische Weiterentwicklung der Bibliothek zu motivieren und so auf die Hochschule wirksam auszustrahlen. Mit dem Projekt wollten wir aber auch regionale und auch sachsenweite Aufmerksamkeit erlangen, zumal Forschungsprojekte an Fachhochschulbibliotheken eher selten sind.

Von Vornherein war uns wichtig, klare Zielsetzungen im Projekt zu verfolgen und das Projekt maßgeblich mitzugestalten, um diszipliniert konkreten Nutzen für uns und alle Projektpartner zu erreichen. 


\section{Wie hat Ihre Bibliothek aus der Teilnahme profitiert?}

Ein zentraler Gewinn für unsere Bibliothek war selbstverständlich die konsortiale Anschaffung der E-Books von Taylor \& Francis, der Zugang zu E-Zeitschriften-Datenbanken von Taylor \& Francis und Vogue und die aktivierte grenzübergreifende Fernleihe, sowie die Beschaffung der Medientechnik. Damit einher geht das übermittelte bzw. entwickelte Know-How über Medien und Bestände sowie Datenbanken der anderen Projektpartner bzw. über die Nutzung der Medientechnik für Webkonferenzen und die virtuelle Gruppenarbeit. Auch haben wir wichtiges Wissen zum Open Access-Publizieren erlangt, weshalb wir das Thema in der hochschulinternen Diskussion präsenter machen und die Entwicklung einer Open Access-Strategie an unserer Hochschule mit befördern konnten.

Als einen wichtigen Profit sehen wir das nähere Zusammenrücken mit allen Projektpartnern und den Wissens- und Erfahrungszuwachs an. Wir kennen uns nun (noch) besser, haben eine bessere Vorstellung bekommen, wie unterschiedliche bibliothekarische Angelegenheiten und Situationen in Tschechien und Deutschland funktionieren und haben auch die Gründe dafür erkannt. In diesem Bereich der Informationskompetenz-Vermittlung konnten wir die Erfahrung und sogar spezifische Inhalte vom Leadpartner nutzen.

Nicht unerwähnt bleiben sollen die kulturell-kulinarischen Streifzüge in Pilsen und Liberec, bei denen die (inter)kulturelle gegenseitige Inspiration alle Partnerbibliotheken nähergebracht hat. Als Organisation profitierte unsere Bibliothek aus der Kollektiverfahrung: die Bibliotheks-Mitarbeiter konnten gemeinsame Zeit mit anderen Kollegen verbringen und sich über ihr Wissen austauschen. Jeder konnte aber auch für sich Einzelerfahrungen machen und neue Anregungen für die Arbeit und für sich sammeln.

Im Projekt konnten wir auch den öffentlichen regionalen Bibliotheken die Leistungsfähigkeit unserer Kooperation demonstrieren, die einen Eindruck bekommen konnten, was wir gemeinsam mit den Projektpartnern erarbeitet haben. Auch im Verbund der sächsischen Hochschulbibliotheken wurde das Projekt anerkennend und als wegweisend wahrgenommen. Unser diesbezügliches Ziel, unser Image durch das Projekt intern und extern weiter zu verbessern, sehen wir als erfüllt an. 


\section{Welche Projektergebnisse werden Sie in der Bibliothek auch nach Beendigung des Projektes weiterhin nutzen?}

Nach dem Projektende werden selbstverständlich die angeschafften Medien genutzt; eine Fortführung der grenzüberschreitenden Fernleihe werden wir anstreben. Die Nutzung der Medientechnik wird zielgerichtet ausgebaut. Dank der Informationsplattform können sich Nutzer der anderen Bibliotheken über die Bestände und Services informieren. Entsprechende Werbemaßnahmen und Mundpropaganda werden dies unterstützen.

Die Erfahrungen, wie man konferenzähnliche Beratungen - auch länderübergreifend - vor Ort oder sogar virtuell plant und durchführt, können wir selbst nutzen oder damit anderen Akteuren unserer Hochschule beratend zur Seite stehen.

Die partnerschaftliche Verbundenheit mit den Projektpartnern bleibt auch unbedingt bestehen, sodass wir auch in Zukunft aufeinander zugehen, uns treffen und beraten können.

Die Euroregion Neiße wird als Wissenschaftsstandort weiter gestärkt und es kommt weiterhin zum kulturellen Austausch, den wir bereits seit längerem, insbesondere mit der Wissenschaftlichen Bezirksbibliothek in Liberec, projektunabhängig pflegen.

\section{Welche Erfahrungen nehmen Sie aus dem Projekt mit? Wollten Sie etwas besser machen oder verbessern?}

Im Projekt sammelten wir Erfahrungen über die Projektpartner, ihre Arbeitsweisen, Bedingungen, die bei innen anders sind (besonders die Unterschiede zwischen Deutschland und Tschechien). Wir haben auch die Erfahrung gemacht, dass wir routiniert im Stande sind, auf hohem Niveau Konferenzen abzuhalten, wobei alles gut organisiert war und meistens reibungslos verlief. Wir sind nun in der Lage, neue Dinge, die in den Partnerbibliotheken realisiert sind, aufzugreifen und ggf. selbst zu etablieren - die neue Technik, die Vernetzung durch die Fernleihe - und sie im Verbund nutzbar zu machen.

Die Projektleitung der HSB Zittau/Görlitz empfand es als wertvoll und für die Erreichung der Projektziele maßgeblich, als zentrale Projektmitarbeiterin für Planung, Überwachung, Koordination und Durchführung eine tschechische 
Muttersprachlerin mit sehr guten Deutschkenntnissen gewonnen zu haben.

In den regelmäßig abgehaltenen Webkonferenzen erfuhren wir, dass die virtuellen Webkonferenzen viel Zeit sparen und eine gute Abstimmungsgrundlage darstellen. Natürlich sind aber auch die persönlichen Treffen und die persönlichen Kontakte vor Ort wichtig. Insgesamt konnten wir auf beide Weisen den Projektfortschritt gewährleisten. In den Webkonferenzen konnten wir reichhaltige Diskussionen führen und mussten uns nicht nur auf (reiseaufwandsbedingt) seltenere Vorort-Begegnungen oder eine Ping-PongMailkommunikation verlassen.

Als effektiv hat sich die Zuweisung der Verantwortlichkeiten für die einzelnen Meilensteine an die einzelnen Partner erwiesen, wodurch die Projektergebnisse gesichert werden konnten.

\section{Nehmen Sie Potenzial für eine Erweiterung um ein weiteres Projekt wahr?}

Das Fortführen des Open Access-Gedankens und die weitere Optimierung des Informationskompetenz-Prozesses ist eine weitere Bearbeitung durch die Projektpartner sicher wert. 


\section{Miloslava Faitová / Magda Šrajbová}

Universitätsbibliothek der Westböhmischen Universität in Pilsen

\section{Weshalb haben Sie sich zu der Teilnahme an diesem Projekt entschieden?}

Einen grundsätzlichen Einfluss auf die Entscheidung für eine Beteiligung am Projekt hatte meine allseits gute Erfahrung mit der Umsetzung vorangegangener Projekte, welche aus dem Europäischen Fonds für regionale Entwicklung im Programm Ziel 3 gefördert wurden. Dabei handelt es sich konkret um die Projekte Sächsisch-Tschechische Hochschulinitiative (20092012), und Sächsisch-Tschechisches Bibliotheksnetzwerk - Informationen grenzenlos (2013-2014).

Durch das neue Projekt wollte ich an die erfolgreiche und beiderseits sehr gut bewertete Zusammenarbeit mit den Partnerbibliotheken aus den vorangegangenen Projekten anknüpfen, also mit den Bibliotheken in den grenznahen Gebieten Böhmens und Sachsens. Ich betrachtete es als bereichernd und effektiv, im Erfahrungsaustausch in den bewährten Formaten von Seminaren, Workshops und Studienaufenthalten in langfristiger Perspektive fortzufahren und auf diese Weise die Aktivitäten der einzelnen Bibliotheken weiterzuentwickeln.

Als wichtig für die Stärkung der Dienste der Universitätsbibliothek der Westböhmischen Universität in Pilsen empfand ich die Gelegenheit zur Erweiterung des Bibliotheksbestandes der elektronischen Medien, deren Beschaffung aus den Mitteln des Projektes finanziert werden konnte.

Zur Entscheidung für die Teilnahme am Projekt trugen auch meine vorhergehenden Kenntnisse und die Erfahrung mit den Bedingungen für die Umsetzung und die Administration eines Projektes dieses Typs bei.

\section{Wie hat Ihre Bibliothek von der Teilnahme am Projekt profitiert?}

Ein wichtiger Gewinn aus dem Projekt war vor allem die Konsortialbeschaffung elektronischer Medien und von E-Book-Titeln des Prestigeverlages Taylor 
\& Francis. Die Universitätsbibliothek der Westböhmischen Universität erhielt dank der Umsetzung des Projektes in den Jahren 2017-2018 Zugang zu zwei Kollektionen der Datenbank für wissenschaftliche Zeitschriften (Social Science \& Humanities Library, Science \& Technology Library) und in einem Zeitraum von 1,5 Jahren Zugang zu 5000 E-Books mit der Option der dauerhaften Anschaffung der meist verwendeten Titel.

Es wurde die Handbibliothek für deutsche Literatur, der sogenannte Semesterapparat, in der pädagogischen Bibliothek der Westböhmischen Universität erweitert, welcher den Germanistik-Studierenden als Quelle für die Arbeit in den Seminaren und zur Vorbereitung auf die Staatsexamina dient.

Es wurde die erforderliche technische Ausstattung für die Bibliothek angeschafft, Notebooks und ein Beamer.

Den Studierenden und den akademischen Mitarbeitern der Westböhmischen Universität in Pilsen wurde eine einzigartige Möglichkeit der kostenlosen internationalen Fernleihe aus den deutschen Bibliotheken der Technischen Universität Chemnitz und der Hochschule Zittau/Görlitz angeboten und vermittelt.

Im Rahmen von Workshops und Seminaren wurden die Kenntnisse und Erfahrungen der tschechischen und deutschen Partnerbibliotheken erfolgreich mitgeteilt und diskutiert, und zwar besonders im Bereich der Informationskompetenz, der technischen Ausstattung der Bibliotheken, der elektronischen Medien, der Unterstützung von Open Access zu den publizierten Ergebnissen und auch im Bereich weiterer aktueller Themen.

Bei Studienaufenthalten machten sich die Angestellten der Bibliothek ausführlicher mit der Tätigkeit der kooperierenden Bibliotheken vertraut und tauschten in intensivem Kontakt ihre Erfahrungen aus. So hatten sie die Möglichkeit eines tieferen Einblicks in den Betrieb der Partnerinstitutionen.

Nicht zuletzt ermöglichte die Umsetzung des Projektes die Veranstaltung eines Seminars für Doktoranden der Universitäten zur aktuellen Problematik des Open Access. 


\section{Welche Projektergebnisse werden Sie in der Bibliothek auch nach Beendigung des Projektes weiterhin nutzen?}

Ergebnisse von dauerhaftem Wert sind:

- Zugang zum Bestand der E-Books des Verlags Taylor \& Francis

- Fortsetzung der internationalen Fernleihe mit der Technischen Universität Chemnitz und der Hochschule Zittau/Görlitz

- Nutzung der technischen Ausstattung für die Bibliothekstätigkeit

- bestehende und vertiefte Kontakte mit den Partnerbibliotheken

\section{Welche Erfahrungen nehmen Sie aus dem Projekt mit? Wollten Sie etwas besser machen oder verbessern?}

Die Erfahrungen mit der Umsetzung des Projektes sind positiv und eine Motivation für die weitere Arbeit. Vor allem möchte ich die ausgezeichnete, freundschaftliche, offene, effektive und operative Zusammenarbeit mit den Partneruniversitäten betonen und die tatsächliche gegenseitige Zusammenarbeit unterstreichen.

Es gelang uns festzustellen, wie es in den einzelnen Bibliotheken funktioniert, uns gegenseitig das Angehen verschiedener aktueller Herausforderungen vorzustellen, uns gegenseitig Beispiele guter Praxis und erworbene Kenntnisse mitzuteilen und die Erfahrungen in die Qualität der von uns angebotenen Dienstleistungen zu projizieren.

Nach der guten Erfahrung mit Videokonferenzen und Webseminaren möchten wir diese Aktivitäten weiter ausbauen und daher die Anschaffung einer hochwertigen Ausstattung zur Umsetzung erwägen.

Auch bei wiederholter Erfahrung war die administrative Seite des Projektes relativ kompliziert, auch wenn ich eine gute Kommunikation mit dem Zentrum für regionale Entwicklung feststellen konnte.

\section{Nehmen Sie Potenzial für eine Erweiterung um ein weiteres Projekt wahr?}

Wir erwägen kein weiteres Projekt im Rahmen des Kooperationsprogrammes zur Förderung der grenzüberschreitenden Zusammenarbeit zwischen dem 
Freistaat Sachsen und der Tschechischen Republik 2014-2020. Wir könnten es auch gar nicht mehr umsetzen.

Sollte in den nächsten Jahren ein neues Programm bekannt gegeben werden, aus deren Projektaktivitäten wir Unterstützung erfahren können, werden wir es in Erwägung ziehen, uns anzuschließen.

Der Bereich des Bibliothekswesens muss mit den Trends in der Bildung und dem Informationsstand der Gesellschaft mithalten können. Es wird weiterhin vonnöten sein, für die Bibliotheksnutzer einen entsprechenden Bibliotheksbestand und technische Ausstattung sicherzustellen, was einen nicht geringen finanziellen Aufwand erfordert. Die Sicherstellung der entsprechenden Dienstleistungen stellt die Bibliothekare vor neue Anforderungen, welche bei einem Erfahrungsaustausch mit tschechischen und ausländischen Bibliothekaren verifiziert werden sollten. 


\section{Blanka Konvalinková / Dana Petrýdesová}

Wissenschaftliche Bezirksbibliothek in Liberec

\section{Weshalb haben Sie sich zu der Teilnahme an diesem Projekt entschieden?}

Die Wissenschaftliche Bezirksbibliothek in Liberec war Bestandteil eines vorangegangenen Projektes. Mit der Beteiligung daran waren wir zufrieden. Daher haben wir uns logischerweise dem jetzigen Projekt angeschlossen. Wir erwarteten eine weitere Vertiefung, einen Erfahrungsaustausch und die Möglichkeit, elektronische Medien zu erwerben.

\section{Wie hat Ihre Bibliothek von der Teilnahme am Projekt profitiert?}

Im Projekt haben wir Aktivitäten geplant, welche zu erfüllen waren. Wir schätzen den Erwerb der elektronischen Datenbank Vogue und die Möglichkeit, unseren Vortragsraum mit moderner Technik auszustatten, die die Aufzeichnung der Vorträge und deren Live-Übertragung auf YouTube ermöglicht hat. Wir haben stets Treffen mit allen Projektpartnern bevorzugt. Die Kommunikation mit ihnen war aus professioneller und menschlicher Sicht stets hilfreich.

\section{Welche Projektergebnisse werden Sie in der Bibliothek auch nach Beendigung des Projektes weiterhin nutzen?}

Die oben bereits erwähnte Datenbank und die elektronische Einrichtung im Saal.

\section{Welche Erfahrungen nehmen Sie aus dem Projekt mit? Wollten Sie etwas besser machen oder verbessern?}

Mich freute der große Einsatz beim Leadpartner und an der Hochschule Zittau/ Görlitz. Beide Partner schlossen dem Projekt eine tschechisch sprechende Kollegin an, was den Projektverlauf beträchtlich vereinfachte. Die Aufgabe des Projektmanagements übernahm meine Kollegin, Frau Petrýdesová. Sie arbeitete mit konkreten Aktivitäten und Zahlen. Ich schätzte die Möglichkeit 
der elektronischen Kommunikation, welche uns eine Menge Zeit ersparte. Vielleicht hätte es dem Projekt genützt, wenn es etwas kürzer und intensiver gewesen wäre. Ich merke, dass sich unsere Bibliothek im Charakter von den Universitätsbibliotheken unterscheidet und einige Anregungen und Probleme unsere Bibliothek nicht betreffen und umgekehrt. Auf der anderen Seite konnten wir einen Einblick in das Leben der Universitätsbibliotheken bekommen, weswegen ich das Projekt sehr interessant fand.

\section{Nehmen Sie Potenzial für eine Erweiterung um ein weiteres Projekt wahr?}

Wie bereits gesagt, unterscheidet sich unsere Bibliothek vom Typ her von den anderen am Projekt beteiligten Bibliotheken. Sie kann also den anderen Projektpartnern gegenüber nicht gleichwertig sein. Wenn wir uns einer Fortsetzung des Projektes anschließen sollten, würde ich die Beteiligung unserer Bibliothek eher im Kontext einer weiteren grenzüberschreitenden Kooperation mit Auswirkung nicht nur auf die akademische Öffentlichkeit im grenznahen Gebiet Tschechien - Deutschland sehen. Das Potenzial sehe ich in einer noch engeren Verbindung zwischen den beteiligten Institutionen und den Studierenden. 


\section{Jitka Vencláková}

Universitätsbibliothek der Technischen Universität Liberec

\section{Weshalb haben Sie sich zu der Teilnahme an diesem Projekt entschieden?}

Dies stellte für uns eine gute Gelegenheit dar. Aus einiger Entfernung hatte ich ein Projekt der Projektpartner in der letzten Zeit beobachtet, an dem sich unsere Bibliothek nicht beteiligte. Bei der Vorbereitung des gegenwärtigen Projektes habe ich nicht gezögert, auch unsere Bibliothek einzubinden. Es interessieren mich die Erfahrungen der übrigen Universitätsbibliotheken aus Tschechien und dem Ausland, und die Zusammenarbeit mit diesen ist gewiss ein Gewinn.

\section{Wie hat Ihre Bibliothek von der Teilnahme am Projekt profitiert?}

Durch Erfahrungen. Wir haben eine Menge wertvoller Erfahrungen erworben, die nicht nur die fachlichen Bibliotheksprozesse betreffen (z.B. Fernleihe, institutionelle Repositorien), sondern auch Erfahrungen mit der Veranstaltung von Workshops und ähnlichen Veranstaltungen mit der Teilnahme ausländischer Kollegen. Für mich ist es das erste internationale Projekt, an dem ich teilnehme. Ich habe dabei meine Fähigkeiten als Managerin erprobt. Für unsere Nutzer ist die neue Ausstattung des Lernraums, der ihnen zur freien Verfügung steht, eine große Bereicherung (Kopfhörer, interaktives Panel und Zubehör für Online-Konferenzen).

\section{Welche Projektergebnisse werden Sie in der Bibliothek auch nach Beendigung des Projektes weiterhin nutzen?}

Die Ausstattung. Nach dem Projektabschluss werden die Nutzer auch weiterhin die Möglichkeit haben, die neue Ausstattung des Lernraums zu nutzen. Weitergehen wird das Abonnement der Kollektionen Science \& Technology Library und Business \& Technology der Datenbank des Verlags Taylor \& Francis. Dauerhaft zugänglich werden auch ausgewählte E-Book-Titel aus der 
Datenbank von Taylor \& Francis sein. In unserem Repositorium wird die Publikationstätigkeit der akademischen Mitarbeiter der TUL in übersichtlicher Weise in geeigneter Form zugänglich gemacht. Zur Verfügung steht dann auch eine neue Plattform für Informationskompetenzen, welche neuen, bestehenden und auch potenziellen Nutzern Antworten auf ihre Fragen gibt. Dank den Werbemitteln werden wir auch weiterhin auf die Vorteile und den Sinn, sich dem Projekt im Rahmen des Kooperationsprogrammes zur Förderung der grenzüberschreitenden Zusammenarbeit zwischen dem Freistaat Sachsen und der Tschechischen Republik 2014-2020 und ähnlichen Programmen anzuschließen, hinweisen können.

\section{Welche Erfahrungen nehmen Sie aus dem Projekt mit? Wollten Sie etwas besser machen oder verbessern?}

Die Fähigkeiten. Ich habe mich persönlich darüber vergewissert, dass ich gemeinsam mit meinen Kollegen im Stande bin, ein solches Projekt zu planen, umzusetzen und zu dokumentieren. In dem Moment, in dem alles genau geplant ist und finanzielle und personelle Deckung besteht, ist die Umsetzung eines Projektes viel einfacher.

\section{Nehmen Sie Potenzial für eine Erweiterung um ein weiteres Projekt wahr?}

Ja, ich nehme ein Potenzial wahr. Es wäre beispielsweise interessant, die Medien aller Partner im Rahmen der einzelnen Kataloge zu durchforschen. Gemeinsame Veranstaltungen und Werbung dürften dabei nicht fehlen. 



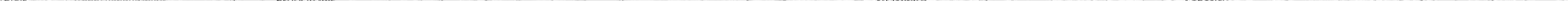





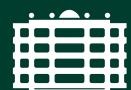

Universitätsbibliothek

TECHNISCHE UNIVERSITÄT

CHEMNITZ

Europäische Union. Europäischer Fonds für regionale Entwicklung. Evropská unie. Evropský fond pro regionální rozvoj.

\section{PROSTOR UČENÍ - KNIHOVNICKÁ INFORMAČNÍ PLATFORMA}

Zkušenosti a výsledky kooperačního projektu knihoven ze Saska a Česka 


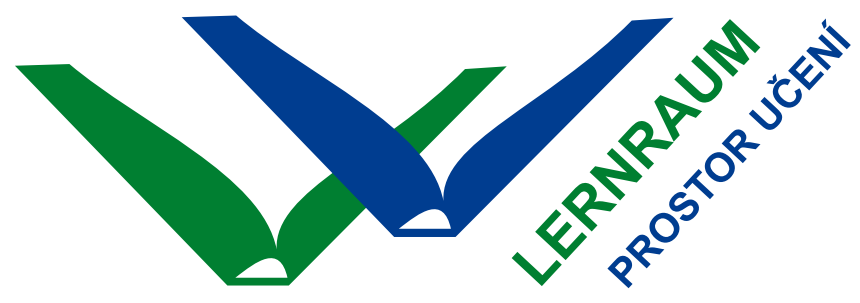

\section{PROSTOR UČENÍ - KNIHOVNICKÁ INFORMAČNÍ PLATFORMA}

Zkušenosti a výsledky kooperačního projektu knihoven ze Saska a Česka

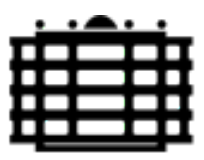

TECHNISCHE UNIVERSITÄT CHEMNITZ

Universitätsverlag Chemnitz 2018 


\section{Tiráž}

\section{Bibliografické informace České národní knihovny}

Česká národní knihovna zařazuje tuto publikaci do České národní bibliografie; přesné bibliografické údaje jsou uvedeny na internetových stránkách: https://www.nkp.cz

\section{Bibliografické informace Německé národní knihovny}

Německá národní knihovna zařazuje tuto publikaci do Německé národní bibliografie; přesné bibliografické údaje jsou uvedeny na internetových stránkách: http://www.dnb.de

Předtiskové úpravy a layout:

Jakub Pokorný, Západočeská univerzita v Plzni / Univerzitní knihovna

Překlad:

Martina Pál, Helena Neumannová

Editorky:

Veronika Jahn, Markéta Siegel, Cornelia Oertel

Titulní obrázek:

Rico Welzel, Technische Universität Chemnitz

Technische Universität Chemnitz / Universitätsbibliothek

\section{Universitätsverlag Chemnitz}

09107 Chemnitz

https://www.tu-chemnitz.de/ub/univerlag

readbox unipress

in der readbox publishing $\mathrm{GmbH}$

Am Hawerkamp 31

48155 Münster

http://unipress.readbox.net

ISBN 978-3-96100-063-0

http://nbn-resolving.de/urn:nbn:de:bsz:ch1-qucosa2-314967

DOI 10.15240/tul/003/9783961000630

https://dspace.tul.cz/handle/15240/26601 
Tato publikace vznikla jako výsledek projektu Prostor učení - Knihovnická informační platforma, který byl podpořen Evropskou unií v rámci Programu na podporu přeshraniční spoluprácemezi Českou republikou a Svobodným státem Sasko 2014-2020.

Na projektu spolupracovalo následujicích pět partnerů:
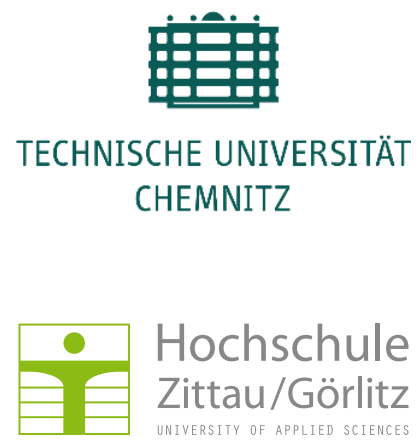

Knibbovna Libraby Liberec $_{\text {Liber }}$

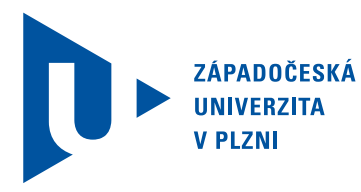

\section{TECHNICKÁ UNIVERZITA V LIBERCI \\ Univerzitní knihovna}

Další informace naleznete na adrese:

https://www.bibliothek.tu-chemnitz.de/lernraum/index.html.cs 


\section{Autoři:}

\section{Angela Malz}

Univerzitní knihovna Technické univerzity Chemnitz

\section{Monika Ullmann}

Univerzitní knihovna Technické univerzity Chemnitz

\section{Falk Maiwald}

Vysokoškolská knihovna Vysoké školy Zittau/Görlitz

\section{Ralf Schwarzbach}

Vysokoškolská knihovna Vysoké školy Zittau/Görlitz

\section{Miloslava Faitová}

Univerzitní knihovna Západočeské univerzity v Plzni

\section{Magda Šrajbová}

Univerzitní knihovna Západočeské univerzity v Plzni

\section{Zdeňka Firstová}

Univerzitní knihovna Západočeské univerzity v Plzni

Miroslava Pourová

Univerzitní knihovna Západočeské univerzity v Plzni

Blanka Konvalinková

Krajská vědecká knihovna v Liberci

Dana Petrýdesová

Krajská vědecká knihovna v Liberci

Jitka Vencláková

Univerzitní knihovna Technické univerzity v Liberci

\section{Václav Ovčačík}

Univerzitní knihovna Technické univerzity v Liberci 


\section{OBSAH}




\section{Falk Maiwald}

1 Prostory učení bez hranic …………………………………………….... 7

Monika Ullmann / Angela Malz

2 Elektronická média a meziknihovní výpůjční služba v přeshraničním konsorciu

Falk Maiwald

3 Mediální technika a infrastruktura v propojeném prostoru učení 29

Zdeňka Firstová / Miroslava Pourová

4 Open Access

Angela Malz / Václav Ovčačík

5 Informační vzdělávání v přeshraničním prostoru učení

59

Dana Petrýdesová / Blanka Konvalinková

6 Poznatky úspěšné spolupráce 69

Angela Malz / Falk Maiwald / Ralf Schwarzbach / Miloslava Faitová / Magda Šrajbová / Blanka Konvalinková / Dana Petrýdesová / Jitka Vencláková 75 otázek pro 5 partnerů 


\section{PROSTORY UČENÍ BEZ HRANIC}

Falk Maiwald 


\subsection{Partneři a cíle projektu}

V rámci Programu na podporu preshraniční spolupráce mezi Českou republikou a Saskem 2014-2020 realizovala Univerzitní knihovna Chemnitz, Vysokoškolská knihovna Zittau/Görlitz, Univerzitní knihovna v Plzni, Krajská vědecká knihovna v Liberci a Univerzitní knihovna v Liberci společný projekt Prostor učení - Knihovnická informační platforma. Tím všech pět projektových partnerů zásadně přispělo k aktuálnímu tématu - knihovny jako prostoru učení. Jinak než u bě̌̌ných diskuzí v knihovnickém sektoru nebyla pozornost zaměřena na vlastní prostor učení. Hlavním cílem projektu bylo především vzdělávání ve společném, udržitelném přeshraničním prostoru učení. Všechny snahy projektu byly zaměřeny na tento cíl. Pro postupné a úspěšné zvládnutí tohoto komplexního záměru, bylo od všech projektových partnerů zapotřebí

- Stanovení jasně vymezených milníků (viz kapitola 1.4),

- sladěné, strukturované jednání,

- vzájemné porozumění a

- akceptování kroků a opatření v rámci jednotlivých milníků projektu.

Výchozím bodem byla základní koncepce k významu a obecnému obsahu projektu na téma prostor učení (viz kapitola 1.3) i jednotlivým milníkủm (viz kapitola 1.4). Stejně jako u většiny ostatních projektů závisí úspěch i tohoto projektu na jejich aktérech a ochotě spolupracovat. Dále je důležité upřesnit, jaký prínos resp. jaké efekty by měly být během projektu dosaženy (viz kapitola 1.2).

\subsection{Spolupráce jako základ pro přeshraniční knihovnický prostor učení}

Spolupráce mezi knihovnami na regionální až národní úrovni jsou jak v České republice tak v Sasku resp. v Německu již dlouho dobu začleněny do různých tématických oblastí. Dưvody pro spolupráci vědeckých knihoven jsou např. vzájemná tvorba a výměna znalostí a zkušeností nebo využití efektů díky spojenectvím za účelem využívání a řízení zdrojů (např. pořizování elektronických médií, meziknihovní výpůjční služba, semestrální aparáty). Stejné důvody platí i v př́padě mezinárodních kooperací mezi knihovnami, přestože zde mohou významnou roli hrát obsahové, kulturní, politické či právní rozdíly. 
V přihraničí se nabízí přeshraniční spolupráce na základě geografické polohy potencionálních kooperačních partnerů. Předpokladem je, aby uživatelé našli ve svém spádovém regionu na obou stranách hranice relevantní vědecké knihovny, jako je tomu např. v Zittau resp. v Liberci. Proto nikoho nepřekvapí, že již řadu let existují úzké kontakty a aktivní spolupráce mezi Vysokoškolskou knihovnou Zittau/Görlitz na jedné a Krajskou vědeckou knihovnou a Univerzitní knihovnou v Liberci na druhé straně. ${ }^{1}$ Přeshraniční spolupráce mezi knihovnami může být navíc založena na kooperaci př́slušných vysokých škol resp. univerzit, jako např. v podobě semestrálních aparátů Univerzitní knihovny Chemnitz a Univerzitní knihovny Západočeské univerzity v Plzni.²

Tyto dlouhodobě zaměrené bi- a trilaterální spolky budou i nadále existovat at v této nebo jiné, podobné formě. Odhodlání ke společným přeshraničním aktivitám, vzájemný respekt a prátelská důvěra totiž posilují tato spojení a zůstanou zachovány. Vedle toho ale dochází k situacím, kdy je dočasná, krátkodobější spolupráce s dalšími partnery, kteři řeší podobné problémy nebo hledají odpovědi na podobné otázky, ve formě projektů smysluplná.

Již v roce 2009 odstartovala Univerzitní knihovna Chemnitz projekt s Univerzitou Jana Evangelisty Purkyně (UJEP) v Ústí nad Labem a Univerzitní knihovnou Západočeské univerzity v Plzni. ${ }^{3} \mathrm{U}$ této prvotní spolupráce na projektu mezi sousedními knihovnami byla hlavním cílem výstavba Česko-saské odborné knihovny a přeshraniční meziknihovní výpůjucní služba. V letech 2013-2014 následoval projekt Prostor učení - Knihovnická informační platforma. Projektovými partnery byla Univerzitní knihovna Technické univerzity Chemnitz, Vysokoškolská knihovna Zittau/Görlitz, Univerzitní knihovna Západočeské univerzity v Plzni a Krajská vědecká knihovna v Liberci. Cílem projektu bylo vytvoření dlouhodobé efektivní sítě knihoven mezi Českou republikou a Saskem.

Díky zde prezentovanému projektu Prostor učení - Knihovnická informační platforma se spolupráce dále prohloubila - tentokrát za účasti Univerzitní knihovny Technické univerzity v Liberci a s velmi ambiciózními cíli a úkoly v oblasti „prostoru učení".

\footnotetext{
Srov. Maiwald (2016).

2 Srov. Slabý (2014).

3 Srov. Malz (2010).
} 


\section{Prínos spolupráce a projektu}

Hned na začátku projektu, v rámci zahajovací akce v lednu 2017 diskutovali projektoví partneři očekávaný př́inos resp. šance projektu, přičemž rozlišovali interní a externí efekty.

Mezi nejdůležitější interní faktory mající vliv na kooperující knihovny patřili

- bezprostřední teritoriální blízkost,

- podobná témata a problémy a jejich odpovídající řešení,

- učení se od sebe - učení se spolu - učení se o sobě,

- posílení „značky knihovna“ (výkonnost, up to date) v rámci vysoké školy,

- posílení vztahů mezi vysokoškolskými knihovnami - průkopníky v oblasti prostoru učení,

- odstraňování „překážek“ a nízkoprahový přistup k dalším knihovnám.

Jako externí efekty, tzn. efekty působící v celém příhraničí i nad jeho rámec, očekávali projektoví partneři

- rozšrirení spektra nabídky v souvislosti se spoluprací („vytváření mostů“) díky moderním infrastrukturám a společně využívaným médiím (elektronické knihy a meziknihovní výpůjční služba),

- zlepšení informační kompetence uživatelů,

- zvýšení spokojenosti uživatelůa

- tím vliv na pozitivní postavení vlastní vysoké školy u potencionálních studentů a multiplikátorů (rodiče, učitelé),

- posílení vědeckého prostředí v regionu,

- posílení myšlenky Open Access,

- posílení vnímání a image „značky knihovna“,

- referenční charakter, tzn. sít knihoven jako průkopník přeshraniční spolupráce a vzor pro prostory učení dalších knihoven.

\subsection{Prostory učení vědeckých knihoven a prostor učení projektu}

„Prostory učení propojují všechny zdroje, služby, opatření a infrastrukturu pro vytváření studijní a pracovní situace studentü“ ${ }^{4}$, kteří tento prostor „V časově omezeném době za účelem učení vyhledávají" ${ }^{\prime 5}$. Vědecké knihovny jako

4 Srov. DINI (2013), str. 7.

5 Srov. May/Kannenberg (2014), str. 9. 
prostory učení se v minulých letech dočkaly významného uznání. Na jedné straně je tento vývoj podmíněn měnícími se formami učení jako jsou např. projektové a skupinové práce, blended learning a koncentrovaná dostupnost tištěných a elektronických médií na jednom místě, na straně druhé se v dnešní digitální době zásadně změnily potřeby a nároky studentů.

Adekvátně k tomuto vývoji se chovají i vědecké knihovny, které směřují utváření svých prostorů učení bliže k požadavkům uživatelů. S cílem podpořit "nové" učení vznikají rozmanité koncepty na mírứ. Digitální technologie a média přitom hrají velmi důležitou roli. Tradiční knihovna jako místo pro knihy se změnila v místo pro nejrůznější interní a externí skupiny uživatelů, kteří v knihovně pracují, komunikují, vyvíjejí nové nápady nebo se „jen“ setkávají.

\section{Typologie skupin uživatelů vědeckých knihoven}

K interním uživatelům (viz obrázek 1) patří v první řadě studenti a studentky. V prostoru učení knihovny pracují ale také výzkumní zaměstnanci a vyučující. Hlavním cílem prostoru učení je cílená podpora a další rozvoj učebních procesů díky utváření učebního prostředî?, a proto se tento proces týká i „učící se organizace" ${ }^{\prime \prime}$ - knihovny samotné. Z tohoto důvodu je nezbytné zohlednit
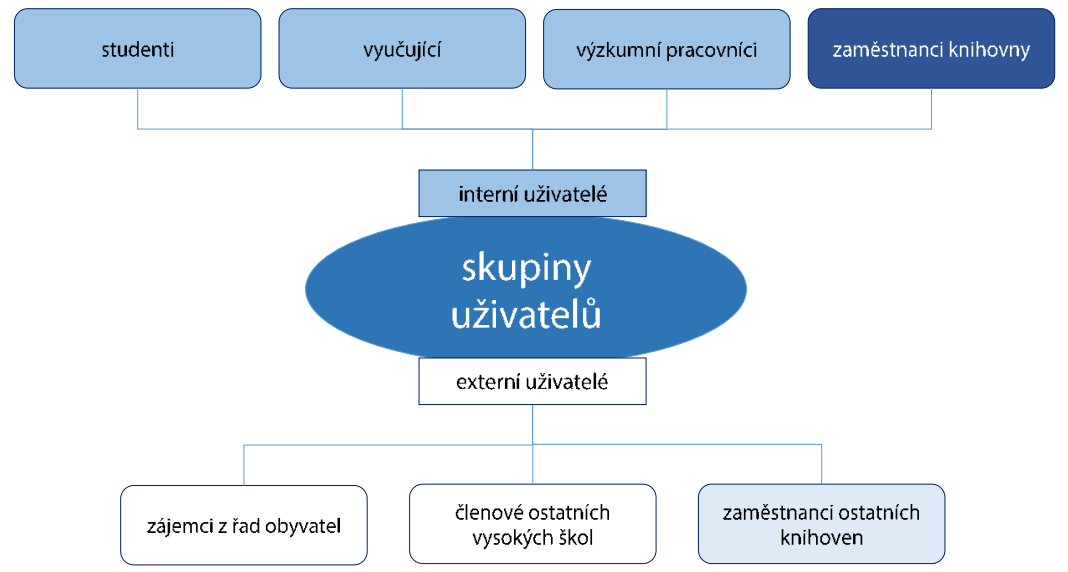

Obrázek 1. Typologie skupin uživatelů v prostoru učení vědecké knihovny. Zdroj: vlastní zpracování.

${ }^{6}$ Srov. zast. Kohl-Frey (2014), Rautenberg et al. (2014).

7 Srov. May/Kannenberg, 2014, str. 10.

${ }^{8}$ Podle Senge (2011). 
v širším smyslu také procesy učení zaměstnanců a zaměstnankyň knihoven, využívajících prostor učení k dalšímu vzdělávání a výměně odborných znalostí a zkušeností. Toto pojetí je díky svému praktickému významu při kooperaci (vgl. kapitola 1.2) pro tento projekt velmi důležité.

Do externích skupin uživatelů Ize v úzkém smyslu zahrnout zainteresované osoby z široké veřejnosti nebo příslušníky jiných vysokých škol, tedy studenty, vyučující a výzkumné pracovníky. V širším smyslu sem patří rovněž zaměstnanci knihovny.

\section{Základní typy prostorů učení}

Zohledňujeme-li prostory učení z různých perspektiv, Ize je podle Wittwera/ Diettricha (2015) rozdělit na materiálně-fyzické, sociální, virtuální a kooperační prostory. ${ }^{9}$ Ze sociálního hlediska jsou prostory učení místy setkávání a tím sociálních interakcí. Na druhé straně vyvolávají ale také očekávání, která uživatelé spojují s děním na tomto místě ${ }^{10}$

V materiálně-fyzickém kontextu představují prostory učení reálné, do sebe uzavrené fyzické jednotky - zpravidla v rámci jedné nebo několika budov - vybavené infrastrukturou odpovídající účelu využití. Patří sem např. nábytek, technika a další prvky pro vybavení prostoru. ${ }^{11} \mathrm{~V}$ následujícím textu budou materiálně-fyzické prostory učení se zohledněním sociálních komponent označovány jako lokální prostory učení.

Virtuální prostory učení mají být v této souvislosti chápány jako nefyzické prostory učení, u nichž neplatí jednota prostoru a místa a komunikace probíhá přes média. Jejich výchozím předpokladem jsou sice materiálně-fyzické resp. Iokální učební prostory, ty však nejsou vázány na žádné fyzické hranice prostoru. S pomocí telekomunikačních a internetových technologií mohou mít "globální" charakter, tzn. mohou překračovat geografické a (trvalé) politické hranice. V tomto kontextu budou v této práci označovány jako virtuální prostory učení propojující několik pracovišt'. Stejně jako lokální prostory učení se skládají ze sociálních resp. interkulturelních dimenzí s vlastními hodnotovými vzorci a pravidly chování. ${ }^{12}$

\footnotetext{
9 Srov. Wittwer/Diettrich (2015), str. 15nn.

10 Srov. May/Kannenberg (2014), str. 10.

11 Srov. May/Kannenberg (2014), str. 10.

12 Srov. Wittwer/Diettrich (2015), str. 19, str. 21.
} 
Díky různým aktérů, jejich interakcím a společné práci přispívají kooperační místnosti - obvykle v rámci sítí nebo (modelových) projektů - ke společnému řešení problémů. Spolupráce (viz kapitola 1.2) vznikají, rozrůstají se a dále se rozvijí v rámci určitého kooperačního procesu, což vede $\mathrm{k}$ odlišnému způsobu jednání, práce a učení se než v jiných sociálních místnostech. ${ }^{13}$

\section{Vymezení a inovace prostoru učení v projektu}

$\checkmark$ projektu se prakticky propojily všechny perspektivy prostoru učení. Se zaměřením na hlavní cíl bylo zásadním úkolem popsat a vytvoriit preshraniční prostor učení pro spolupráci resp. propojení mezi českými a saskými projektovými partnery. Na rozdíl od klasického přístupu (viz obrázek 1) bylo třeba zapojit potencionálně spolupůsobící skupiny uživatel všech kooperačních partnerů (obrázek 2), identifikovat společné učební zájmy subprostorů učení a vyvinout obsahová témata a technická řešení pro lokální a meziorganizační prostor učení. Jako výsledek měla vzniknout Knihovnická informační platforma.

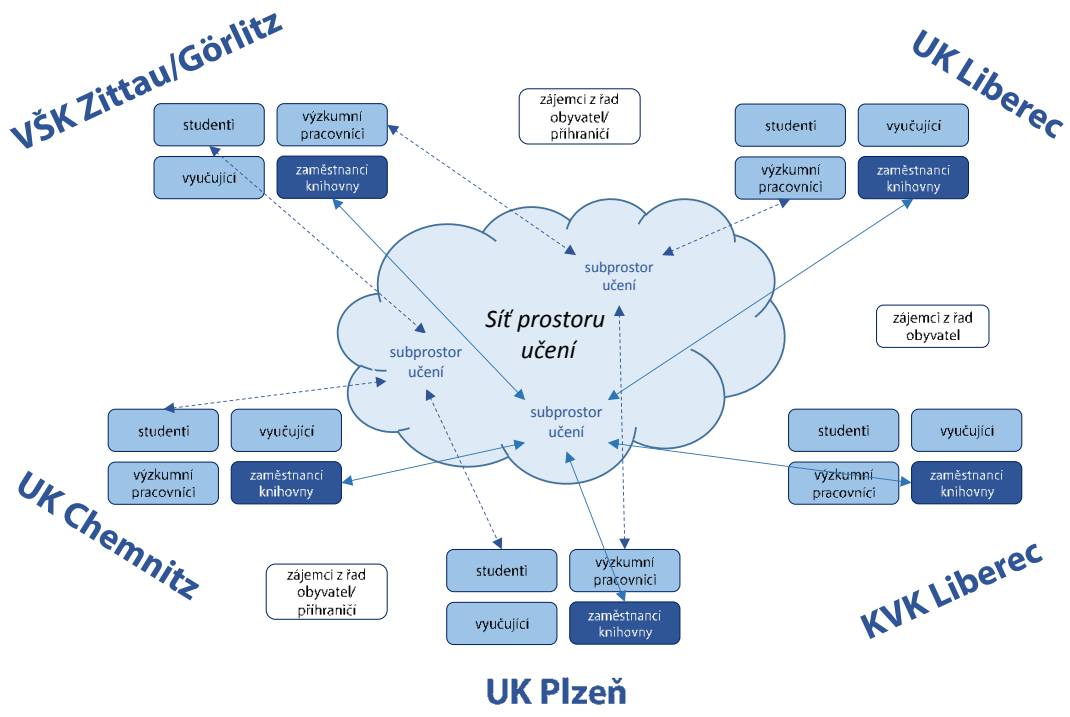

Obrázek 2. Schéma sítě prostoru učení a jeho subprostory. Zdroj: vlastní zpracování.

Pod pojmem meziorganizační sít prostoru učení se rozumí neformální propojení studentů, žáků vyučujících, výzkumných pracovníkủ a/nebo zaměstnanců

${ }^{13}$ Srov. Wittwer/Diettrich (2015), str. 21. 
knihovny, kteří na základě společných zájmů a problémů spolupracují, vyměňují si znalosti a zkušenosti a přitom se od sebe učí. ${ }^{14}$ Touto definicí se ve srovnání s předchozími projekty významně rozšrîiil horizont projektu (viz kapitola 1.2). Velkou roli totiž nehraje „pouze“ samotná knihovnická práce, ale také vztahy a spolupráce mezi ostatními aktéry vysokých škol a univerzit.

Materiálně-fyzický meziorganizační prostor učení lze realizovat pouze ve formě setkávání na stejném místě a ve stejný čas, např. pro porady, odborné konference, workshopy apod. V projektu podobné akce několikrát proběhly ve formě různých prezenčních aktivit za účasti různých skupin uživatelů, vždy v souladu s daným tématem. Zatímco se praktických stáží a workshopů v jednotlivých milnících účastnili zaměstnanci projektu a knihovny, byly akce k informačnímu vzdělávání určené především pro studenty, vyučující a výzkumné pracovníky. Pořádající projektový partner vždy pro tato setkání poskytl svůj lokální prostor učení.

Inovativním elementem projektu ale bylo vytvoření virtuálního meziorganizačního prostoru učení. Podle aktuálních poznatků projektových partnerů podobný prostor učení mezi knihovnami v Sasku a České republice, ani nikde jinde doposud neexistoval. Takový virtuální prostor může v principu fungovat jen tehdy, když spolu zúčastněné skupiny uživatelů komunikují a spolupracují přes internet a ruší tak prímé propojení v rámci prostoru resp. času. V projektu Prostor učení byly webové konference využívány jako typická forma komunikace mezi různými místy ve stejný čas, především pro koordinaci projektu mezi knihovnami, ale také pro spolupráci mezi ostatními skupinami uživatelů. Pro časově oddělenou přeshraničí komunikaci resp. spolupráci byly využívány e-learningové systémy a internetové platformy (viz kapitola 5), emaily a centrální datová média pro zálohování dat. Technické předpoklady pro lokální prostory učení jednotlivých partnerů byly využity resp. pořízeny v rámci milníku 3 (viz kapitola 3).

\subsection{Odvození projektových obsahů v přeshraničním prostoru učení mezi knihovnami}

Vysoká očekávání od projektu (viz kapitola 1.2) vyplývají z milníků projektu, které byly závazně stanoveny již v projektové žádosti.

14 Podle Kupfer (2015), str. 204. 
Jedná se 0:

1. Konsorciální pořízení elektronických informačních zdrojů; meziknihovní výpůjční služba a semestrální aparáty

2. Technické vybavení prostoru učení

3. Open Access

4. Odborné zprostředkování informační kompetence

5. Akce a opatření pro práci s veřejností

Nejvýše postavený cíl v milníku 1 byla iniciační realizace česko-saského konsorcia pro pořizování elektronických knih, které měly být v prostoru učení k dispozici všem uživatelům na obou stranách hranice. Hlavní úlohu při navazování kontaktů a jednání s nakladatelstvími převzal lead partner, Univerzitní knihovna Chemnitz. Dalším důležitým cílem bylo rozšiření přeshraniční meziknihovní výpůjční služby, spojené s analýzou potřeb a možností řešení semestrálních aparátů (viz kapitola 2).

V milníku 2 si Vysokoškolská knihovna Zittau/Görlitz vzala za úkol vytvořit koncept pro inovativní jádro virtuálního přeshraničního prostoru učení, a to na základě analýzy možných scénářủ využití mezi skupinami uživatelů. Výsledkem bylo odvození plánu, pořízení a implementace odpovídající počítačové a mediální techniky, interaktivních tabulí a konferenční techniky. Následující krok představovalo úspěšné zavedení virtuálního prostoru učení knihoven (viz kapitola 3).

Další rozvoj myšlenky Open Access, tzn. neomezeného, bezplatného př́ístupu k vědeckým informacím, a intenzivnější propojení repozitářů mezi partnery v milníku 3 zodpovědně převzala Univerzitní knihovna Západočeské univerzity v Plzni. Důležitým cílem v této souvislosti byla vzájemná výměna informací ohledně pokroku v aktivitách týkajících se Open Access zúčastněných vysokoškolských knihoven a další senzibilizace potencionálních autorů a autorek (viz kapitola 4).

Zvyšování informační kompetence uživatelů, tedy schopnosti najít, zpracovat a vyhodnotit informace, je stálým tématem všech knihoven. V milníku 4, za který převzala zodpovědnost Krajská vědecká knihovna a Univerzitní knihovna v Liberci, bylo klíčovou snahou lépe zaintegrovat informační vzdělávání do výuky, výzkumu a studia. K tomuto cíli má významně přispět speciálně vyvinutý, dvojjazyčný výukový modul k informační kompetenci v lokálním a přeshraničním prostoru učení - tzn. v souvislosti s činnostmi všech projektových partnerů. Předmětem diskuzí v rámci projektu byly mimoto i další koncepty (viz kapitola 5). 
Centrálním bodem milníku 5 byla tato, již hotová publikace, která dokumentuje práci na projektu, výsledky projektu i vytvořenou přidanou hodnotu. V ideálním prípadě navíc slouží - alespoň zčásti - jako orientace pro podobné záměry dalších knihoven.

\section{Použitá literatura}

DINI, Deutsche Initiative für Netzwerkinformation e.V.: Die Hochschule zum Lernraum entwickeln, Kassel 2013.

May, A.; Kannenberg, S.: Entgrenzung und Zusammenarbeit - die Notwendigkeit von Kooperationen im Lernraum; in: ABI Technik, sešit 34 (1) 2014, str. 9-19.

Kohl-Frey, 0.: Die Universitätsbibliothek als neuer Lernraum: Konzept der Universität Konstanz; in: Eigenbrodt, 0.; Stang, R. (vyd.): Formierungen von Wissensräumen. Optionen des Zugangs zu Information und Bildung; Berlin/ Boston 2014, str. 107-123.

Kupfer, F.: Lernen und Arbeiten in Lernort übergreifenden Netzwerken, in: Wittwer, W.; Diettrich, A.; Walber, M. (Hrsg.): Lernräume, Wiesbaden 2015, str. 204-217.

Maiwald, F.: Internationale Kooperationen im Dreiländereck; in: BIS - Das Magazin der Bibliotheken in Sachsen, Dresden: Sächsische Landesbibliothek - Staats- und Universitätsbibliothek Dresden, č. 3/2016, str. 168-169.

Malz, A.: Grenzüberschreitende Kooperation: Die Universitätsbibliotheken in Chemnitz, Plzen und Ústí nad Labem arbeiten zusammen; in: BIS - Das Magazin der Bibliotheken in Sachsen, Dresden: Sächsische Landesbibliothek - Staats- und Universitätsbibliothek Dresden, č. 4/2010, str. 254-255.

Rautenberg, K.; Klein, A.; Usinger, J.; Dombrowe, A.: Vom Lesesaal zum Learning Center - Ein neues Raum- und Benutzungskonzept an der UB Mannheim; in: b.i.t. online, č. 4/2014, str. 321-328.

Senge, P: Die fünfte Disziplin. Kunst und Praxis der lernenden Organisation. Klett-Cotta, 11. vydání 2011.

Slabý, Adam: Damit einschlägige Werke verfügbar werden, 2014, dostupné z: https://www.tu-chemnitz.de/tu/pressestelle/aktuell/5960, poslední prístup 22.08.2018

Wittwer, W.; Diettrich, A.: Zur Komplexität des Raumbegriffs; In: Wittwer, W.; Diettrich, A.; Walber, M. (Hrsg.): Lernräume. Gestaltung von Lernumgebungen für Weiterbildung; Wiesbaden 2015, str. 1127. 


\section{ELEKTRONICKÁ MÉDIA A MEZIKNIHOVNÍ VÝPŮJČNÍ SLUŽBA V PR̆ESHRANIČNÍM KONSORCIU}

Monika Ullmann

Angela Malz 


\section{1 Česko-saská odborná knihovna jako předchůdce přeshraničního konsorcia}

Česko-saské projekty na Technické univerzitě Chemnitz začaly již dávno před projektem Prostor učení - Knihovnická informační platforma. Byly úzce spojeny s bohužel př́liš brzy zesnulým panem prof. Dr. Peterem Jurczekem, který tuto spolupráci aktivně inicioval. Od začátku byly vyčleněny prostředky k vytvoření Česko-saské odborné knihovny.

Roku 2003 stálo na začátku Česko-saské vysokoškolské centrum (ČSVC). Cílem projektu bylo vybudování dlouhodobé vědecké spolupráce mezi vysokými školami v česko-saském príhraničí. Projekt byl spolufinancován z prostředků Iniciativy společenství Evropské unie Interreg III A.

V rámci tohoto projektu získala Univerzitní knihovna Chemnitz prostředky na vybudování Česko-saské odborné knihovny (ČSOK). Knihovní fond sloužil k podpoře přeshraniční vědecké spolupráce mezi Českou republikou a Saskem. Univerzitní knihovna Chemnitz pořídila knihy v německém a českém jazyce s tématikou česko-německé spolupráce. Ty pak rozšririla o odborné knihy z oblasti ekonomie, dějin Saska a Česka nebo rož̌îrení Evropské Unie na východ. Do fondu přibyla také beletristická díla, jazykové a turistické průvodce, mapy, DVD nebo audioknihy. ${ }^{15} \mathrm{~V}$ současné době tato odborná knihovna zahrnuje zhruba 11123 médií.

V roce 2006 projekt ČSVC skončil. V následném projektu Česko-saské vysokoškolské kolegium, který byl rovněž spolufinancován z prostředků Evropské unie, získala odborná knihovna opět k dispozici další prostředky.

V létě 2009 odstartoval projekt Česko-saská vysokoškolská iniciativa. Spolufinancován byl z programu Cíl 3 z Evropského fondu pro regionální rozvoj, z prostředků Svobodného státu Sasko, České republiky a zúčastněných univerzit.

Zde poprvé hrály knihovny zúčastněných univerzit v projektové žádosti významnější roli. Vedle zařízení Česko-saské odborné knihovny byl kladen dưraz na přeshraniční výpůjční službu a posílení spolupráce mezi knihovnami.

Partnery Univerzitní knihovny Chemnitz byla Knihovna Univerzity Jana Evangelisty Purkyně (UJEP) v Ústí nad Labem a Univerzitní knihovna Západočeské univerzity v Plzni.

15 https://www.tu-chemnitz.de/ub/STHZ/sthz_medien.html 
Na konci projektu bylo již zřejmé, že knihovny chtějí spolupracovat i nadále a zapojit do česko-saského príhraničí i další knihovny. Díky tomu vznikl projekt Česko-saská sit knihoven - informace bez hranic, který byl realizován od 01.12.2013 do 30.11.2014. Projektovými partnery byla Univerzitní knihovna Technická univerzita Chemnitz, Vysokoškolská knihovna Zittau/Görlitz, Univerzitní knihovna Západočeské univerzity v Plzni a Krajská vědecká knihovna v Liberci. Cílem projektu bylo vytvoření udř̌itelné a efektivní sítě knihoven mezi Českou republikou a Saskem.

Pozitivní zkušenosti a osobní kontakty navázané $v$ tomto projektu vedly v roce 2015 k rozhodnutí dále spolupracovat v novém projektu Prostor učení - Knihovnická informační platforma v rámci Programu na podporu preshraniční spolupráce mezi Českou republikou a Svobodným státem Sasko 2014-2020. Projektovými partnery byla Univerzitní knihovna Technické univerzity Chemnitz, Vysokoškolská knihovna Zittau/Görlitz, Univerzitní knihovna Západočeské univerzity v Plzni, Univerzitní knihovna Technické univerzity v Liberci a Krajská vědecká knihovna v Liberci.

\subsection{Milník 1 projektu Prostor učení - Knihovnická informační platforma}

Cílem nového projektu bylo dosáhnout toho, co v minulých projektech bylo sice častým tématem rozhovorů, ale kvůli krátké době trvání projektu nebylo možné realizovat: vytvoření prvního česko-saského konsorcia pro pořizování elektronických informačních zdrojü. V rámci tohoto konsorcia se sdružily české a saské knihovny a vytvoriily společenství s cílem získat lepší nákupní podmínky, než jaké by mohly vyjednat jako samostatný pořizovatel. Zvláštností projektu Prostor učení pritom bylo přeshraniční využívání pořízených zdrojů. Tato média mohli za stejných podmínek využívat uživatelé všech projektových knihoven na obou stranách hranice.

Tištěné knihy v rámci Česko-saské odborné knihovny v Univerzitní knihovně Chemnitz a všechny dalši tištěné zdroje si mohou uživatelé českých knihoven vypưjčit přes přeshraniční meziknihovní výpưjční službu. Mimoto jsou k dispozici pro semestrální aparáty.

Projekt Prostor učení - Knihovnická informační platforma stavěl na jedné straně na výsledcích předchozích projektů a dále je prohloubil. Česko-saská odborná knihovna se svými tištěnými médii byla i nadále významnou složku spolupráce. 
Na druhé straně zohlednily knihovny požadavky na digitální knihovny a pořídily nové elektronické informační zdroje.

V projektové žádosti v tomto kontextu stojí: „Prostor učení staví na existujících zdrojích znalostí. Knihovny vytvoří konsorcium a pořídí nové elektronické informační zdroje - e-knihy, která mohou přeshraničně využívat všichni uživatelé zúčastněných knihoven. Díky společnému nákupu ve větším množství může konsorcium získat výhodnějších cenové podmínky. Také tyto informační zdroje budou v rámci konsorcia součástí meziknihovní výpůjční služby a elektronického zasílání dokumentů. Tištěná média budou projektovým partnerům zasílána pomocí přeshraniční meziknihovní výpůjční služby. Podle přání a potřeby výuky budou knihy poskytnuty k dispozici také pro semestrální aparáty."

Aktivity k dosažení těchto cílů:

- Workshop ve Vysokoškolské knihovně v Zittau 29.03.2017

- Praktická stáž v Univerzitní knihovně Chemnitz 04. až 07.12.2017

- Workshop v Univerzitní knihovně v PIzni 14.02.2018

- Workshop v Krajské vědecké knihovně v Liberci 23.05.2018

Při přípravě Česko-saského konsorcia pro pořizování elektronických médií byly přezkoumány možnosti konsorciálního nákupu v Česku i v Německu.

Elektronická média Ize v Česku z velké části pořídit pouze v rámci projektů tzn. z dotačních prostředků. Podobné modely pro pořizování jako v Německu nejsou realizovatelné. Z tohoto důvodu bylo rozhodnuto, že vedoucím konsorcia bude německá knihovna a volba padla přímo na lead partnera, Univerzitní knihovnu Chemnitz.

\subsection{Konsorcia v Německu}

V Německu existuje několik konsorciálních modelů.

V letech 2004 až 2010 byl nadací Die Deutsche Forschungsgemeinschaft (DFG) (Německá výzkumná společnost) žrizen program národních licencí. Z prostředků DFG bylo podporováno pořizování uzavřených, elektronických informačních zdrojů s trvalými právy na archivaci a hosting: databáze plných textů, archivy časopisů, sbírky elektronických knih (e-knih). V tomto programu bylo pořízeno celkem přes 140 národních licencí za více než 100 milionů eur. Dotační prostředky poskytnuté DFG pokryly všechny náklady. 
K podporovaným projektům patřily např.:

- Early English Books Online

- NetLibrary

- The Making of the Modern World: economics, politics and industry

- Cambridge Journals Archives 1864-1996

- Springer Online Journals Archives 1860-2000

- Taylor \& Francis Online Archives 1799-2000

Zúčastnit se mohli všichni uživatelé vědeckých knihoven i zainteresovaní soukromí uživatelé z Německa. Účastníci se museli zavázat, že budou akceptovat vyjednané uživatelské podmínky. Hlavní úlohu při vyjednávání nehrála DFG, ale knihovny z okruhu bývalé speciální sběrné oblasti. Knihovny prevzzaly jednání s jednotlivými nakladatelstvími, podání žádosti, získání a uzavření licenčních smluv. Mimoto byly zodpovědné za dlouhodobé zprístupňování produktů v rámci celého Německa. Hlavními vyjednávajícími byly:

- Bavorská městská knihovna

- Německá lékařská centrální knihovna

- Německá ekonomická centrální knihovna

- Dolnosaská státní a univerzitní knihovna Göttingen

- Státní knihovna v Berlíně - pruské kulturní dědictví

- Technická informační knihovna

- Univerzitní knihovna Johann Christian Senckenberg

Pro licencování pokračujících obsahů byl v letech 2008-2010 otestován pilotní projekt pro pokračujíć časopisy.

Díky knihovnám, které vedly jednání již u národních licencí, došlo ke schválení dvanácti dalších licencí. Ty byly realizovány podle dvou modelů:

Model 1 byl zaměřen na národní licence. Byly uzavřeny plošné a plně financované licence, pričemž se výslovně jednalo o produkty špičkového zabezpečování. PIné financování proběhlo ve finančně omezené oblasti vysoce specializovaných zdrojů. Okruh účastníků byl stejný jako u klasických národních licencí.

K podporovaným produktům patřily:

- China Academic Journal

- Editoria Italiana Online Periodici

- Kluwer Law Journals 
Ve 2. modelu - tzv. „opt-in-modelu“ byla nutná spoluúčast zúčastněných. Ta nepřipouštěla plošné zabezpečování ani individuální přístup jednotlivých uživatelů. Jednalo se tak o nadregionální konsorcium s dotační podporou DFG. Podílem zúčastněných institucí bylo zachování předplatného, aktivního v době uzavření smlouvy. Př́platek za přístup k hromadné nabídce převzala nadace DFG. Instituce bez vlastního předplatného získaly kompletní dotaci.

V prípadě licencovaných balíčků se jednalo o produkty malých nebo středně velkých nakladatelství:

- American Institut of Physics

- Annual Reviews

- British Medical Journal Publishing Group

- Institut of Physics Publishing

- Karger Publishers

- Oxford University Press

- Royal Society of Chemistry

- Sage Publications

- De Gruyter

Jako doplněk k uživatelským podmínkám pro národní licence byl zaveden koncept moving wall. U ročníků licencovaných platícími účastníky byly tyto licence uděleny s jedno- či dlouletým zpožděním ve formě plošné národní licence.

S koncem podpory národních licencí a ukončením pilotního projektu pro pokračující časopisy přešla dotace DFG od roku 2011 v prioritní iniciativu „Digitální informace" vědecké organizace Aliance německých vědeckých institucí. Cílem programu je udržitelná podpora výzkumu a zajištění konkurenceschopnosti Německa jako vědeckého centra.

Do okruhu činností iniciativy Aliance spadá vedle udělování národních licencí také národní hostingové strategie, informace z výzkumu, Open Access, právní rámcové podmínky a virtuální výzkumné prostředí.

S tímto cílem byla založena pracovní skupina Aliance AS Licence (Allianz-AG Lizenzen). Tato skupina se skládá ze zástupců vědeckých organizací podílejících se na iniciativě Aliance a z nadací DFG jmenovaných zástupců knihoven, které se podílejí na vyjednávání v souvislosti s národními licencemi.

Osvědčený model opt-in byl poté otevřen nejen pro časopisy, ale také pro nejrůznější dynamické zdroje. 
Dotační kvóta DFG činila max. 25 \%.

Přechod k modelům nezávislým na výši fondu se ukázal jako velmi složitý. Bylo potřeba definovat parametry, které nebyly orientované na stav odběru, jako např. uživatelé (FTE), využití, rozpočet.

Zásada pořizování médií s dlouhodobým využitím platila i v případě licencí. Sem spadal také moving wall - konkrétní vyjednaná časová hranice pro zveřejnění média.

Aliance AS Licence zhodnotila návrhy produktů předložené knihovnami podle následujićích kritérií:

- Shoda se zásadami pořizování platnými pro národní licence dotované DFG

- Počet návrhů/ naléhavost poť̌eby

- Dostupnost v Německu (střední pokrytí, žádná kapacita, žádné nasycení trhu)

- Aspekty náklady - užitek

- Finanční proveditelnost

- Potenciál úspěchu jednání

U elektronických časopisů patřila k předepsaným komponentům licencí Aliance také složka Open Access. Cílem bylo prosazení zelené cesty Open Access, tedy možnosti préeírat licencované články do institucionálních nebo odborných repozitáruu, a to nejlépe ve formátu uveřejněném nakladatelstvím. Pro instituce a autory je $\mathrm{k}$ dispozici přehled produktů k využívání vyjednaných práv Open Access.

Príklady licencí Aliance:

- Brill Law Books online

- Cambridge Books Online

- MathSciNet - American Mathematical Society

- Nomos eBooks

Gasco (German, Austrian and Swiss Consortia Organisation) - pracovní společenství německých, rakouských a švýcarských konsorcií - propojuje regionální aktivity konsorcií v německy mluvících zemích a vytvárí platformu pro strategickou spolupráci knihoven při společném pořizování elektronických časopisů, databází a elektronických knih.

V Německu existují regionální a institucionální konsorcia. Institucionální konsorcia mají původ v oblasti velkých výzkumných aliancí (společnost 
Fraunhofer, institut Leibniz atd.) a resortního výzkumu v oblasti spolkových ministerstev.

Regionální konsorcia jsou tvořena univerzitními, vysokoškolskými a zemskými knihovnami dané spolkové země. Se souhlasem poskytovatele mohou být zapojeny další vysokoškolské instituce jiných spolkových zemí. Spolkové země s vysokou koncentrací často nadregionálně aktivních univerzit a vysokých škol mívají velká a výkonná konsorcia s vlastním zastoupením. Pobočky s vlastním personálem odebírají část úkolů hlavnímu vyjednávajícímu a jsou pro zvládání velkých konsorcií velmi důležité.

Saské vědecké knihovny tvoři Saské konsorcium. Jednání probíhají vždy v souvislosti s daným produktem za účasti přidružených univerzitních a vysokoškolských knihoven. Konsortium nemá žádnou vlastní pobočku.

\section{4 Česko-saské konsorcium}

Pro konsorcium vytvořené v rámci projektu Prostor učení - Knihovnická informační platforma musely být splněny následující podmínky:

- Najít nakladatelství, která byla ochotná poskytnout nabídku pro přeshraniční konsorcium.

- Média musela odpovídat odborným profilům všech zúčastněných projektových partnerů.

- Musela existovat možnost využívat aktuální tituly.

- Nakladatelství musela mít mezinárodní zaměření.

- Nesmělo dojít ke střetu zájmů se Saským konsorciem.

Na výběr byla tři mezinárodně aktivní nakladatelství Cambridge University Press, Sage Publications a skupina nakladatelství Taylor \& Francis. První jmenované pokrývalo všechny odborné oblasti účastníků, bylo ale zastoupeno již v Saském konsorciu. Sage Publications sice nehrálo v Saském konsorciu žádnou roli, nedokázalo však pokrýt všechny požadované tématické oblasti - předevších technické obory, čímž také nepřicházelo v úvahu.

Taylor \& Francis s Imprints (původně samostatnými nakladatelstvími, které byly začleněny do skupiny Taylor \& Francis) CRC, Routledge, Earthcan, Ashgate, Acumen, Pickering \& Chatto, Garland, Europa, Psychology Press a Spon velmi dobře pokrývalo odborné profily zúčastněných projektových partnerů - technika, př́rodní vědy, humanitní vědy, sociální vědy, psychologie, stavebnictví 
- a tím i informační potřebu projektových partnerů. Skupina Taylor \& Francis nebyla navíc zastoupena v Saském konsorciu. Čeští projektoví partneři z projektových prostředků zakoupili databázi časopisů od Taylor \& Francis.

Po dlouhém výběrovém procesu a mnoha projektových diskuzí se projektoví partneři rozhodli pro nabídku nakladatelství Taylor \& Francis, kterou zprostředkovala firma Missing Link. To umožnilo vytvořit první česko-saské konsorcium.

Po dobu trvání projektu měli projektoví partneři př́stup k rozsáhlé databázi s cca. 5000 elektronickými knihami nakladatelství Taylor \& Francis v hodnotě více než 670000 EUR. Po skončení projektu budou z fondu vybrány knihy v hodnotě 160000 EUR, které budou k dispozici všem projektovým knihovnám. Tento postup je v knihovnictví označován jako evidence based selection, krátce EBS.

Použité finanční prostředky tak budou efektivně využity a zajistí udržitelnost projektu.

\subsection{Meziknihovní výpůjční služba a semestrální aparáty}

V česko-saském prostoru učení hrají meziknihovní výpujčky tištěných médií i nadále důležitou roli. Zatímco se v prvních projektech přes meziknihovní výpůjční službu vyměňovala výhradně média z Česko-saské odborné knihovny, byl v projektu Prostor učení tento servis rozširren na všechna média zúčastněných knihoven. Mezi projektovými partnery byla uzavřena dohoda, ve které se stanovila následující pravidla:

\section{Pravidla výpůjčky}

\section{Fond Česko-saské odborné knihovny}

Výpůjční doba je minimálně jeden měsíc, maximálně však jeden rok. Výpưjční doba delší než jeden měsíc se zkrátí, vyžádá-li si médium jiný zájemce. V tomto prípadě se musí dané médium okamžitě vrátit. Po uplynutí maximální roční lhůty si může uživatel médium výpůjčit znova. Za tímto účelem musí uživatel médium předložit v domovské knihovně. Po potvrzení danou univerzitní knihovnou je v Univerzitní knihovně Chemnitz výpůjčka obnovena. 
Fond Univerzitní knihovny Technické univerzity Chemnitz (mimo Česko-saské odborné knihovny), Vysokoškolské knihovny Zittau/ Görlitz, Univerzitní knihovny ZČU PIzeň, Krajské vědecké knihovny v Liberci a Univerzitní knihovny Technické univerzity v Liberci

Doba výpůjčky činí jeden měsíc s možností dvakrát prodloužit. 0 prodloužení se však musí zažádat. Další pravidla stanovuje vždy daná univerzitní knihovna.

\section{Kopie}

Kopie odstavců z časopisů, kapitol knih nebo sbírek jsou poskytovány $v$ tištěné formě.

Díky těmto pravidlům a zásadám prímého zabezpečování se knihovny odlišují od národních předpisů v Německu i v Česku.

V České republice platí princip regionality a reciprocity. Podmínky pro meziknihovní výpůjčky si stanovuje každá knihovna zvlášt. Do systému meziknihovní výpůjční služby je zapojeno Národní centrum (národní knihovna), regionální centra (krajské knihovny), knihovny s regionálním rozsahem a specializované knihovny. Mezinárodní meziknihovní výpůjčka je možná u 6 knihoven. Před vlastním vyžádáním výpůjčky lze zadat vyhledávání v českém Souborném katalogu, jehož fond tvoří 6850000 titulů.

Od roku 2016 je oficiálně spuštěn Centrální portál knihoven (www.knihovny.cz), který umožňuje prohledávat katalogy zapojených knihoven z jediného vyhledávacího řádku (rozhraní). v souborném katalogu všech českých knihoven. Mimoto vyvíjí Národní technická knihovna portál Získej. Tato platorma bude k dispozici pro meziknihovní výpůjční službu a dodávání dokumentů.

V Německu je meziknihovní výpůjční služba regulována výpůjčním řádem. Také zde platí princip regionality tzn. knihovny by při vyřizování objednávek měly nejprve vyčerpat možnosti ve vlastním spádovém regionu.

Projektová meziknihovní výpůjční služba se využívá také pro vytváření tištěných semestrálních aparátů. Pod pojmem semestrální aparát se rozumí sbírka médií - v prípadě projektu Prostor učení se jedná o tištěné knihy - která je po dobu celého semestru k dispozici přímo v knihovně. Tyto semestrální aparáty obsahují povinnou literaturu k určitému semináři. Zatímco jsou semestrální aparáty na německých vysokých školách velmi oblíbené, nehrají na českých 
vysokých školách přiliš velkou roli a využivány jsou jen výjimečně. Prof. Dr. Elke Mehnert, profesorka pro německou literaturu na Západočeské univerzitě $\checkmark$ Plzni tyto aparáty zavedla pro své studenty a studentky a využívala pro ně převážně knihy z Univertzitní knihovny Chemnitz.

Milník 1 Konsorciální pořizení informačních zdrojü/ meziknihovní výpưjční služba/semestrální aparáty predstavoval nejdůležitější obsah projektu Prostor učení. Byly dosaženy všechny cíle, především vytvoření prvního česko-saského konsorcia pro pořizení elektronických médií, které zajistilo udržitelnost projektu i po jeho skončení a působí jako vzor pro další knihovny. 



\section{MEDIÁLNÍ TECHNIKA A INFRASTRUKTURA V PROPOJENÉM PROSTORU UČENÍ}

Falk Maiwald 


\subsection{Nový pohled na prostory učení vědeckých knihoven}

Díky "Shift from Teaching to Learning" se rozvoj kompetencí v dnešní době dostává do centra zájmu vzdělávacích aktivit studentů. Ke klasické prezenční výuce v přednáškových sálech nebo seminárních místnostech pribyly místně a časově flexibilní formy učení - individuální i skupinové. Pro zajištění potřebných kompetencí svých absolventů a absolventek, dosažení pozitivní image a tím posílení konkurenceschopnosti prii získávání nových studentů musí vysoké školy vytvořit pro své studenty nové rozšřr̃ené rámcové podmínky.

Jednou z možností, jak toho dosáhnout, je „tř̌ní” vytvárení prostorů učení, především v podobě knihoven. Prostory učení knihoven resp. v knihovnách Ize chápat jako virtuální místa střetu nabídky a poptávky. Na straně nabídky je jejich realizace zásadně ovlivněna mimo jiné stále rozvinutěǰsími informačními a mediálními technologiemi. Následkem toho je urychlován i další vývoj nabídky digitalizace ve výuce (e-learning, platformy pro řízení výuky), ve spolupráci (webové konference, nástroje pro spolupráci), digitalizace médií (e-časopisy, e-knihy, databáze) a další. Na straně poptávky pak stojí studenti tzn. „internetové generace“: mají zkušenosti s médii, umí zacházet s výpočetní technikou, čímž sami o sobě kladou vysoké nároky na mediální a IT strukturu knihovny, kterou chtěji využívat jako reálné, lokální komunikační centrum a zároveň místo pro učení. $V$ neposlední řadě je třeba zohlednit také požadavky vyučujících a výzkumných pracovníků, kteři chtěji v lokálním prostoru učení efektivně provádět rešerše a dalši činnosti.

Vědecké knihovny se u „tržního" technického vybavení prostorů učení zaměřily na požadavky interních skupin uživatelů, modernizovaly svou lokální IT infrastrukturu pro individuální pracovní místa i skupinové místnosti. Podobné lokální nabídky a infrastruktura jsou již řadu let k dispozici i pro externí uživatele. Projektoví partneři tak otevírají své dveře i studentům, vyučujícím a výzkumných pracovníkům z jiných organizací - dokonce i z dané sousední země. Je proto důležité v prípadě potřeby prìzpůsobit lokální postor učení specifickým požadavkům, a pomocí informačního vzdělávání (viz kapitola 5) zajistit, aby externí uživatelé mohli danou nabídku efektivně využívat.

Při orientaci na uživatele však zůstal jeden aspekt - bezpochyby nejen u projektových knihoven - dosud mimo hlavní pozornost. Na „trhu” vědeckých knihoven Ize identifikovat takové skupiny uživatelů, které mají úzký vztah nebo interakci s jinými knihovnami, vysokými školami nebo univerzitami. Jinými 
slovy existují interní a externí skupiny uživatelů se společnými úkoly a zájmy. Může se jednat např. o projektové studie nebo výzkumné semináře skupin studentů, výzkumné projekty vědeckých pracovníků nebo výměnu zkušeností a znalostí mezi vyučujícími různých univerzit a vysokých škol. Zaměstnanci knihoven se s bilaterálními nebo multilaterálními přístupy setkávají zejména prì odborné výměně informací nebo projektech, jako je tento. U klasických forem se scházejí fyzicky v reálných, lokálních pracovních a učebních prostorech.

V dnešní době je s pomocí internetových technologií možné vytvářet virtuální studijní a pracovní prostředí jako místa sociálních interakcí a přitom (zpravidla) snadno překonávat politické a geografické hranice. Prakticky tak mohou tyto virtuální prostory pro učení a práci využívat aktéři z celého světa. Hlavními výhodami jsou:

- vysoká časová flexibilita,

- nízký práh pro výměnu a spolupráci a tím (v ideálním případě) zlepšení výsledků projektu,

- pozitivní vliv na image knihovny v kontextu digitalizace a

- nízká časová a finanční náročnost díky „ušetřenému“ cestování.

Přesně na tomto místě navázaly první úvahy k projektu. Projektoví partneři tehdy vyvinuli první všeobecné zadání pro vytvoření společného přeshraničního virtuálního a pracovního prostoru, který byl později označen jako propojený prostor učení, a zahrnuli ho do milníku Technické vybavení prostoru učení.

Virtuální propojený prostor učení může být - podobně jako online community - chápán jako neformální spojení studentů, výzkumných pracovníků a vyučujících, kteří na základě společných zájmů nebo problémů spolu přes internet komunikují, spolupracují, vyměňují si znalosti a zkušenosti a přitom se od sebe navzájem učíí. K tomu využívají informační a mediální techniku i další infrastrukturu knihovny. V popředí projektového milníku stál vývoj, testování a implementace propojených subprostorů učení mezi projektovými partnery. Hlavní snahou bylo dosáhnout tak společného cíle a v rámci vzájemného propojení a spolupráce posílit vědecké struktury v regionu a podpořit individuální přeshraniční učební a výzkumné procesy. Jako vedlejší efekt mohly knihovny upevnit resp. zlepšit svůj image moderního mediálního centra a místa učení.

16 Kupfer (2015), str. 204. 
Klíčovým úkolem milníku bylo naplánovat a nakoupit odpovídající technické vybavení resp. potřebnou infrastrukturu prostoru učení pro projektové partnery. Zohledněním požadavků na lokální prostory učení jednotlivých projektových partnerů se podpořila tvorba synergií a ekonomicky udržitelné využití. Výchozím krokem pro technické plánování a specifikaci byl vývoj scénářu využití zaměřených na skutečné potřeby v zamýšleném prostoru učení (srov. kapitola 3.2.). Za tím účelem byla provedena simulace možných konstalací meziorganizační resp. přeshraniční spolupráce projektových partnerů v rámci webových konferencích, multimediálních prezentací apod. Z těchto scénářu pak partneři odvodili potřebnou technickou infrastrukturu, specifikovali technické parametry a požadavky a konečně pořídili a implementovali výpočetní a mediální techniku (kapitola 3.3.).

Proto, aby technická inovace mohla být otestována a později efektivně využívána v běžné praxi, je nezbytné, aby ji potencionální uživatelé akceptovali a prakticky vyzkoušeli. Za tímto účelem byly v milníku naplánovány a uskutečněny různé společné aktivity, především iniciační workshop v Plzni v lednu 2017 nebo praktická stáž v Liberci-Görlitz v květnu 2017 (viz kapitola 3.4). Projektoví partneři uchopili ale i další přiležitosti k využití a prezentaci techniky v lokálních prostorech učení a v propojeném prostoru učení. Za zmíňku stojí konsekventní nasazení techniky při měsíčních webových konferencích projektových partnerů nebo velmi úspěšný virtuální seminář na téma informační vzdělávání v dubnu 2018. Vedle toho dostaly prostor i další technické možnosti, které smysluplně podpořily práci na projektu. Jako př́klad jmenujme dvojjazyčné návody k použití vyvinuté v rámci projektu.

\subsection{Základní scénáře využití pro nový propojený prostor učení}

Ve zde definovaném prostoru učení probíhá komunikace resp. spolupráce v užším smyslu v reálném čase. To znamená, že skupiny uživatelů mezi knihovnami spolu v rámci webových konferencí a pomocí interaktivních nástrojů pro spolupráci živě diskutují, pracují na projektech a vyvíjí koncepty, jejichž výsledky se zpracovávají elektronickou cestou. V širším slova smyslu ale propojený prostor učení zahrnuje také časově oddělenou komunikaci a spolupráci. Ta může probíhat bud'v následném pořadí nebo obousměrně, např. přes nástroje projektového managementu, dokumentační, e-learninové systémy, systémy managementu znalostí nebo centrální datové paměti k zálohování dat. 
V popředí tohoto projektového milníku stál - jak už bylo popsáno v kapitole 3.1. - vývoj možných scénářů využití živé komunikace se zohledněním techniky lokálních prostorů učení.

\section{Využití pro zaměstnance knihovny}

Knihovny mohou využít možnosti propojeného prostoru učení pro své vlastní účely. Skupina knihovníků může napríklad nad rámec státní hranice nebo sídla knihovny pořádat workshopy nebo školení k pořizování elektronických médií nebo si vyměňovat informace k tématu informačního vzdělávání. Vedení kooperujících knihoven může zase vést interaktivní webové konference ke strategickém vývoji a společným aktivitám.

Dále jsou možné např. školení, porady a workshopy pro studenty, vyučující a výzkumné pracovníky, kteří se nemohou zúčastnit prímo na místě, jako např. zahraniční uživatelé.

\section{Využití pro studenty, výzkumné pracovníky, vyučující}

Vyučující a výzkumní pracovníci si mohou knihovnu resp. její vybavení zamluvit a využívat jako propojený prostor pro učení a práci. Možné jsou také nabídky pro odborné kolegy ke spolupráci na výzkumných tématech nebo prezentace před firmami resp. v sousední zemi, virtuální workshopy, nebo poradenství pro grémia. Za zdůraznění stojí především interakce přesahující rámec běžné webové konference, např. současná práce na jediném, společném dokumentuy ze dvou nebo více pracovišt. Přínosné je také využití pro studenty a jejich vedoucí vědeckých nebo závěrečných prací, a to především v prípadech, kdy je nutná konzultace $\mathrm{k}$ určitému tématu se vzdálenou národní nebo mezinárodní firmou. Vyučující nebo výzkumníci se ale mohou do knihovny „uchýlit“ jen proto, že jsou pro ně prostorové podmínky v knihovně zkrátka výhodnější.

Tyto scénáře Ize pomocí dalších príkladů a nápadů teoreticky ještě rozvinout a rozšririt. Zmíněné i další praktické možnosti využití byly v průběhu projektu nejednou názorně prezentovány resp. prakticky využity (viz kapitola 3.4). Zde představené koncepty jsou však pro následné odvození nejdůležitějších potřeb a požadavků na technické vybavení projektových partnerů dostačující. 


\subsection{Požadavky, pořízení a implementace nové mediální a výpočetní techniky}

Projektoví partneři odvodili z výše představených scénářu infrastrukturu a př́stroje nezbytné pro technické vybavení projektu. Na obrázku 1 je znázorněn princip živé komunikace přes internet v propojeném prostoru učení i nejdůležitější technické prístroje.

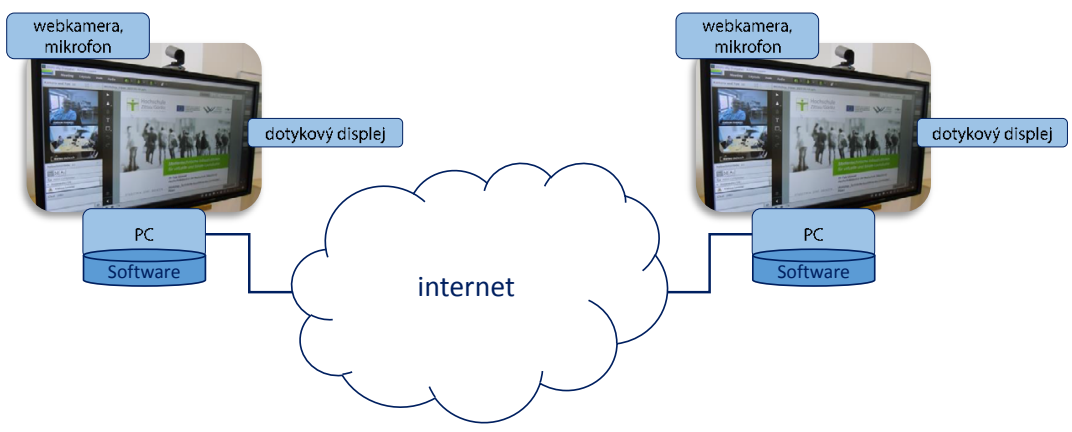

Obrázek 1. Princip systému živé komunikace v propojeném prostoru učení. Zdroj: vlastní zpracování; Foto: VŠK Zittau/Görlitz.

\section{Přístup k internetu - hlavní piliř infrastruktury propojující organizace}

Podmínkou proto, aby komunikace mohla probíhat nad rámec státní hranice resp. mezi pracovišti, je vysokorychlostní internet a dodatečný speciální software (funkce pro videokonference, spolupráci pomocí živého přenosu apod.). Tyto předpoklady byly u projektových knihoven resp. díky př́islušným výpočetním střediskům vysokých škol a univerzit splněny. V Německu jsou státní vysoké školy připojeny k „velmi rychlému“ internetu přes Německou výzkumnou sít (Deutsches Forschungsnetz - DFN) ${ }^{17}$, v České republice pak přes Czech Education and Scientific NETwork (CESNET) ${ }^{18}$. Krajská vědecká knihovna v Liberci disponuje přístupem k vysokorychlostní síti přes Liberecká IS. V propojeném prostoru učení představuje přístup k internetu jeden z hlavních piliřu infrastruktury spojující jednotlivá pracoviště a organizace.

17 DFN je provozován Spolkem na podporu Německé výzkumné sítě (Verein zur Förderung eines Deutschen Forschungsnetzes e.V.), srov. DFN (2018).

${ }^{18}$ CESNET je provozován sdružením CESNET, z. s. p. 0. , srov. CESNET (2018) https://www.cesnet.cz/. 


\section{Konferenční nástroje - software pro komunikaci a interakci}

Pro realizaci online akcí byl k dispozici nejprve software Adobe Connect. Ten je v Německu pro všechny vysoké školy licencován přes DFN, v Čechách pak přes CESNET. V průběhu projektu se objevila další možnost v podobě nástroje WebEx společnosti Cisco, který však měla licencovaný jen Technická univerzity Chemnitz. Oba nástroje jsou si velmi podobné; kvalita přenosu se ale zdála lepší u aplikace WebEx. Společným znakem obou nástrojů je, že stačí pouze jedna licence resp. jeden vlastník licence, který může k živé komunikaci na online akce přizvat další partnery. Ve scénáři ke spolupráci mezi knihovnami tento fakt byl a stále je relevantní především pro Krajskou vědeckou knihovnu v Liberci, která nevlastní žádnou odpovídající licenci. Této výhody mohou využít např́klad také firmy. Pro projekty a porady s vyučujícími a výzkumníky se mohou i ony přihlásit do propojeného prostoru učení.

\section{Počítačová a mediální technika - lokální hardware}

Vědecké knihovny v lokálním prostoru učení jsou moderní počítačovou a mediální technikou již víceméně vybaveny. Kabiny pro individuální práci pravidelně disponují IT technikou (klasicky: PC s monitorem, př́p. scanner). V trendu BYOD ${ }^{19}$ si uživatelé nosí svá vlastní koncová zařízení, které využívají jak v pracovních kabinách, tak na pracovních místech ve volném prostoru bud' pro samostatnou práci nebo krátké rešerše. Podmínkou je výkonný WLAN a prípojky do elektrické sítě.

Skupinové práce, které jsou pro prostor učení především zajímavé, probíhají v rámci knihovny $v$ místnostech nebo vymezených prostorech vybavených prímo pro tento účel. Běžné jsou jak pevně zabudované počítače, tak stále častěji i infrastruktury BYOD. Jako mediální technika se většinou využívají beamery a audiozařízení. V souvislosti s digitalizací nastupují na místo klasických tabulí, flipchartů nebo beamerů stále častěji interaktivní tabule. Podskupinou interaktivních tabulí jsou prezentační a interaktivní displeje, tzv. dotykové displeje vybavené vlastní počítačovou a sítovou technikou. Pro prezentační účely je Ize ovládat i pomocí osobních počítačů, laptopů nebo

19 „Bring Your Own Device“ popisuje přístup k IT infrastruktuře dané organizace pomocí externích přístrojů, srov. DINI (2013), str. 65. Takové přístroje jsou především notebooky, tablety a smartphony. 
mobilních prístrojů s funkcí Miracast ${ }^{20}$.

V zásadě je pro živou komunikaci přes internet resp. online akce zapotřebí alespoň jeden počítač/laptop s bezdrátovým připojením (WLAN), monitor a/nebo beamer, webkamera a mikrofon. Pro interaktivní skupinovou práci se namísto klasických monitorů nebo beamerů dobře hodí dotykové displeje. Velmi velké monitory (65" a více) jsou umístěny v odpovídajících místnostech pro skupinovou práci. V ideálním případě je "technické portfolio“ doplněno ještě o webovou kameru s vysokým rozlišením a kvalitní konferenční mikrofon, určenými speciálně pro podobné prostory.

\section{Výběr a pořizení technického vybavení}

Hlavní výzvou pro projekt bylo zkombinovat požadavky uživatelů na vybavení lokálních prostorů učení (viz kapitola 3.1) a požadavky na propojený prostor učení. Na základě scénářů popsaných v kapitole 3.2. se tři projektoví partneři rozhodli pro nákup dotykových displejů s adekvátní konferenční technikou. Pro dosažení cíle projektu pořídili i oba další projektoví partneři počítačovou a mediální techniku.

Specifikace technických požadavků byla možná jen díky rozsáhlým rešerším na trhu a řadě konzultací se specialisty. Ukázalo se, že vyvinuté scénáře byly pro pochopení nabízené techniky vzhledem k hlavní myšlence prostoru učení prínosné. Rozhodujícím aspektem u displejů byla jejich mobilita, aby je bylo $\checkmark$ prípadě potřeby možné použít v různých místnostech. Poté následovalo běžné výběrové řízení, zadání zakázky resp. pořizovací proces přes příslušnou centrální organizaci projektových partnerů.

Lead partner, Univerzitní knihovna Technické univerzity Chemnitz, pořídil dotykový displej Galneoscreen 65" s nastavitelným podstavcem a sklápěcí stůl pro laptop/klávesnici tzv. Swift-Turn-Table. Díky svým rozměrům a mobilitě mǔže být displej později využit pro další projektové záměry i v nově vznikající budově knihovny.

Vysokoškolská knihovna Zittau/Görlitz (VŠK) zakoupila pro své dvě pobočky vždy jeden dotykový displej Promethean ActivPanel, 65" s nastavitelným podstavcem, zabudovaným počítačem a Extron Sharlink k lokálnímu standardu Miracast, určenému až pro 4 uživatele (viz obrázek 2). V podobě Logitech

${ }_{20}$ Miracast je standard umožňující přenos obsahů z monitorů na smartphonech nebo tabletech na větší displeje. 


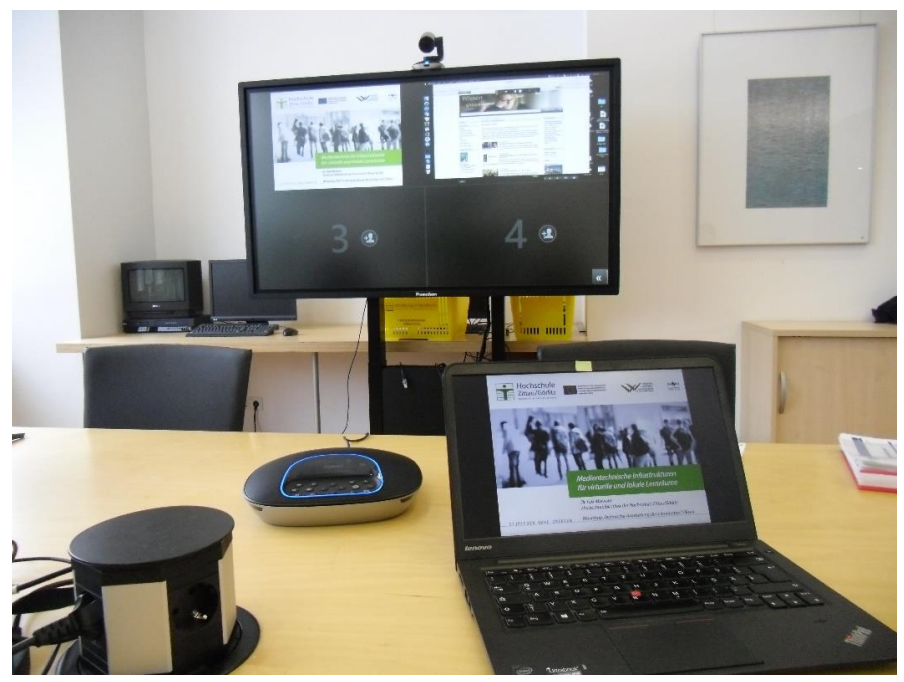

Obrázek 2. Př́klad pro Miracast s laptopem a aktivním panelem ve skupinové místnosti. Foto: VŠKK Zittau/Görlitz.

ConferenceCam Kit navíc přibyla velmi výkonná USB webkamera a konferenční mikrofon. Všechny přístroje jsou k dispozici ve velké skupinové místnosti.

Univerzitní knihovna Západočeské univerzity v Plzni zakoupila z projektových prostředků šest notebooků HP ProBook 640 G2 a jeden beamer Epson EB-535W+Epson Wall Mount ELPMB45. Tuto techniku využívá jak pro školení a semináře pro uživatele v lokálním prostoru učení, tak při projektových seminárích, workshopech a webových konferencích.

Krajská vědecká knihovna v Liberci vybavila svůj velký konferenční sál výkonným projektorem a promítacím plátnem pro prezentace $v$ rámci přednášek, konferencí nebo pro filmovou projekci. Dále nechala nainstalovat speciální kameru vybavenou softwarem umožňujícím nejen nahrávky v prostoru, ale také přenos do virtuálního prostředí např. na YouTube.

Univerzitní knihovna Technické univerzity v Liberci pořídila dva laptopy a jeden mobilní beamer, který se v případě potřeby využije v lokálním prostoru učení. Stejně jako lead partner a VŠK Zittau/Görlitz zakoupila i Univerzitní knihovna v Liberci interaktivní dotykový displej (ActivBoard) s podstavcem včetně adekvátního vybavení pro videokonference, napr. bezdrátový mikrofon a full HD kameru. 


\section{Další nástroje v propojeném prostoru učení}

Jak bylo popsáno v kapitole 3.2., Ize v propojeném prostoru učení využívat také nástroje pro časově oddělenou komunikaci resp. spolupráci, např. speciální e-learningové platformy jako systém Moodle (viz kapitola 5) und nástroje k ukládání projektových dokumentů.

Již na začátku projektu se projektoví partneři shodli na tom, že práce na projektu musí být pro všechny partnery transparentní a jasně dokumentována. Dokumenty a soubory by proto měly být v elektronické podobě uloženy na schráněném místě přístupném pro všechny partnery. $V$ době, kdy nejrůznější poskytovatelé nabízejí na internetu cloudová úložiště, je centrální úložišš̌ pro dokumenty resp. soubory snadno realizovatelné. S ohledem na bezpečnostní aspekty je ale třeba vnímat takové nabídky kriticky. Úkolem tedy bylo vytvořit vlastní, bezpečný projektový cloud. Díky odborné kompetenci a odpovídajícím zdrojüm výpočetního centra Vysoké školy Zittau/Görlitz ( $\mathrm{k}$ roli výpočetních center viz kapitola 3.5 ) bylo implementováno spolehlivé řešení - software Filr firmy Micro Focus. Všichni zaměstnanci projektu měli permanentní, individuální př́stupová práva na cloud projektu a všechny obsahy přes rozhraní Filr Web (pro PC/laptop přes program Filr a pro mobilní př́stroje přes aplikaci Filr App).

\subsection{Využití techniky v přeshraničním propojeném prostoru učení}

Řada aktivit, jako prezentace technických možností, školení, vývoj srozumitelných návodů k použití a v neposlední řadě konsekventní zapojení do práce na projektu byly klíčem pro úspěšnou implementaci a využití pořizené techniky a softwarových nástrojů.

\section{Workshop v Plzni - počátek technického prostoru učení}

V rámci workshopu Technické vybavení prostoru učení v lednu 2017 v Plzni představili všichni projektoví partneři své lokální technické a infrastrukturní podmínky. S cílem zdůraznit nejprve všem zúčastněným zaměstnancům projektu výhody využívání technického vybavení, představila VŠK Zittau/Görlitz $\checkmark$ úvodu různé možnosti jeho využití. $V$ rámci živé prezentace mezi Plzní a Zittau byla názorně předvedena výkonnost konferenčního softwaru a konferenční 
techniky, včetně již zapojených dotykových displejů a periférní konferenční techniky v Zittau.

\section{Výměnná stáž Liberec/Görlitz - hlavní téma: modul IK a webová konference se všemi partnery}

Obě projektové knihovny v Liberci a Vysokoškolská knihovna se sídlem v Görlitz uskutečnily výměnnou stáž v rámci milníku v květnu 2017. Hlavním bodem těchto projektových dnů byla otázka, jak bude modul informačního vzdělávání obsahově koncipován a technicky resp. mediálně podložen. $V$ této souvislosti byly diskutovány aspekty e-learningu a otázky informačního vzdělávání (k tomuto milníku viz kapitola 5).

Pořízená počítačová a mediální technika byla zapojena jak při lokální skupinové práci zaměstnanců projektu, tak při ca. hodinové závěrečné prezentaci, která byla př́mo z Görlitz jako webová konference sdílena se všemi ostatními projektovými pracovišti. Přitom se jasně ukázaly výhody tohoto řešení pro virtuální spolupráci mezi knihovnami. Vedle samotných účastníků týdenní stáže se mohli na prezentaci podílet i zaměstnanci, vedení a další hosté ze vzdálených pracovišt́t.

\section{Pravidelné online porady - nový prístup k práci na projektu}

Z pozitivních zkušeností v rámci výměnné stáže se vyvinul nový, pravidelný způsob pro konzultace a koordinaci práce na projektu. Od 12. června 2017 se začaly konat měsíční online porady všech projektových partnerů. Díky zakoupené technice a nástroji Adobe Connect - později WebEx - bylo možné zodpovídat otázky k projektu a diskutovat o konkrétních úkolech. Tento způsob nebyl jen velmi efektivní, ale díky své pravidelnosti zlepšil i angažovanost projektových partnerů. Do června 2018 se uskutečnilo dvanáct online porad.

\section{Lokální využití techniky}

Jak již bylo představeno, byla v rámci „projektové společnosti“ uskutečněna řada kroků pro efektivní využití techniky pro práci na projektu i pro práci zúčastněných knihoven včetně odborné výměny. Jako příklad bychom neměli zapomenout zmínit, že Krajská vědecká knihovna v Liberci plánuje přenos závěrečné konference projektu přes YouTube. 
Partneři se zabývali také scénáři využití pro další skupiny uživatelů např. studenty, vyučující nebo výzkumné pracovníky (více v kapitole 3.2), přičemž se zaměřili především na lokální využití techniky. Aby uživatelé knihovny mohli pochopit a akceptovat nové technické vybavení, bylo nezbytné vyškolit v práci s novou technikou nejprve vlastní zaměstnance knihovny. Někteří projektoví partneři proto vytvořili pro své uživatele tištěné tutoriály v českém a německém jazyce, např. s funkcemi a možnostmi ovládání dotykových displejů.

Úspěch se postupně dostavil. Všichni projektoví partneři se shodli, že zakoupená technika byla studenty velmi dobře príijata a je často využívána. Na interaktivní tabuli ActiveBoard na Technické univerzitě v Liberci jsou pro studium/výuku k dispozici různé interaktivní aplikace a výuková videa a pobyt v prostorách knihovny zpř́ijemňuje relaxační hudba a reklamní videa.

\section{Virtuální seminár - akvizice nových skupin uživatelů}

Interaktivní meziorganizační prostory pro učení a práci musely pro nové skupiny uživatelů - stejně jako předtím pro vlastní zaměstnance projektu a knihovny - nejprve získat viditelnou a praktickou podobu. Za tímto účelem uskutečnili projektoví partneři v dubnu 2018 pod záštitou VŠK Zittau/Görlitz virtuální seminář, na který do své „domovské knihovny“ pozvali (odpovídající reklamou) vyučující, studenty a výzkumné pracovníky. S nasazením potřebné techniky a přes WebEx tak proběhla další webová konference mezi pracovišti v Chemnitz, Plzni, Liberci, Zittau a Görlitz. V publiku byly ve velkém počtu zastoupeni účastníci ze všech relevantních skupin uživatelů.

Zvláštností tohoto semináře bylo, že se publikum seznámilo s výsledky tohoto i dalších milníků včetně celého spektra úspěchů projektu Prostor učení - Knihovnická informační platforma. Partneři jeden po druhém představili možnosti využití médií a meziknihovní výpůjční služby a prezentovali elektronické knihy zakoupené v rámci konsorcia. Na konci semináře byly na dotykovém displeji „živě” předvedeny možnosti interakce při skupinové práci mezi jednotlivými pracovišti.

Pozitivní feedback k této akci jasně ukázal, že výsledky projektu mohou být relevantní a užitečné pro celou řadu uživatelů. 


\subsection{Lessons learned}

V předchozích kapitolách již byly popsány důležité faktory pro úspěch plánování, nákupu a využití techniky.

Dalším významným faktorem úspěchu byla podpora ze strany výpočetních center resp. oddělení IT u projektových partnerů. Odborné posudky byly pro plánování, zapojení a provoz mediální, výpočetní techniky a odpovídající infrastruktury nepostradatelné. Odborníkům z oblasti mediální techniky a IT partnerských knihoven byla přisouzena rozhodující role při transformaci návrhů k technickému vybavení i jeho využití do účelných konceptů a jejich následné realizaci. Byly naplánovány a porovnány různé konfigurační varianty. Ohledně plánovaných investic probíhala mezi projektovými partnery pravidelná, intenzivní komunikace. V souvislosti se zř́zením virtuálních pracovišt byla provedena řada testů. Pomoc IT expertů byla nezbytná také při první implementaci nové techniky v reálném provozu.

Závěrem Ize konstatovat, že pořízení a využití počitačové a mediální techniky posunulo vzájemnou práci projektových knihoven a spektrum nabídky pro uživatele knihovny na úroveň, která plně odpovídá trendu digitalizace a dalšímu vývoji v této oblasti.

\section{Použitá literatura}

Kupfer, F.: Lernen und Arbeiten in Lernort übergreifenden Netzwerk, in: Wittwer, W.; Diettrich, A.; Walber, M. (Hrsg.): Lernräume, Wiesbaden 2015, str. 204-217.

DINI, Deutsche Initiative für Netzwerkinformation e.V.: Die Hochschule zum Lernraum entwickeln, Kassel 2013.

DFN, Verein zur Förderung eines Deutschen Forschungsnetzes e.V., URL: https://www.dfn.de/xwin/ , poslední př́stup 16.08.2018.

CESNET, z. s. p. o., Czech Education and Scientific NETwork, URL: https://www.cesnet.cz/, poslední prístup 16.08.2018. 



\section{OPEN ACCESS}

Zdeňka Firstová

Miroslava Pourová 


\subsection{Základy Open Access}

Každá vědecká práce začíná studiem odborné literatury, především článků v recenzovaných vědeckých časopisech. Paradoxem je, že ačkoliv počet publikací stoupá, jejich dostupnost se zásluhou stále rostoucích cen časopisư snižuje. Nejvíc tato skutečnost doléhá právě na vědce, kteří jsou nuceni, vzhledem ke způsobu hodnocení vědy, v časopisech publikovat. Velcí komerční nakladatelé vydávající prestižní časopisy zvyšují ceny předplatného časopisů a databází, které tituly zpřístupňují. Autoři článků, které jsou publikovány bez nároku na honorár̆, se tak mnohdy nedostanou ani k pracím svých kolegů, protože si jejich instituce nemůže dovolit nakoupit veškeré potřebné časopisy. Alarmující je, že už se to týká i nejbohatších univerzit. Již v roce 2012 upozorňoval ředitel knihovny Harvardské univerzity Robert Darnton na paradoxní situaci: členové akademické obce provádí výzkum, zdarma o něm píší, recenzují práce svých kolegů, pracují v edičních radách a pak si kupují zpět výsledky své práce za přemrštěné ceny. ${ }^{21}$

Dalším paradoxem je, že věda a výzkum jsou z velké části financovány z veřejných peněz, ale jejich výsledky jsou publikovány v komerčních časopisech, a prístup k nim je opět placen z veřejných zdrojů. Někteří akademici proto začali v souvislosti s publikováním hovořit o krizi tradičních vědeckých časopisů. Kromě problému s cenou a dostupností poukazuje řada autorů na časovou prodlevu. Mezi sepsáním článku a jeho vydáním v klasickém časopise uběhne dlouhá doba. Zejména u prestižních titulů zpoždění způsobuje nejen dlouhé recenzní řízení, ale zejména převis nabízených prací, takže pro některé obory už nejsou takové časopisy zdrojem nových informací.

Iniciativa Open Access (OA), prosazující myšlenku, že by výsledky vědy a výzkumu měly být př́istupné okamžitě a zdarma, se snaží tyto rozpory odstranit.

Principy Open Access byly formulovány počátkem tohoto století třemi iniciativami: Budapest Open Access Initiative (únor 2002), Bethesda Statement on Open Access Publishing (červen 2003) a Berlin Declaration on Open Access to Knowledge in the Sciences and Humanities (říjen 2003), souhrnně nazývanými BBB-iniciativy. Stručně řečeno, Open Access (otevřený př́stup) zaručuje vědecké veřejnosti bezplatný a trvalý př́istup k odborným textům na internetu. Tím odstraňuje nerovnost $v$ přístupu $\mathrm{k}$ informacím a zásadně mění model

${ }^{21}$ https://www.theguardian.com/science/2012/apr/24/harvard-university-journal-publishersprices 


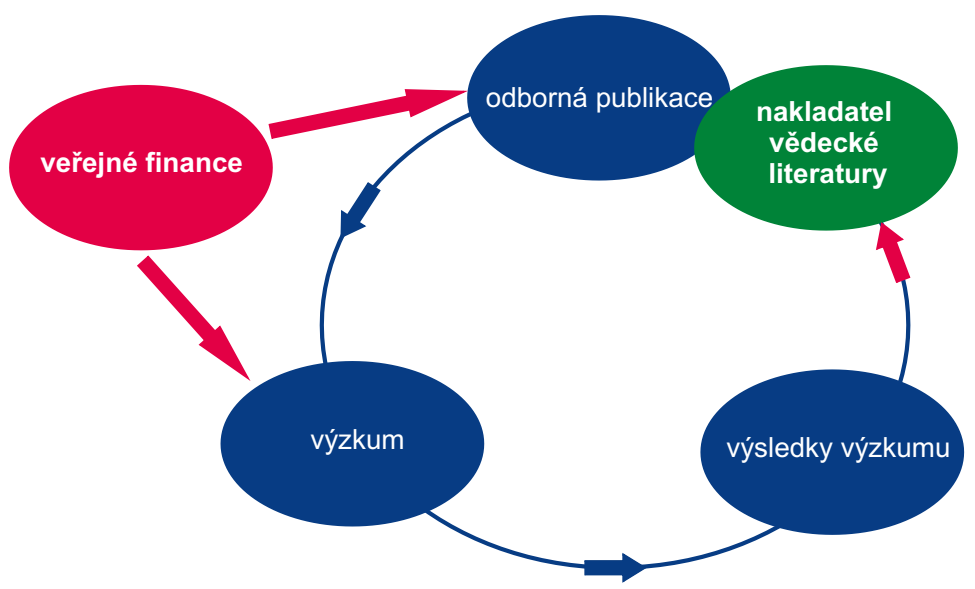

Obrázek 1. Vědecká práce.

financování vědeckého publikování. Texty zveřejněné v režimu $O A$ je možné bez zvláštních právních, finančních nebo jiných omezení dále kopírovat, reprodukovat, tisknout, rozmnožovat a prohledávat. Iniciativa je zároveň reakcí na výrazný vliv internetu v oblasti šřrení informací a vědeckých poznatků a poskytuje alternativu ke klasickému modelu publikování u komerčních nakladatelů.

Jednou z možností, jak zajistit otevřený přístup k publikacím je tzv. zlatá cesta, kdy otevřený přístup zajištuje vydavatel. Způsob, jakým to umožňuje, je různý. Existují $\mathrm{OA}$ časopisy, které jsou plně otevřené, tj. celý jejich obsah je okamžitě přístupný komukoliv zdarma. Časopisy jsou bud' financovány nadacemi či organizacemi (velmi často jsou to univerzity) a publikování je v nich bud' zdarma, nebo je za uveřejnění článku potřeba zaplatit poplatek APC (article publishing charge nebo jinde nazývaný article processing charge). Seznam 11,5 tisíce Open Access časopisů Ize nalézt v adresári DOAJ - Directory of Open Access Journals. ${ }^{22}$

Díky tomu, že hnutí Open Access působí již téměř dvě desetiletí, ustoupila rada klasických vědeckých časopisů vytrvalému tlaku a umožňuje autorům za poplatek APC publikovat konkrétní článek v režimu otevreného přístupu. Protože jsou v těchto časopisech vedle sebe otevřené články a články přístupné pouze předplatitelům, nazýváme tyto časopisy hybridními. Kromě toho existují i časopisy, které zpřístupní články po určitém časovém embargu.

22 www.doaj.org 
Řada univerzit pro podporu zlaté cesty $\mathrm{OA}$ vyčlenila finanční prostředky ke zř́zení fondů, ze kterých jsou poplatky hrazeny. Protože finanční zdroje nejsou neomezené, většinou se fond vyčerpá, aniž by byli uspokojeni všichni zájemci. Proto jsou stanovována různá kritéria k získání príspěvku na APC, např. požadavek na určitou výši impakt faktoru časopisu, výše maximální dotace, maximálně jedna žádost pro každého autora apod.

Druhou možností otevřeného př́stupu je tzv. zelená cesta. Tuto formu zajištuje sám autor tím, že vloží práci do Open Access repozitá̌e, tj. do chráněného digitálního úložiššě, které musí zaručit trvalý prístup k vloženým publikacím. $\checkmark$ zásadě rozeznáváme repozitáre institucionální (těch je cca 85\%), které jsou budovány zpravidla univerzitami nebo jinými vědeckými pracovišti, oborové repozitáře, kde jsou zveřejňovány publikace podle obsahového zaměření, nebo repozitáře pro určitý druh dokumentu (elektronické dokumenty, digitalizované dokumenty, data).

Nejstarším a nejznámějším oborovým repozitářem je arXiv ${ }^{23}$ jehož počátky sahají do počátku 90 . let 20. století. Byl původně vytvořen $\mathrm{k}$ rychlé výměně vědeckých informací pro komunitu fyziků, dnes zahrnuje preprinty z fyziky, matematiky, počítačové vědy, kvantitativní biologie, financí a statistiky.

\section{Typy open access}

\section{zlatá cesta}

\section{otevřený přístup zajišt'uje vydavatel}

časopisy s plně otevřeným př́stupem = celý obsah je okamžitě zpřístupněn všem zdarma

open access s časovým embargem $=$ plné texty jsou zpřistupněny po uplynutí lhůty stanovené nakladatelem

hybridní časopisy = umožňují po zaplacení publikačního poplatku zprístupnění článku, jsou zde vedle sebe články

s placeným i otevřeným přistupem

\section{zelená cesta}

otevřený přístup zajišt'uje autor

autor práci publikovanou v klasickém časopise / sborníku uloži do otevřeného repozitáře

Obrázek 2. Schéma Typy OA.

${ }^{23}$ https://arxiv.org 
Seznam repozitárú s údaji o instituci provozovatele, zemi, typu repozitáře, obsahovém zaměření, typu a počtu obsažených dokumentů aj. s možností filtrování a vyhledávání poskytuje The Directory of Open Access Repositories - OpenDOAR ${ }^{24}$. Instituce se také mohou obrátit na Repositories Support Project ${ }^{25}$, kde naleznou pomoc při zakládání repozitáre.

Rostoucí význam Open Access potvrzuje i fakt, že se za posledních deset let počet OA časopisů i repozitárư zdvojnásobil. V současnosti je v DOAR evidováno přes 3,5 tisíce repozitáruu. Německo je s počtem 204 repozitárư čtvrté na světě (za USA, Velkou Británií a Japonskem). Česká republika zde má zaregistrováno 18 repozitár̆ŭ. ${ }^{26}$

Dnes již má většina renomovaných komerčních nakladatelství zformulovanou OA politiku a umožňuje autorưm ukládat nějakou formu jejich publikací do otev̌rených repozitáru. Ačkoliv jsou vědci autory článků, většina licenčních smluv s nakladateli značně omezuje jejich práva nakládání s dílem. Autor při publikování svá vlastnická práva zpravidla převádí na nakladatele. Exkluzivní právo na vydání či zpř́stupnění práce lze ošetřit dodatkem ke smlouvě o autoarchivaci ${ }^{27}$. Pro ochranu svého díla může autor zvolit tzv. otevřenou licenci Creative Commons a Science Commons. ${ }^{28}$

K usnadnění orientace autora v nakladatelské politice časopisů slouží databáze SHERPA/ROMEO, která barevně rozlišuje jednotlivé formy e-printu. ${ }^{29}$

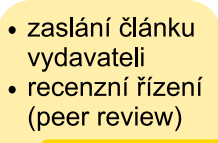

PREPRINT
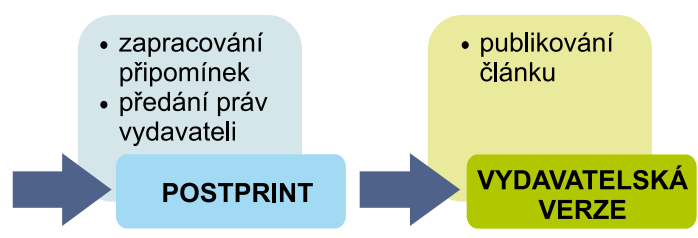

Obrázek 3. Typy e-printů.

\footnotetext{
24 www.opendoar.org

25 www.rsp.ac.uk

26 Data z 11.05.2018.

27 Vzor je dosputný na http://sparc.arl.org/.

${ }^{28}$ Všechny národní verze CC licencí jsou k dispozici na http://www.creativecommons.cz respektive https://de.creativecommons.org/.

${ }^{29}$ http://www.sherpa.ac.uk/romeo/index.php
} 


\subsection{Př́nosy a úskalí Open Access}

Přínos otevřeného přístupu pro čtenáře je zcela zřetelný, ale zastavme se u př́nosu pro autora. Statistiky ukazují, že články, zveřejněné v OA režimu jsou viditelnější a jsou proto častěji citovány. Zároveň autor získá rychlou odezvu na svoji práci, protože komunikace s kolegy z celého světa je rychlejší a pružnější. Dochází tak k usnadnění spolupráce a dokonce i širšímu zapojení vědců z dalších oborů, kteří mohou na řešenou problematiku nahližet nově. Výhodu prináší Open Access i ostatním účastníkům procesu vědeckého publikování: vydavatel časopisu získá více čtenářŭ, vyšší citovanost článků přináší vyšší impakt faktor, který zvyšuje hodnotu časopisu. Pracoviště autora se dostává do širšího povědomí vědecké komunity, která má lepší přehled o zpracovávaných tématech, a nedochází k duplikování výzkumu. Volně dostupné odborné publikace mají význam i pro popularizaci vědy, kterou dostávají do širšího podvědomí laické veřejnosti.

Dostat svůj článek do prestižního vědeckého časopisu není ani jednoduché ani rychlé. Se stoupající potřebou vědců publikovat v otevřených časopisech se našli podvodníci - predátoři, kteři ve zlaté cestě Open Access našli zlaté dno. Vědcům nabízejí zveřejnění článku v otevřeném časopise. Jejich jediným cílem je ale pouze vybrat co nejvíce peněz na poplatcích APC. Proto zde neprobíhá klasické recenzní řízení (peer-review), kdy odbornou erudici článku posuzují vědci daného oboru, a až na základě jejich doporučení je přijat do tisku v seriózních časopisech. Predátorské časopisy jsou za peníze ochotné publikovat cokoliv, včetně plagiátů, nezáleží jim na kvalitě příspěvků, a to je pro vědeckou komunikaci i pro důvěryhodnost ostatních $O A$ časopisů velký problém. Proto učíme především mladé vědce a doktorandy, jak tyto tzv. scam journals rozpoznat. Druhým typem predátorů jsou tzv. hijacked journals, kteří si za účelem generování zisku neprávem přivlastňují identitu zavedeného odborného časopisu. Na první pohled je vědec jen těžko rozpozná. Poprvé na problém upozornil Tim Hill v roce 2008, ale v posledních letech se tyto prípady množí. Zatímco v roce 2011 bylo predátorských časopisů přibližně 18, v roce 2016 jich bylo evidováno už 1211.

Většina predátorů používá agresivní marketing. Oslovují hromadnými maily zaměstnance univerzit a vědeckých pracovišt' a nabízi možnost publikování bez jakýchkoliv překážek. Často mají názvy snadno zaměnitelné s některým z prestižních vědeckých časopisů či mají v redakční radě jména renomovaných 
vědců bez jejich souhlasu a vědomí. Někdy uvádí nepravdivý impact factor časopisu, neexistující metriky a identifikátory, falešné údaje. Dokonce se snaží svoji "serióznost" prokázat svým ResearchID a ORCID, což jsou identifikátory určené pro fyzické osoby a zřizuje si je každý vědec sám. Proto je nutné všechny údaje ověrovat. Kromě školení zaměřených na tuto problematiku poskytují vysokoškolské knihovny pomoc při prověřování časopisu, ve kterém chtějí akademici publikovat.

Jako užitečná pomůcka k určení predátorských časopisů byl od roku 2010 hojně využiván seznam Jeffreyho Bealla, který zveřejňoval na svém blogu. Někteří oponenti upozorňovali, že pravidla k zařazení na seznam nejsou zcela jednoznačná, a že se do seznamu mohou dostat některé časopisy neoprávněně. Jako př́klad byly uváděny časopisy nakladatelství Frontiers. V lednu 2017 byl provoz bez bližšího zdůvodnění ukončen a seznam smazán. Přesto zcela nezmizel. Existuje jeho archivní podoba. ${ }^{30}$ Zůstává ale nedoplňován, a to je velký problém, protože predátoři se dokáží velmi rychle přizpůsobit. Změní se název časopisu, nakladatel se objeví pod jiným jménem na jiném místě. Dokonce se z původně seriózního titulu po převzetí novým nakladatelem mǔže stát predátorský.

\subsection{APC a předplatné}

V projektu OpenAPC (Open Data on Article Charges)31 zaznamenávají zúčastněné univerzity a vědecké společnosti údaje o $\mathrm{APC}$, které zaplatily. Průměrná cena článku v plně otevřených časopisech byla 1488 € a v hybridních časopisech $2488 €$. Nejvyšší APC v plně otevřeném časopise zaplatila Max Planck Gesellschaft (7 419 €) a v hybridním časopise University of Oxford (9 070 €). ${ }^{32}$ Vzhledem k podmínce řady projektů publikovat výsledy v OA režimu, počet otevřených článků v hybridních časopisech stoupá. Předplatitelé proto právem požadují, aby byla výše předplatného úměrně tomuto nárůstu snížena. Vydavatelské domy se tomu zatím úspěšně brání. Ukazuje se ovšem, že větší naděje na úspěch ve vyjednávání mají velká konsorcia. Velmi aktivní a úspěšní jsou v tom Holand’ané33. Holandské asociaci univerzit

\footnotetext{
30 https://web.archive.org/web/20170112125427/https://scholarlyoa.com/publishers/

31 https://intact-project.org/openapc/

32 https://github.com/OpenAPC/openapc-de

33 http://www.magazine-on-the-spot.nl/openaccess/eng/index.html
} 
se ve spolupráci s knihovnami a za silné podpory vlády podařilo vyjednat u nakladatelů řadu ústupkư ${ }^{34}$.

V Německu vznikl projekt DEAL ${ }^{35}$, jehož cílem je uzavřít výhodnější celostátní licenční smlouvy s významnými nakladateli vědecké literatury a změnit status quo v procesu vyjednávání právě s ohledem na zaplacené APC. Projekt byl iniciován Aliancí německých vědeckých organizací (Allianz der deutschen Wissenschaftsorganisationen) reprezentovanou Konferencí rektorů HRK.

Protože jsou APC považovány v řadě projektů za uznatelné náklady, zajímají se také nadace financující vědu o jejich výši. Například nadace Wellcome Trust $^{36}$ a nadace Gatesových ${ }^{37}$ se prostřednictvím F100resarch vydávají cestou vlastních publikačních portálů s otevřeným recenzním řízením (open peer review). Práce související s jejich projekty jsou zveřejněny okamžitě v OA režimu a k nim jsou časem přidány recenzní posudky, takže čtenář může vidět články čekající na recenze, články doporučené recenzenty, doporučené s výhradami i články nedoporučené. Každý vidí jména recenzentů a může si přečíst jejich posudky, reakce autorů i komentáře přihlášených uživatelů.

\subsection{Institucionální a politická podpora Open Access}

Grantové agentury a vládní instituce, které financují vědu, se postupně přiklání k názoru, že pokud je věda financována z veřejných peněz, měly by její výsledky být odborné veřejnosti volně dostupné. Významným krokem byla iniciativa Evropské komise, která pro některé oblasti 7. rámcového programu stanovila povinnost řešitelů, zajistit otevřený přístup k publikačním výsledkům. V programu Horizont 2020 je tato povinnosti ještě rozširrena: týká se všech recenzovaných vědeckých článků, které souvisí s výsledky grantu, a obsahuje silné doporučení u ostatních typů vědeckých publikací. V tzv. pilotních projektech (OA data pilot) je povinnost zveřejnit v Open Access režimu i všechna výzkumná data, včetně příslušných metadat, a vytvořit tzv. Data Management Plan. Data mohou být zveřejněna bud' spolu s příslušnou publikací v OA datovém časopise (např. Open Health Data, Scientific Data) či v datovém repozitári (např. Zenodo, Fishare, DataDryad).

\footnotetext{
34 http://openaccess.nl/en/in-the-netherlands/publisher-deals\#row

35 https://www.projekt-deal.de/about-deal/

${ }^{36}$ https://wellcomeopenresearch.org/

${ }^{37}$ https://gatesopenresearch.org/
} 
Prostřednictvím projektu OpenAire ${ }^{38}$ byla zajištěna potřebná infrastruktura pro ukládání dat a zázemí pro zúčastněné repozitáre. Orientaci v požadavcích nadací a grantových agentur, které vyžadují otevřené zpřístupnění výsledků výzkumu, usnadňuje služba SHERPA/Juliet. ${ }^{39}$ Seznam časopisů, které jsou v souladu s požadavky poskytovatele financí pro Open Access je možné ověřit na SHERPA/Facts. ${ }^{40}$

Otev̌̌ený př́stup k vědeckým informacím se stal nedílnou součástí zásadních strategických dokumentů Evropské Unie. Např. Doporučení komise ze dne 17. července 2012 o př́stupu k vědeckým informacím a jejich uchovávání (2012/417/EU) uvádí:

(5) Cílem politik otevřeného přístupu je poskytnout čtenárüm bezplatně přistup k recenzovaným vědeckým publikacím a údajům z výzkumu v co nejranější fázi jejich širrení a umožnit používání a opakované využívání výsledků vědeckého výzkumu. Tyto politiky by se měly provádět s ohledem na problematiku práv duševního vlastnictví

(6) Politiky otevřeného prǐstupu k výsledkưm vědeckého výzkumu by se měly vztahovat na veškerý výzkum financovaný z veřejných prostředkư. ${ }^{41}$

Roku 2016 vyhlásila Rada Evropské unie pro konkurenceschopnost, že do roku 2020 by měly být volně dostupné všechny vědecké práce financované $z$ veřejných peněz. Ačkoliv se $k$ tomuto cíli a podpoře Open Access mnozí hlásí, přesto jeho naplňování vázne. Podle Roberta-Jana Smitse, který byl jmenován zvláštním vyslancem Evropské unie pro Open Access, je to způsobeno současným hodnocením vědců podle počtu publikací v prestižních časopisech $s$ vysokým impakt faktorem. Proto hodlá předložit doporučení, které nebude zaměřeno pouze na otev̌rený prístup $\mathrm{k}$ vědeckým publikacím, ale na celý systém včetně změn, které je potřeba udělat, abychom v termínu dosáhli vytčeného cíle. ${ }^{42}$

\footnotetext{
38 https://www.openaire.eu/

39 http://v2.sherpa.ac.uk/juliet/

$40 \mathrm{http}: / /$ www.sherpa.ac.uk/fact/index.php?la=en

41 http://eur-lex.europa.eu/legal-content/CS/TXT/?uri=CELEX:32012H0417

42 https://horizon-magazine.eu/article/open-access-scientific-publications-must-becomereality-2020-robert-jan-smits_en.html
} 
Společnost Maxe Plancka, Helmholtz a Německá knihovnická asociace patřily k prvním signatářưm Berlínské deklarace v roce 2003 a stojí v čele hnutí Open Access dosud. Napřílad sdružení Helmholtz deklarovalo, že bude $60 \%$ jejich publikací OA do roku 2020 a do roku 2050 všechny. Již v roce 2014 zakotvila Spolková vláda do autorského zákona podmínky usnadňující ukládání vědeckých publikací do repozitár̆u. Od roku 2016 má Německo schválenou oficiální národní Open Access strategii (Open-Access-Strategie für Deutschland) vydanou Federálním ministerstvem pro vzdělávání a vědu (Bundesministerium für Forschung und Bildung). ${ }^{43}$

Přístup k Open Access v České republice byl donedávna zcela závislý na jednotlivých institucích. Hybnou silou Open Access byly a jsou především univerzitní knihovny, které myšlenku nejen propagují, ale většinou i zřizují a spravují institucionální repozitáře. Na většině univerzit je proto zásluhou knihoven vytvořena potřebná infrastruktura pro ukládání publikací v OA režimu. Počty opravdu otevřených vědeckých publikací jsou ale v jednotlivých institucích velmi rozdílné. Mnohé repozitáře svým návštěvníkům zpřistupñují pouze bakalářské a diplomové práce. Teprve 14.06.2017 schválila česká vláda Národní strategii otevřeného př́stupu k vědeckým informacím na léta 2017-2020.

\subsection{Situace u projektových partnerů}

Mezinárodní hnutí Open Access je podporováno především akademickými knihovnami, proto byla jeho podpora logicky zahrnuta i do projektu Prostor učení - Knihovnická informační platforma. Našim cílem bylo propagovat myšlenku otevřeného přístupu mezi uživateli. Zjistit, jaký je stav především u zahraničních projektových partnerů, vyměnit si zkušenosti s propagací Open Access mezi svými uživateli, s komunikací a podporou managementu univerzity, a v neposlední řadě se správou repozitářu.

Vedení Technické univerzity Chemnitz podporuje otevřené publikování svých vědeckých pracovníků již od roku 2006, kdy byla zformulována Open Access politika instituce. Roku 2015 univerzita podepsala Berlínskou deklaraci a téhož roku svoji politiku otevřeného přístupu ještě rozšririla. Podporována je jak zlatá, tak zelená cesta Open Access.

${ }^{43}$ https://www.bmbf.de/pub/Open_Access_in_Deutschland.pdf 
Motorem realizace otevřené vědy na Technické univerzitě Chemnitz je Univerzitní knihovna, která má Open Access politiku ještě propracovanějšíi4 ${ }^{4}$. Již několik let nabízí služby a know-how pro otevřené publikování. Poskytuje platformu pro správu a publikování vlastních OA časopisů. Její Open Access tým pořádá semináře, školení a poskytuje individuální konzultace. Samožrejmě se věnuje i propagaci otevřeného přístupu na webových stránkách knihovny, ${ }^{45}$ blogu ${ }^{46}$ i v univerzitním měsíčníku TUCinside. ${ }^{47}$

Knihovna má ve své správě fond na podporu zlaté cesty $0 A$, který je tvořen částečně z rozpočtu knihovny a dále financemi od Německé výzkumné společnosti (DFG - Deutsche Forschungsgemeinschaft). Knihovna má podrobně propracované podmínky k využití fondu. Poskytuje příspěvek pouze do plně otevřených časopisů obsažených v DOAJ s recenzním řízením (peer review). APC může být maximálně 2000 € a zároveň článek není součástí projektu, ve kterém Ize na poplatek získat príspěvek. Články musí být uloženy i do repozitáre Monarch Qucosa. První článek fakulty je plně hrazen z fondu, u každého dalšího je vyžadována 10\% finanční spoluúčast pracoviště autora. Podrobné podmínky jsou uvedeny na stránkách knihovny. ${ }^{48}$

Univerzitní knihovna má na starosti repozitár Monarch Qucosa ${ }^{49}$, do kterého jsou ukládány především vědecké práce: konferenční př́spěvky (conferens paper), články (articel), výzkumné zprávy, dizertační práce, sborníky a publikace vydávané Univerzitním nakladatelstvím. V současnosti je zde uloženo 3604 dokumentů z nich je 90\% Open Access.

Také na Vysoké škole Zittau/Görlitz roste význam otevřeného publikování. Někteří vědečtí pracovníci již delší dobu v Open Access publikují a sami ho propagují, pro další se problematika stává aktuální především v souvislosti s požadavky poskytovatelů dotací. Akademici mohou ukládat své publikace do saského repozitáře Qucosa ${ }^{50}$ prostřednictvím SLUB.

Vysokoškolská knihovna v rámci projektu začala své uživatele informovat o problematice otevřeného publikování formou seminářů a informačních mailů.

\footnotetext{
${ }_{44}$ https://www.tu-chemnitz.de/ub/publizieren/openaccess/\#policy

45 https://www.tu-chemnitz.de/ub/publizieren/openaccess/index.html

${ }^{46}$ https://blog.hrz.tu-chemnitz.de/bibo/

${ }^{47}$ https://www.tu-chemnitz.de/rektorat/rektor/newsletter.php

48 https://www.tu-chemnitz.de/ub/publizieren/openaccess/\#finanz

49 http://monarch.qucosa.de/

50 http://www.qucosa.de/
} 
Byly vytvořeny webové stránky se základními informacemi o Open Access. ${ }^{51}$ Snaha vysokoškolské knihovny byla korunována úspěchem: v současnosti je rozpracována OA strategie Vysoké školy Zittau/Görlitz, která by měla projít schvalováním vedení.

Západočeská univerzita v Plzni podepsala Berlínskou deklaraci v roce 2015, ale nemá doposud zformulovanou a schválenou Open Access politiku. Univerzitní knihovna se do propagace OA zapojila v roce 2010. Pravidelně porádá semináře zaměřené na problematiku otevřeného publikování, má webové stránky věnované Open Access, ${ }^{52}$ vydává letáčky a poskytuje individuální konzultace, nejčastěji v otázkách kvality OA časopisů. Každoročně se zapojuje do mezinárodní propagační akce Open Access week.

V roce 2012 knihovna zprovoznila institucionální repozitář Digitální knihovnu ${ }^{53}$ pod systémem DSpace. Repozitář obsahuje 27483 dokumentů ${ }^{54}$ a je rozčleněn na tři základní kolekce: publikační činnost (publications) - obsahuje články, konferenční sborníky a další práce autorů ZČU (5 648 dokumentů) a dále články a statě z časopisů a sborníků vydávaných na univerzitě (7 052 dokumentů); vysokoškolské kvalifikační práce (thesis) - bakalářské a diplomové práce (14 496 dokumentů) a otevrené vzdělávací zdroje (open resources) - dokumenty, média, kurzy, modely či software využívané k výuce, učení, hodnocení a výzkumné účely (93 dokumentů). Až na výjimky je vše v Open Access režimu. Digitální knihovna ZČU má české a anglické jazykové rozhraní. U každého příspěvku i kolekce je možné si prohlédnout statistiky využívání.

Za dobu trvání projektu bylo přidáno téměř čtyři tisíce dokumentů. Byla upgradována verze DSpace 5 a vytvořena nová grafická podoba Digitální knihovny. Základní soubor funkcí byl rozšir̃en o doplňkové služby a nástroje. Knihovna byla propojena s dalšími univerzitními systémy: studijní agendou STAG, discovery sytémem Summon a se softwarem k evidenci publikací OBD. Data uložená v Digitální knihovně jsou importována do národního repozitáře šedé literatury NUŠL, ${ }^{55}$ do agregátoru repozitářu OpenAire a do registru repozitářu OpenDOAR.

${ }^{51}$ https://hsb.hszg.de/ueber-uns/projekte/lernraum-2016-2018/open-access.html

52 http://knihovna.zcu.cz/open-access/OpenAccess.html

53 https://dspace.zcu.cz/

54 Stav z května 2018.

55 http://www.nusl.cz/ 
V pilotním provozu běží odevzdávání všech článků a konferenčních príspěvků ze dvou pracovišt ZČU. Ty jsou importovány do Digitální knihovny a jejich plné texty jsou zveřejňování dle politiky nakladatelù zveřejňované $v$ databázi SHERPA/ROMEO. Pro ostatní autory je ukládání publikací do repozitáře zatím dobrovolné. Kromě toho využívá řada autorů ZČU také zlatou cestu Open Access. APC jsou hrazeny částečně z projektů a částečně z pracoviště autora. Univerzita nemá žrízen žádný podpưrný fond.

Univerzitní knihovna Technické univerzity v Liberci podepsala Berlínskou deklaraci prostřednictvím Asociace knihoven vysokých škol ČR v roce 2012. Oficiální Open Access politiku zatím univerzita nemá. 0 problematice otevřeného publikování informuje knihovna na přednáškách pro veřejnost, v jednorázových kurzech, téma zařazuje i do výuky a poskytuje také individuální konzultace. Open Access propaguje letáky, plakáty, na webových stránkách i sociálních sítích. V rámci Open Access Week se knihovna převlékne do oranžové.

Univerzitní knihovna provozuje svưj repozitáŕ56. pod DSpace Uloženo je zde 22177 dokumentů, z nich je otevřených téměř 70\%. Největší část tvoří bakalářské, diplomové, disertační a habilitační práce (20 028 dokumentů). Dále jsou zde archivovány publikace autorů Technické Univerzity v Liberci (421 dokumentů), Open Access časopisy vydávané na univerzitě (596 dokumentů) a výukové materiály. Vedení univerzity v květnu 2018 rozhodlo 0 zveřejnění všech vysokoškolských prací a to i retrospektivně. Až na objektivně zdůvodněné výjimky budou všechny práce v režimu Open Access.

Pravidelně jsou importována metadata publikací univerzitních autorů z citačních databází WoS a Scopus. K nim knihovna dohledává a pripojuje plné texty.

$V$ průběhu projektu přešla knihovna na verzi 6.2 DSpace, přemapovala repozitáŕ, vyčistila a obohatila metadata do strojově čitelnější podoby. Zdigitalizovala a uložila v repozitáŕi univerzitní časopis ACC Journal od roku 2009 do současnosti. Prưběžně digitalizuje všechny výzkumné a technické zprávy Technické univerzity v Liberci.

Repozitár byl propojen se studijní agendou STAG, což umožňuje prímý import plných textů závěrečných prací. Dále pracovníci knihovny participují na zavedení datových kanálů z univerzitního systému na evidenci výsledků vědy

$\overline{{ }^{56} \text { https://dspace.tul.cz }}$ 
a výzkumu publikace.tul.cz. To umožní po uplynutí embarga zveřejnit výsledky excelentního výzkumu Technické univerzity v Liberci.

\subsection{Systémy repozitářů projektových partnerů}

Němečtí partneři využívají repozitář Qucosa, který nad systémem Fedora vyvinula a spravuje SLUB (Sächsische Landesbibliothek -Staats- und Universitätsbibliothek Dresden). Jeho jednotlivé části mohou sloužit jako repozitáře jednotlivých institucí. Tímto způsobem ho využívá 8 organizací v Sasku včetně Technické univerzity Chemnitz. Zároveň mohou prostřednictvím SLUB ukládat své práce i autoři z dalších univerzit a vysokých škol. Takto je využíván na Vysoké škole Zittau/Görlitz. Vyhledávat je možné mezi všemi uloženými dokumenty nebo jen v konkrétním repozitárii.

Publikační server Qucosa slouží k dlouhodobé archivaci dokumentů a jejich zpř́stupňování. Všechny práce mají trvalý identifikátor (urn: nbn) a jsou popsány pomocí strukturovaných metadat. Dokumenty jsou vyhledatelné i v katalozích zúčastněných knihoven a Německé národní knihovny i ve službě Google Scholar.

Oba čeští partneři používají open source systém DSpace, který používá 43\% světových repozitářu. Je určen akademickým, neziskovým i komerčním organizacím, které vytvářejí otevřené digitální úložiště. Mezi jeho přednosti patří jednoduchá instalace, úplná přizpůsobitelnost i návaznost na ostatní systémy. Samožrejmostí jsou různé jazykové mutace systému.

DSpace uchovává a umožňuje snadný a otevřený přistup ke všem typům digitálního obsahu, včetně textu, obrázků, pohyblivých obrazů, videí, mpegů a datových sad v různých formátech. Jednotlivým dokumentům je přiřazen trvalý identifikátor handle, díky tomu je indexován Googlem.

Uložené a archivované dokumenty lze prohližet (browse) nebo vyhledávat (search) podle kolekcí, autorů, názvů, klíčových slov či druhu dokumentů. Vyhledávat je možné i s použitím boolovských operátorů (AND, OR, NOT). Systém uživatele informuje o nově přidaných položkách, umožňuje nastavit různá přístupová práva $\mathrm{k}$ dokumentům nebo pro jednotlivé skupiny uživatelů.

Všechny repozitáře projektových partnerů jsou př́stupné z webových stránek projektu lead partnera - Technické univerzity Chemnitz. ${ }^{57}$

57 https://www.tu-chemnitz.de/ub/projekte-und-sammlungen/projekte//rbip/infoplattform/ 


\subsection{Společné akce v rámci projektu}

V záři 2017 proběhl na Technické univerzitě v Liberci společný workshop, na kterém se projektoví partneři informovali o aktuálním stavu Open Access na svých institucích. Kolegové z Univerzitní knihovny Technické univerzity Chemnitz seznámili projektové partnery nejprve s celkovou situací v Německu a pak na univerzitě. Inspirativní je způsob využívání publikačního fondu se spoluúčastí autora. Technická univerzita v Liberci a Západočeská univerzita v Plzni na workshopu také představily své institucionální repozitáře pracující pod systémem DSpace. Na všech třech univerzitách je kromě možnosti uložit práci do repozitáře, využívána i zlatá cesta otevřeného publikování.

Pro knihovnu Vysoké školy Zittau/Görlitz bylo téma poměrně nové. Na škole sice někteří vědci ke své práci otevřené publikace využívají, méně z nich však v OA režimu publikuje. Vzhledem k tomu, že iniciativa Open Access je aktuální především pro vysokoškolské knihovny, nebyla do ní zatím zapojena ani Krajská knihovna v Liberci.

Říjen je již tradičně dobou, kdy probíhá mezinárodní akce Open Access Week, při níž zejména akademické knihovny propagují myšlenku otevřeného publikování. Každoročně je vyhlášeno společné heslo, pod kterým jsou akce pořádány. Heslo pro rok 2017 Open Access. Open in order to... se stalo i názvem plzeňského říjnového semináře informačního vzdělávání, určeného především mladým vědcưm.

Program zahrnoval zevrubný náhled do problematiky vědeckého publikování v režimu Open Access, od základních informací až po specializované přednášky, zaměřené na predátorské časopisy nebo na analýzu nákladů českých univerzit na publikační poplatky (APC). Účastníci semináře byli seznámeni s největšími českými repozitáři i s nejdůležitějšími celosvětovými registry se zaměřením na Open Access. Samostatně byl prezentován repozitář hostitelské univerzity (Digitální knihovna ZČU) včetně jeho propojení na software, sloužící ke sběru informací o publikacích univerzitních autorů (OBD). Seminář navštívilo 43 účastníků, především doktorandů, ze všech pěti organizací, zapojených do projektu.

V říjnu 2018 se v rámci Open Access Week s tématem Designing Equitable Foundations for Open Knowledge konala třídenní výměnná stáž na Západočeské univerzitě v Plzni. Z každé ze spolupracujících knihoven přijeli dva pracovníci 
se zájmem o problematiku otevřeného publikování. Vzhledem k rozdílné mî̃e zkušeností jednotlivých účastníků s $0 A$, byla zařazena přednáška se stručným úvodem do problematiky a praktická ukázka postupu při ověřování predátorského časopisu. Projektoví partneři se detailně seznámili s plzeňským repozitářem a jeho napojením na další univerzitní informační systémy i s konceptem otevřeného vzdělávání. Při exkurzích po odborných pracovištích Univerzitní knihovny mohli vidět, jak je zde Open Access propagován.

\subsection{Výsledky a závěrečné hodnocení milníku}

Téma Open Access do projektu Prostor učení zcela logicky patří, protože rozšiřuje oblast přistupu k znalostem. Milník směřoval k prohloubení povědomí o Open Access mezi uživateli, ale zejména k výměně zkušeností se zahraničními partnery. Příklady dobré praxe v Německu a v České republice a především na jednotlivých univerzitách, posílily metodické a vzdělávací kompetence knihovníkủ a obohatily o nové poznatky i uživatele knihoven. Zvlášt zajímavé bylo srovnání vládní podpory OA v Německu a v České republice. Př́nosem byly i neformální debaty o problematice, které probíhaly při každém setkání, s cílem posílit efektivitu přednášek, přispět k implementaci nastolených témat a podpořit sebereflexi účastníků ve vztahu ke konkrétním požadavkům uživatelů, univerzit a grantových agentur (např. vytvoření www stránek o 0A, zř́zení fondu na podporu OA publikování, príprava směrnice univerzity). Reagovaly též na momentální potřeby účastníků (i vzhledem k různé úrovni podpory OA na jednotlivých univerzitách) a na otázky a problémy, které nebyly zařazeny do oficiálního programu (např. datové repozitáře, spolupráce s projektovými centry). Vzájemná inspirace a čerpání ze zkušeností ostatních, pomohly účastníkům projektu uvědomit si své slabé a silné stránky, dodaly podněty ke zlepšení. Vzhledem ke vzrůstajícímu vlivu OA a jeho potřebě v praktickém fungování vědeckého publikování byl tento milník velmi prínosný pro všechny účastníky. Celkově milník hodnotíme po všech stránkách jako smysluplný. 


\title{
INFORMAČNÍ VZDĚLÁVÁNÍ V PR̆ESHRANIČNÍM PROSTORU UČENÍ
}

aneb Co musí modul informačního vzdělávání zprostředkovávat

\author{
Angela Malz \\ Václav Ovčačík
}




\subsection{Projektové aktivity}

Skutečnost, že knihovny nabízejí pro uživatele různá školení a prohlídky není nic nového. Uvést své uživatele do pravidel užívání knihovny a možností rešerše totiž patří k základním úkolům knihoven. Dříve se museli návštěvníci knihoven seznámit s lístkovými katalogy, tištěnými bibliografiemi nebo různými príručkami. Dnes, v době digitálních knihoven, musí uživatelé umět zacházet s databázemi, odbornými portály a online katalogy. Mimoto se musí naučit vyfiltrovat z množství informací ty, které potřebují pro řešení daného problému tzn. musí tyto informace zpracovat a vyhodnotit. K tomu je zapotřebí schopnost, kterou označujeme jako informační kompetenci.

Při prípravě žádosti k projektu Prostor učení - Knihovnická informační platforma se brzy ukázalo, že do projektu by mělo být jako samostatný milník zahrnuto i téma informačního vzdělávání.

V projektové žádosti k tomu stojí:

„Pod pojmem informační kompetence se rozumí schopnost najít, zpracovat a správně vyhodnotit informace. Knihovny usilují o lepší zapojení informační kompetence do výuky, výzkumu a studia. K tomu má sloužit speciálně vyvinutý, didaktický modul informační kompetence v tištěné a elektronické podobě. $V$ rámci projektu budou zváženy další možné koncepty."

K realizaci milníku byly naplánovány následující aktivity:

- 09.05.2017 Den informačního vzdělávání v Univerzitní knihovně Chemnitz

- 25.04.2017 Den informačního vzdělávání v knihovně Zittau (webináŕ)

- 26.09.2018 Workshop v Univerzitní knihovně Chemnitz

Tyto aktivity směřovaly dvěma směry. Na jedné straně se měli uživatelé knihoven v rámci akcí k informačnímu vzdělání seznámit s možnostmi rešerše a především médii zakoupenými v rámci projektu. Tento cíl sledovaly oba dny informačního vzdělávání v Chemnitz a Zittau. Akcí se zúčastnili mladí vědci a studenti partnerských institucí. V Univerzitní knihovně Chemnitz jim byl zprostředkován náhled do digitální knihovny a dozvěděli se zajímavosti o clusteru MERGE. Den informačního vzdělávání plánovaný v Zittau byl pojat jako webinár. Zúčastněné knihovny představily predevším ta média, která byla pořízena v rámci projektu. Zároveň byla zapojena technika rovněž 
nakoupená během milníku 2 (Technické vybavení). Na druhé straně se knihovníci zabývali problematikou informačního vzdělávání. Nejprve toto téma teoreticky zpracovali, srovnali různé koncepty a poté vytvořili modul informačního vzdělávání.

Ve dnech 23. a 24. května 2017 se setkali knihovníci ze všech projektových knihoven $v$ rámci praktické stáže $v$ Liberci a $v$ Görlitz. Vedle témat $\mathrm{k}$ technickému vybavení Prostoru učení si účastníci vyměnili zkušenosti v oblasti zprostředkovávání informačního vzdělávání. Velmi brzy bylo zřejmé, že knihovny přicházejí do projektu s rozdílnými předpoklady. Nejvíce zkušeností se zprostředkováváním informačního vzdělávání měly univerzity v Chemnitz a Plzni. Během živých diskuzí byl vytyčen rámec modulu informačního vzdělávání: Jaké cíle mají být dosaženy? Jaké obsahy budou zprostředkovány? Na jaké cílové skupiny se bude modul orientovat? Pomocí jakých opatření budou tyto cíle dosaženy? Jako výsledek vznikla grafika, která sloužila jako podklad pro další práci.

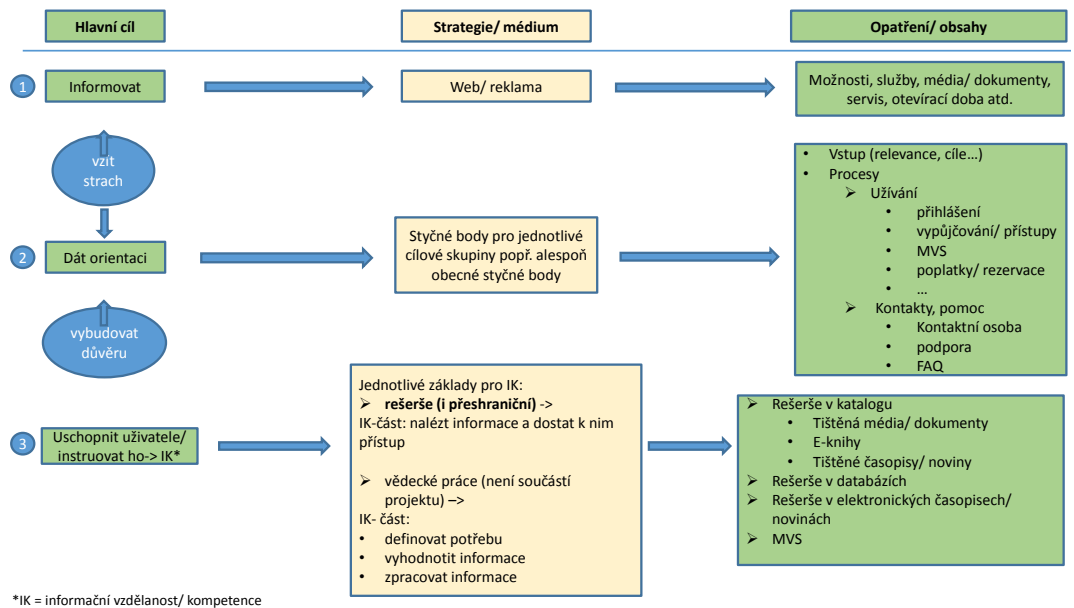

Obrázek 1. Základ pro modul informačního vzdělávání. Autor: Falk Maiwald.

\subsection{Vznik modulu informačního vzdělávání}

3. srpna 2017 se setkali spolupracovníci Vysokoškolské knihovny Zittau, Univerzitní knihovny v Liberci a Krajské vědecké knihovny v Liberci, aby dále pracovali na milníku informační vzdělávání. Společně sestavili plán, uveřejnit v systému Moodle pro každou knihovnu nejdůležitější informace k využívání 
knihovny ve formě často kladených otázek (FAO) a vypracovali k tomu odpovídající katalog otázek.

26. záři 2017 pak na workshopu v Univerzitní knihovně v Liberci prezentovali aktuální stav práce. V závěru však nebylo možné jednoznačně rozhodnout, zda je systém Moodle skutečně tou nevhodnější platformou pro modul informačního vzdělávání. Výhrady měli především němečtí projektoví partneři, kteří Moodle jako vzdělávací platformu nevyužívají.

Diskuze proto pokračovala i na workshopu 14. února 2018 v Univerzitní knihovně v Plzni. Projektoví partneři se shodli na tom, že na Moodle bude pohlíženo jako na další koncept (viz kapitola 3.4).

Lead partner byl pověřen navrhnout nový koncept pro „speciálně vyvinutý, didaktický modul informačního vzdělávání v tištěné a elektronické formě“.

Univerzitní knihovna Chemnitz má v oblasti informačního vzdělávání bohaté zkušenosti. Se zavedením bakalářských a magisterských studijních oborů nabízí Univerzitní knihovna Chemnitz od zimního semestru 2006/2007 různé akce k informačnímu vzdělávání, které jsou částečně zaintegrovány do studijních programů. Uživatelé knihovny však vyjádřili přání, získávat informace nezávisle na tom, kde a kdy se nacházejí. Univerzitní knihovna Chemnitz proto vyvinula vlastní e-learningové kurzy, pro což využila zkušenosti ostatních knihoven např. Univerzitní knihovny Konstanz nebo zkušenosti z projektu lotse ${ }^{58}$.

Na základě řady zkušeností Univerzitní knihovny Chemnitz vznikl modul informačního vzdělávání, který je použitelný i pro další knihovny a je pro všechny projektové partnery k dispozici jak v německém tak v českém jazyce.

Modul informačního vzdělávání je koncipován tak, aby byl využitelný pro různé cílové skupiny a různé problémy. Podle potřeby je možné jednotlivé moduly různými způsoby skládat nebo využivat nezávisle na sobě.

Modul může být využit také všemi dalšími zainteresovanými knihovnami. Individuální obsahy - vztažené ke konkrétní knihovně - je třeba přizpůsobit. Nezávislé informace je možné využivat beze změn. Modul59 je zveřejněn pod licencí CC BY.

58 https://www.ulb.uni-muenster.de/ulb-tutor/lotse/

${ }^{59}$ https://www.tu-chemnitz.de/ub/kurse-und-e-learning/elearning/studierende/ikonline.html 
V zárí 2018 se konal poslední workshop milníku informačního vzdělávání v Univerzitní knihovně Chemnitz. Zde byla doladěna finální verze modulu informačního vzdělávání jak v německé tak v české verzi.

\subsection{Struktura modulu informačního vzdělávání}

\section{Modul 1 - Univerzitní knihovna a katalog}

V tomto modulu zodpovíme následující otázky:

- Jak vlastně fungují vědecké knihovny?

- Co všechno najdu v katalogu a co ne?

- Jak najdu knihu v knihovně? A jak si ji pak vypůjčím?

- Semestrální aparáty - co je to a má je každá knihovna?

- Kde můžu hledat dál, když moje knihovna nemá hledanou knihu nebo časopis?

Obsah:

- Univerzitní knihovny

- Katalog

- Vyhledávání

- Tipy

- Totoriál katalog (DE)

- Výsledky

- Ranking

- Omezení

- Zobrazení exempláre

- Totoriál zobrazení exempláře (DE)

- Rozšrĩ̃ené vyhledávání

- RVK

- Príklady

- Semestrální aparáty

- Nadregionální vyhledávání

- KVK

- Závěrečný test (DE) 


\section{Modul 2 - Příprava rešerše}

V tomto modulu zodpovíme následující otázky:

- Jak strukturovat rešerši, abyste ušetřili čas?

- Co jsou databáze a jak poznáte, které jsou pro Vás vhodné?

Obsah:

- Databáze - pojem

- Databankový informační systém

- Tutoriál Databankový informační systém (DE)

- Model pěti kroků:

- Co hledám?

- Tutoriál Tématická analýza (DE)

- Shromáždění hledaných pojmů

- Co potřebuju?

- Kde hledám?

- Jak hledám?

- Prohlédnutí a modifikace

- Závěrečný test (DE)

\section{Modul 3 - Techniky vyhledávání a funkce v databázích}

V tomto modulu zodpovíme následující otázky:

- Jaké techniky vyhledávání můžete používat ve vyhledávání v databázích?

- Jaké další komfortní funkce nabízí databáze?

Obsah:

- Techniky vyhledávání

- Vyhledávací pole/ vyhledávané pojmy

- Volba vyhledávacího pole

- Nalezení vyhledávaného pojmu

- Předmětové heslo/ klícové slovo

- Tutoriál klíčová slova (DE)

- Tezaurus

- Index 
- Nástroje pro vyhledávání

- Truncation

- Vyhledávání frází

- Booleovské operátory

- Tutoriál Booleovské operátory (DE)

- Funkce v databázích

- Search History

- Alerting

- Pomocné funkce

- Závěrečný test (DE)

\section{Modul 4 - Rešerše na internetu}

V tomto modulu zodpovíme následující otázky:

- Jaké vyhledávače a webové stránky jsou vhodné pro vědecké práce?

- Jak můžete na internetu efektivně hledat informace, především vědecké texty?

- Jaké zdroje z internetu můžete použít pro svou vědeckou práci?

Obsah:

- Informační zdroje na internetu

- Obecné vyhledávače

- Surface, Deep \& Darknet

- Speciální vyhledávače

- Př́klady speciálních vyhledávačů

- Odborné stránky

- Důležité odborné stránky

- Odborné stránky UK Chemnitz

- Techniky vyhledávání

- Príklady

- Prohlédnutí a modifikace

- Závěrečný test (DE) 


\section{Modul 5 -Získání literatury}

V tomto modulu zodpovíme následující otázku:

- Jak získáte literaturu, kterou jste nalezli při rešerši?

Obsah:

- Vypůjčení a objednávka

- Vyhledání plných textů

- Tutoriál Dostupný dokument (DE)

- Meziknihovní výpůjční služba / Služby pro doručení dokumentů

- Meziknihovní výpůjční služba

- Tutoriál Meziknihovní výpůjční služba - objednávka (DE)

- Služby pro doručení dokumentů

- Návrh na pořízení

- Závěrečný test (DE)

\section{Modul 6-Citace a plagiáty}

V tomto modulu zodpovíme následující otázky:

- Co všechno obsahuje citace?

- Jak byste měli citovat a co můžete převzít z vědeckých prací?

- Co je plagiát?

Obsah:

- Citace

- Pravidla citací

- Prímé/ neprímé citace

- DIN

- Plagiáty

- Definice

- Př́klady

- Následky plagiátorství

- Autorská práva

- Závěrečný test (DE) 


\subsection{Koncept v Moodlu}

Modul k informačnímu vzdělávání ${ }^{0} v$ prostředí Moodle vznikal postupně po celou dobu trvání projektu Prostor učení - Knihovnická informační platforma. Na jednom z prvních setkání k tomuto tématu byl zvolen otevřený software Moodle, jelikož je to v České Republice nejpoužívanější e-learningový systém (v rámci diskuzí byl zvažován i v německu užívaný systém OPAL = Online-Plattform für Akademisches Lehren und Lernen). I presto, že od něj němečtí partneři v průběhu projektu upustili, protože se u nich tato platforma netěší takové oblibě, prisspěli kolegové z Chemnitz a Zittau/Görlitz v procesu návrhu i tvorby tohoto modulu svými návrhy, zkušenostmi a připomínkami.

Z prvotního setkání vyplynulo několik důležitých faktorů, se kterými se dále počítalo v celé koncepční fázi modulu v Moodlu.

1. Architektura modulu musí být dostatečně otevřená, aby umožnila partnerům upravovat jednotlivé části, protože partnerské knihovny mají rozdílné poť̌eby a cíle $\rightarrow$ víceúrovñová architektura

2. Největši prínos bude mít zaměření na problémy, se kterými se uživatelé běžně potýkají $\rightarrow$ forma často kladených otázek a odpovědí

Základní seznam otázek vytvořila Univerzitní knihovna Technické univerzity v Liberci a partneři poté dostali možnost otázky komentovat a upravovat dle svého uvážení. Vzniklo tak mnoho unikátních otázek zaměřených na konkrétní služby partnerských knihoven.

Primární rozdělení bylo na kategorie otázek, zde partneři zvolili dělení podle cílových skupin - Studenti a Akademici - a přidali kategorii Základní služby s obecnými dotazy na fungování knihovny.

Po několika diskuzích byla přidána ještě úroveň samotných knihoven. Původní koncept totiž počítal s větším propojením jednotlivých knihoven, kdy by na každou otázku existovala obecná odpověd" - napríklad popis meziknihovní výpưjční služby - a až poté by si uživatel vybral specifickou knihovnu a dostal se ke konkrétním pravidlům instituce. Nakonec ale z důvodu velkých rozdílů v poskytovaných službách a pro zjednodušení orientace uživatelům skončilo rozdělení podle knihoven na první pozici. Tím jsme se vyhnuli prípadům, kdy by vznikali obecné odpovědi pro službu, kterou stejně poskytuje pouze jeden z partnerů - napríklad priidělování ORCID - nebo př́padům, kdy by uživatele

${ }^{60}$ https://elearning.tul.cz/course/view.php?id=3335 
obecná odpověd' pouze oddalovala od požadované informace. Některé služby se navíc liší jak v jednotlivých státech, tak i v rámci nich (fungování Krajské vědecké knihovny a knihoven univerzitních).

Současný systém tedy vypadá takto:

- Úvodní strana s představením projektu a výběrem partnerské knihovny

- Úvodní strana vybrané knihovny s odkazy na web, katalog atd. (repozitáře, sociální sítě) a výběrem kategorie otázek

- Otázky

- Odpovědi

Co se týče přidávání obsahu, každý partner dostal př́stup k platformě a byl seznámen se základními pravidly a možnostmi systému jak na společných setkáních, tak přímo v Moodlu. Dále bylo založeno diskuzní fórum Návrhy a připomínky/Anregungen und Kommentare a Oznámení. Nakonec se ale většina komunikace řešila přes e-maily, prípadně telefonicky, takže kromě návodu na práci v kurzu byla tato fóra nevyužita. Partneři nezávisle přidávali obsah do vytvořených sekcí v jedné jazykové verzi s tím, že byl domluven postup při následném překládání. Do Moodlu Technické univerzity v Liberci byl pro tento účel přidán Multi-language content filter, který umožňuje jednoduše vkládat tagy pro jednotlivé jazykové verze.

Úpravu samotné struktury, správu Moodlu a prípadné poradenství měla na starost Univerzitní knihovna Technické univerzity v Liberci a jednotliví partneři se tak mohli obracet s připomínkami a otázkami na pověřené osoby.

Modul byl přeložen během léta 2018 a pro uživatele partnerských knihoven byl spuštěn na konci roku. 


\title{
POZNATKY ÚSPĚŠNÉ SPOLUPRÁCE
}

aneb Tipy pro úspěšnou realizaci přeshraničního projektu

\author{
Dana Petrýdesová \\ Blanka Konvalinková
}


Náš společný projekt Prostor učení - Knihovnická informační platforma je u konce. Na více než dvouleté cestě nás potkalo spoustu zajímavého a radostného, zároveň se však objevily problémy, které jsme na začátku projektu neočekávali. Řešení těchto problémů nebyla vždy procházka rưžovým sadem - musíme ale konstatovat, že nás velmi obohatilo o nové zkušenosti, které můžeme v budoucnu využít při realizaci dalších projektů.

Pokusili jsme se naši osobní zkušenost shrnout do několika bodů a chceme Vám tím nabídnout několik tipů, jak úspěšně realizovat přeshraniční projekt.

\section{Tip č. 1 - Pečlivé plánování}

Máte domluvené partnery a $v$ hlavě pripravený koncept projektu? Nezapomeňte pečlivě plánovat. Je nezbytné, aby projektové aktivity měly podporu od všech partnerů projektu. Ujistěte se, že všichni partneři správně pochopili všechny souvislosti, vědí, co všechno obnášejí a souhlasí s nimi. Ideální je vytvořit protokol ze setkání, se kterým se partneři seznámí a odsouhlasí jeho znění. Mǔžete se tím vyhnout nedorozuměním v průběhu projektu.

Dalším důležitým úkolem je sestavení rozpočtu. Určitě se Vám nepodaří sestavit presný rozpočet na celou dobu trvání projektu, ale pečlivou př́pravou a provedením průzkumu trhu se můžete k optimální variantě alespoň přiblǐzit. Čeští partneři musí prihlédnout také kurzovému riziku, kdy pohyb kurzu směrem dolů mưže způsobit větší finanční náročnost - partner pak na některé aktivity doplácí z vlastního rozpočtu. Pohyb kurzu nelze předvídat, ale je nutné s ním při prípravě rozpočtu počítat.

\section{Tip č. 2 - Publicita}

Povinná publicita je jedním z nejdůležitějších předpokladů úspěšného projektu, resp. bez správné publicity se neobejde žádný projektový výstup nebo informační materiál a je důležité ji nepodceňovat.

Veškeré projektové vístupy opatřujte správným logem Evropské unie ve správné velikosti a zároveň odkazem na Evropský fond pro regionální rozvoj, doporučováno je také použití loga Programu. Ideální je slovní uvedení, že projekt získal finanční podporu od Evropské unie. 
Činnosti v oblasti publicity prováději všichni projektoví partneři, kteří by měli označit:

- hmotné výstupy projektu (např. získané vybavení)

- tištěné materiály (např. publikace)

- veškeré propagační předměty

- webové stránky projektu

- prostory, kde probíhají školení nebo workshopy (např. umístění vlajek, log do prezentací, roll-upu apod.), zároveň musí být povinnou publicitou označeny prezenční listiny nebo pozvánky na akci

- prostory budovy, kde partner působí (např. roll-up)

$\checkmark$ průběhu projektu je vhodné o projektu informovat v médiích, at už tradičních (tisk, rozhlas, regionální TV) nebo elektronických (online periodika, sociální sítě), prípadně na dalších akcích, kterých se účastníte (např. konference, veletrhy apod.).

Potřebné informace pro správnou publicitu najdete v metodických materiálech na portálu Programu (www.sn-cz2020.eu), které doporučujeme pečlivě prostudovat a sledovat průběžně - čas od času se pokyny aktualizují.

\section{Tip č. 3 - Komunikace s kontrolním orgánem}

Z vlastní zkušenosti doporučujeme všem žadatelům o dotaci, aby včas a dostatečně komunikovali s kontrolním orgánem své země (CRR, SAB). Jejich pracovníci jsou ochotni poradit se všemi problémy, které vás mohou v prüběhu realizace projektu potkat. Je vhodnější problém nejdřive prokonzultovat s kontrolorem a společně se domluvit na postupu a řešení. Pokud se problém řeší až následně, mǔže se stát, že některé chyby už nebude možné zvrátit a budete finančně tratit či vracet část dotace.

\section{Tip č. 4 - Sdílení}

Sdílení informací a materiálů může všem partnerům projektu významně usnadnit „projektový život". Pro potřeby našeho projektu vznikla díky Vysokoškolské knihovně Zittau/Görlitz platforma, kam byly ukládány všechny důležité materiály a informace (fotodokumentace, prezenční listiny, organizační informace apod.). Všichni partneři mají do platformy přístup a mohou elegantně používat všechny vložené materiály. Při vytváření zpráv o prüběhu projektu, tiskových zpráv nebo jiných informačních materiálů stačí pouze přihlásit se do platformy a všechny potřebné dokumenty si stáhnout. 


\section{Tip č. 5 - Společné řešení problémů}

Projekt, který má mít progresi, musí být řešen společně. Partneři by měli společně plánovat a realizovat aktivity a společně řešit problémy. Proto je určitě vhodné naplánovat přiměřený počet osobních setkání, workshopů nebo seminárù. V našem projektu jsme se velmi aktivně setkávali také virtuálně - pravidelně jednou za měsíc jsme diskutovali ve virtuální konferenční místnosti, kterou za tímto účelem žrizoval leadpartner Technická univerzita v Chemnitz. Někteří partneři pro tento účel využili technické vybavení, které pořídili z projektových finančních prostředků, někteří pouze vlastní technické prostředky. Samotná př́prava techniky a instalace není nijak složitá a vše lze zvládnout s minimálním úsilím. Vždy to byla skvělá přiležitost aktivně prodiskutovat problematické otázky a domluvit se na dalším vývoji projektu. Velmi se tím zvýšila komunikace mezi partnery a možnost aktuálně se navzájem informovat o novinkách, problémech a jejich řešení, aniž by bylo nutné navyšovat počet osobních setkání. Virtuální konference nám rozhodně ušetřila velké množství času.

\section{Tip č. 6 - Důraz na personál}

Velkým prínosem pro náš projekt bylo působení rodilých mluvčích v projektových týmech u německých partnerů, resp. pracovníků, kteří hovořili česky. Velmi to usnadnilo a zrychlilo komunikaci mezi partnery. Sebekriticky musíme přiznat, že čeští partneři tomuto faktoru nevěnovali při plánování personálu přiliš velkou pozornost, ocenili ale tuto skutečnost při každé příležitosti, at' už při mailové komunikaci, ve virtuálním prostředí nebo i při osobních setkáních. Děkujeme za tento dobrý nápad a určitě to je zajímavý tip pro jakýkoliv další projekt.

Samož̌ejmostí by mělo být, že pracovníci, kteří se projektu účastní, by měli být zodpovědní, pružní, se schopností řešit problémy operativně. Přínosná je také alespoň orientační znalost prostředí přeshraničních projektů a principů jejich administrativy. Ale asi není nezbytnou podmínkou, vše se dá rychle naučit.

\section{Tip č. 7 - Nebát se}

Jedno české přísloví říká: „Kdo se bojí, nesmí do lesa”. Toto přísloví také charakterizuje náš poslední tip pro úspěšný projekt. Realizace přeshraničních projektů může přinést mnohá úskalí, ale společnou prací partnerů se dá vše 
vyřešit. Prínos, který uskutečněním projektu je možné získat, preváží nad př́padnými negativními zkušenostmi. Mnohdy se to, co se zdá neuskutečnitelné nebo organizačně velmi náročné, mǔže změnit v zajímavou aktivitu, která může sloužit dlouhodobě po skončení projektu. Přeshraniční projekty jsou nejen možností s partnery spolupracovat na odborných tématech, ale vzájemně se obohacovat o osobní zkušenosti a rovněž poznat to, co je v obou zemích rozdílné a může být inspirativní. Kromě toho se časté pracovní setkávání partnerů může změnit i v hezké vzájemné prátelství, což prǐnáší další nezanedbatelný prínos projektu. Držíme Vám palce. 



\section{OTÁZEK PRO 5 PARTNERŮ}




\section{Angela Malz}

\section{Univerzitní knihovna Technické univerzity Chemnitz}

\section{Proč jste se rozhodla pro účast na tomto projektu?}

Česko-saská spolupráce má na Technické univerzitě Chemnitz dlouhou tradici a je úzce spojena s bohužel př́liž brzy zesnulým panem prof. Dr. Peterem Juzczekem, který tuto spolupráci aktivně inicioval.

V roce 2003 odstaroval první projekt, Česko-saské vysokoškolské centrum (ČSVC). V rámci tohoto projektu získala Univerzitní knihovna Chemnitz prostředky na výstavbu Česko-saské odborné knihovny (ČSOK). Knihovní fond sloužil k podpoře přeshraniční vědecké spolupráce mezi Českou republikou a Saskem.

Univerzitní knihovna Chemnitz pořídila knihy v německém a českém jazyce $s$ tématikou česko-německé spolupráce. Postupně je pak rožšriila o odborné knihy z oblasti ekonomie, dějin Saska a Čech nebo rož̌îrení Evropské unie na východ. Do fondu pribyla také beletristická díla, jazykové a turistické průvodce, mapy, DVD nebo audioknihy.

V roce 2006 projekt ČSVC skončil. V následném projektu Česko-saském vysokoškolském kolegiu získala odborná knihovna opět k dispozici další prostředky.

V létě 2009 odstartoval projekt Česko-saská vysokoškolská iniciativa, kdy poprvé hrály knihovny zúčastněných univerzit v projektové žádosti významnější roli. Vedle rozšrir̃ení Česko-saské odborné knihovny byl kladen důraz na přeshraniční výpůjční službu a posílení spolupráce mezi knihovnami.

Partnery Univerzitní knihovny Chemnitz tehdy byla Knihovna Univerzity Jana Evangelisty Purkyně (UJEP) v Ústí nad Labem a Univerzitní knihovna Západočeské univerzity v Plzni.

Na konci projektu již bylo zřejmé, že knihovny chtějí spolupracovat i nadále a zapojit do česko-saského príhraniči i další knihovny.

Od 01.12.2013 do 30.11.2014 byl realizován projekt Česko-saská sit' knihoven - informace bez hranic. Projektovými partnery byla Univerzitní knihovna Technické univerzity Chemnitz, Vysokoškolská knihovna Zittau/Görlitz, Univerzitní 
knihovna Západočeské univerzity v Plzni a Krajská vědecká knihovna v Liberci. Cílem projektu bylo vytvoření udržitelné a efektivní sítě knihoven mezi Českou republikou a Saskem.

Pozitivní zkušenosti a osobní kontakty navázané v tomto projektu vedly v roce 2015 k rozhodnutí podat žádost o nový projekt Prostor učení - Knihovnická informační platforma v rámci Programu na podporu přeshraniční spolupráce mezi Českou republikou a Svobodným státem Sasko 2014-2020.

\section{Jak Vaše knihovna z účasti na projektu profitovala?}

Profitovali jsme materiálně. Zde bych uvedla jen dva př́klady: elektronické knihy od Taylor \& Francis bychom bez projektových prostředků nemohli využívat. Také whiteboard bychom z vlastního rozpočtu financovat nemohli.

Cennější než materiální hodnoty byly ty lidské. Poznali jsme vysoce kvalifikované knihovníky a knihovnice, které nás velmi srdečně přijali. Dozvěděli jsme se hodně o českém knihovnictví a zjistili jsme, že stojíme před stejnými výzvami digitální společnosti. Tyto výzvy Ize zvládnout jen přeshraničně.

\section{Jaké projektové výsledky budete v knihovně využívat i po skončení projektu?}

Neexistuje žádný výsledek projektu, který by nebyl využitelný i po jeho skončení. Můžeme dál využívat elektronické zdroje, máme aktuální moderní techniku, dvojjazyčné katalogy, internetové stránky a i nadále využitelný e-learningový model k zprostředkování informační kompetence. Vytvořená informační platforma bude i nadále udržována a tím i vzájemné propojení knihoven.

\section{Jaké zkušenosti si z projektu odnesete? Chtěla byste něco udělat lépe nebo vylepšit?}

Jak jsem již zmínila, uvědomili jsme si, že všichni stojíme před stejnými výzvami digitální společnosti a mezinárodní zkušenosti nám v každodenní práci velmi pomáhají. Tyto zkušenosti nemusí pocházet pouze ze západního světa. I u našich sousedů na východě od Německa Ize najít mnoho chytrých nápadů. 


\section{Vnímáte potenciál pro rozšíření o další projekt?}

V každém případě. Nejprve však potřebujeme malou přestávku. V současné době nás totiž velmi zaměstnává plánované přestěhování do nové budovy $\checkmark$ roce 2019. V nové budově budeme mít určitě nové nápady na další projekt. Dokážu si představit např́klad téma open science, které v sobě skrývá velký potenciál. Ale také přeshraniční využívání elektronických médií není ještě zdaleka vyčerpáno. 


\section{Falk Maiwald / Ralf Schwarzbach} Vysokoškolská knihovna Vysoké školy Zittau/Görlitz

\section{Proč jste se rozhodla pro účast na tomto projektu?}

Pro účast na projektu jsme měli mnoho motivů a cílů. Díky předurčené poloze v Trojzemí a v rámci odpovídající strategie internacionalizace naší vysoké školy je dosažení vize propojeného vědeckého prostředí v Euroregionu Nisa i posláním Vysokoškolské knihovny Zittau/Görlitz (VŠK). V předcházejícím projektu jsme dosáhli již velmi dobrých výsledků a nasbírali cenné zkušenosti v přeshraniční spolupráci s ostatními vědeckými knihovnami. Odvodili jsme přitom důležité impulzy $\mathrm{k}$ další modernizaci a internacionalizaci knihoven v zájmu jejich uživatelů.

V novém projektu jsme spatřili šanci pořídit společně s našimi partnery v rámci konsorcia nová elektronická média a přeshraničně je využívat. S ohledem na změněné nároky prostoru učení na knihovny nás lákala také možnost zásadní modernizace mediálně-technické infrastruktury. A samožrejmě jsme byli zvědaví na transfer vědomostí z ostatních univerzitních knihoven.

Jako další motiv pro účast na projektu bych zmínil zlepšení image VŠK jak uvnitř tak navenek. V práci na projektu a přezkoumání nových témat a konceptů pro strategický rozvoj knihovny jsme uviděli šanci motivovat náš knihovní tým a mít tak efektivně vliv na vysokou školu. Jelikož jsou výzkumné projekty na odborných vysokých školách spiše vzácné, chtěli jsme díky projektu přitáhnout také pozornost regionu i celého Saska.

Od začátku pro nás bylo důležité sledovat v projektu jasné cíle a projekt zásadně spoluutvářet tak, abychom dosáhli konkrétních pozitivních výsledků pro nás i všechny projektové partnery.

\section{Jak Vaše knihovna z účasti na projektu profitovala?}

Hlavním přínosem pro naší knihovnu bylo samož̌ejmě konsorciální pořízení elektronických knih od Taylor \& Francis, přístup k databázím elektronických časopisů od Taylor \& Francis a Vogue, aktivní přeshraniční výpůjční služba nebo nakoupení mediální techniky. Dále pak zprostředkované resp. vyvinuté 
know how o médiích, knihovních fondech i databázích ostatních projektových partnerů včetně využivání mediální techniky pro webové konference a virtuální skupinovou práci. Také jsme získali důležité znalosti v oblasti publikování v Open Access, díky čemuž jsme zviditelnili toto téma v interní vysokoškolské diskuzi a mohli tím podpořit rozvoj strategie Open Access na naší vysoké škole.

Dalším důležitým prínosem bylo sblížení se všemi projektovými partnery a získání nových znalostí a zkušeností. Ted' se známe (ještě) lépe, máme lepší představu, jak fungují nejrůznější knihovnické záležitosti a situace v Čechách a v Německu a známe i důvody, proč tomu tak je. V oblasti informačního vzdělávání jsme mohli využívat zkušenosti a dokonce i specifické obsahy leadpartnera.

Neměli bychom zapomenout ani na kulturně-kulinářské toulky po Plzni a Liberci, při kterých se díky vzájemné (inter)kulturní inspiraci sblízili všichni projektoví parteři. Jako organizace profitovala naše knihovna z kolektivních zkušeností: zaměstnanci knihovny mohli strávit společný čas s ostatními kolegy a vyměnit si své znalosti. Každý pro sebe získal také své vlastní zkušenosti a nové podněty pro svou práci i pro sebe.

V projektu jsme mimoto představili výsledky naší spolupráce i veřejným regionálním knihovnám, které mohly vidět, co jsme společně s projektovými partnery vybudovali. Projekt získal uznání i ve Spolku saských vysokoškolských knihoven a byl zde vnímán jako průkopnický. Tento cíl, zlepšit pomocí projektu naši image na venek i uvniťr, považujeme za splněný.

\section{Jaké projektové výsledky budete v knihovně využívat i po skončení projektu?}

Po skončení projektu budeme samozřejmě i nadále využívat pořízená média; mimoto usilujeme o pokračování přeshraniční meziknihovní výpůjční služby. Dále budeme cíleně rozšiřovat využívání mediální techniky. Díky informační platformě se mohou uživatelé ostatních knihoven informovat o knihovním fondu a službách. Jako podpora budou sloužit odpovídající reklamní opatření a ústní propagace.

Zkušenosti v plánování a organizaci - i přeshraničních - konferenčních porad jak klasických tak virtuálních, můžeme využívat sami nebo dále zprostředkovávat ostatním aktérům naší vysoké školy. 
Kooperace s projektovými partnery zůstane v každém prípadě zachována. I v budoucnu spolu budeme komunikovat, scházet a radit se.

Euroregion Nisa bude jako vědecké centrum posilovat a i nadále bude docházet ke kulturní výměně, kterou již delší dobu udržujeme nad rámec projektu, především s Krajskou vědeckou knihovnou v Liberci.

\section{Jaké zkušenosti si z projektu odnesete? Chtěla byste něco udělat lépe nebo vylepšit?}

V projektu jsme nasbírali zkušenosti o projektových partnerech, jejich způsobu práce, podmínkách, které jsou u každého jiné (především rozdíly mezi Českou republikou a Německem). Také jsme se přesvědčili, že dokážeme vést konference na vysoké úrovni, navíc za velmi dobré organizace a většinou hladkého průběhu. Jsme nyní schopni uchopit nová témata realizovaná v partnerských knihovnách, př́padně je sami etablovat - nová technika, propojení díky meziknihovní výpuijční službě - a využít je i v rámci spolku. Podle vedení projektu VŠK Zittau/Görlitz bylo přínosné a pro dosažení cílů projektu rozhodující získat jako centrální projektovou pracovnici pro plánování, kontrolu, koordinaci a realizaci českou rodilou mluvčí s velmi dobrými znalostmi německého jazyka.

Díky pravidelně pořádaným webovým konferencím jsme zjistili, že virtuální konference mohou ušetřit mnoho času a jsou dobrou základnou pro koordinaci aktivit. Samožrejmě jsou osobní setkání a osobní kontakty velmi důležité. Pomocí obou zpưsobů se nám podařlo zajistit pokrok projektu. V rámci webových konferencí jsme vedli celou řadu prínosných diskuzí a nemuseli jsme se spoléhat pouze na občasná setkání (náročná na cestování) prímo na místě nebo ping-pongovou emailovou komunikaci.

Jako efektivní se osvědčilo rozdělení zodpovědností za jednotlivé milníky mezi konkrétní projektové partnery, což zajistilo splnění výsledků projektu.

\section{Vnímáte potenciál pro rozšíření o další projekt?}

Pokračování myšlenky Open Access a další optimalizace procesu informačního vzdělávání jistě stojí za další zpracování projektovými partnery. 


\section{Miloslava Faitová / Magda Šrajbová}

Univerzitní knihovna Západočeské univerzity v Plzni

\section{Proč jste se rozhodla pro účast na tomto projektu?}

Zásadní vliv na rozhodnutí účastnit se řešeného projektu měla moje všestranně dobrá zkušenost s realizací předchozích projektů podpořených z Evropského fondu pro regionální rozvoj v programu Cíl 3 žadatelským subjektem, konkrétně projektů Česko-saská vysokoškolská iniciativa (2009-2012) a Česko-saská sit' knihoven - Informace bez hranic (2013-2014).

Prostřednictvím nového projektu jsem chtěla navázat na úspěšnou a oboustranně velmi dobře hodnocenou spolupráci s partnerskými knihovnami z předchozích projektů, tedy s knihovnami v príhraničních oblastech Česka a Saska. Považovala jsme za nosné a efektivní pokračovat ve výměně zkušeností v osvědčených formátech workshopů, seminářủ a stáží v dlouhodobější perspektivě a dále tímto způsobem rozvíjet aktivity jednotlivých knihoven.

Za důležitou pro posílení služeb Univerzitní knihovny ZČU v Plzni jsem pokládala příležitost k rozšir̃ení fondu elektronických informačních zdrojů, jejichž pořízení bylo možné financovat z prostředků projektu.

K rozhodnutí účastnit se projektu přispěla také má předchozí znalost a zkušenost s podmínkami pro realizaci a administraci projektu daného typu.

\section{Jak Vaše knihovna z účasti na projektu profitovala?}

Důležitým přínosem projektu bylo především konsorciální pořízení elektronických informačních zdrojů a titulů e-knih z prestižního vydavatelství Taylor \& Francis. Univerzitní knihovna ZČU díky realizaci projektu získala přístup ke dvěma kolekcím databáze vědeckých časopisů (Social Science \& Humanities Library, Science \& Technology Library) v letech 2017-2018 a př́istup k 5 tisícům e-knih po dobu 1,5 roku s možností trvalého nákupu nejvyužívanějších titulů.

Byla rozšîrena příruční knihovna německé literatury, tzv. semestrální aparát, v Pedagogické knihovně ZČU, který slouží studentům germanistům jako zdroj pro práci v seminární výuce a ke státním zkouškám. 
Bylo nakoupeno potřebné technické vybavení pro knihovnu - notebooky a dataprojektor.

Studentům a akademickým pracovníkům ZČU v Plzni byla nabídnuta a zprostředkována jedinečná možnost mezinárodní meziknihovní výpůjčky knih zdarma z německých knihoven Technické univerzity v Chemnitz a Vysoké školy Zittau/Görlitz.

V rámci workshopů a seminár̊u byly plodně sdíleny a diskutovány znalosti a zkušenosti českých a německých partnerských knihoven, a to zejména v oblasti informačních kompetencí, technického vybavení knihoven, elektronických informačních zdrojü, podpory Open Access k publikovaným výsledkưm vědy a výzkumu a i dalších aktuálních témat.

Při pobytových stážích se zaměstnanci knihovny podrobněji seznámili s činností spolupracujících knihoven a vyměnili si zkušeností v intenzivním kontaktu. Měli tak možnost proniknout do chodu partnerských institucí hlouběji.

$\checkmark$ neposlední řadě umožnila realizace projektu uspořádat seminár pro doktorandy univerzit k aktuální problematice Open Acces.

\section{Jaké projektové výsledky budete v knihovně využívat i po skončení projektu?}

Výsledky trvalé hodnoty jsou:

- prístup k fondu e-knih vydavatelství Taylor\&Francis

- pokračování mezinárodní meziknihovní výpůjční služby s Univerzitní knihovnou Technické univerzity Chemnitz a Vysokoškolskou knihovnou VŠ Zittau/Görlitz

- využití technického vybavení pro činnosti v knihovně

- navázané a rozvinuté kontakty z partnerskými knihovnami

\section{Jaké zkušenosti si z projektu odnesete? Chtěla byste něco udělat lépe nebo vylepšit?}

Zkušenosti s realizací projektu jsou pozitivní a motivující pro další práci. Především bych chtěla vyzdvihnout výbornou, tj. prátelskou, otevřenou, efektivní a operativní spolupráci s partnerskými knihovnami a zdůraznit skutečné prohloubení vzájemné spolupráce. 
Podařilo se nám zjistit, jak to v jednotlivých knihovnách funguje, navzájem si představit řešení různých aktuálních výzev, sdílet příklady dobré praxe a nabyté znalosti a zkušenosti promítnout do kvality námi poskytovaných služeb.

Po dobré zkušenost s videokonferencemi a webináři bychom v budoucnu tyto aktivity dále rozvíjeli a tudíž zvažovali pořízení kvalitního vybavení pro jejich realizaci.

I při opakované zkušenosti byla administrace projektu poměrně složitá, i když mohu konstatovat dobrou komunikaci s Centrem pro regionální rozvoj.

\section{Vnímáte potenciál pro rozšiření o další projekt?}

0 dalším projektu v Programu na podporu přeshraniční spolupráce mezi Českou republikou a Svobodným státem Sasko v dotačním období 2014-2020 neuvažujeme a ani už by ho nebylo možné realizovat.

Pokud bude $v$ dalších letech vyhlášen nový program, z kterého by aktivity projektu mohly být podporovány, budeme zvažovat, zda se zapojit.

Oblast knihovnictví musí stačit trendům ve vzdělanosti a informovanosti společnosti, nadále bude potřeba zajištovat pro uživatele knihoven odpovídající knihovní fond, technické vybavení, což vyžaduje nemalé finanční náklady. Zajištění odpovídajících služeb klade na knihovníky nové požadavky, které je dobré ověřovat při výměně zkušeností s českými i zahraničními knihovníky. 


\section{Blanka Konvalinková / Dana Petrýdesová \\ Krajská vědecká knihovna v Liberci}

\section{Proč jste se rozhodla pro účast na tomto projektu?}

Krajská vědecká knihovna v Liberci byla součástí předchozího projektu, $s$ účastí v něm jsme byli spokojeni, logicky jsme se tedy zapojili do projektu nynějšího. Očekávali jsme další prohloubení a výměnu zkušeností a možnost získání elektronických zdrojů.

\section{Jak Vaše knihovna z účasti na projektu profitovala?}

V projektu jsme si naplánovali aktivity, které bylo třeba naplnit. Ceníme si získání elektronické databáze Vogue a možnosti zařídit náš přednáškový sál moderní technikou umožňující nahrávání přednášek a jejich přímý přenos na YouTube. Vždy jsme preferovali setkávání se všemi partnery projektu - komunikace s nimi byla vždy užitečná z profesního i lidského hlediska.

\section{Jaké projektové výsledky budete v knihovně využívat i po skončení projektu?}

Určitě již výše uvedené - databázi Vogue a zařízení v sále.

\section{Jaké zkušenosti si z projektu odnesete? Chtěla byste něco udělat lépe nebo vylepšit?}

Potěšilo mě velké nasazení zejména u lead partnera a v Hochschule Zittau/ Görlitz. Oba partneři zapojili do projektu česky mluvící kolegyně a to průběh projektu hodně zjednodušilo. Zkušenosti projektového manažera si spíš odnáší kolegyně Petrýdesová, ona pracovala s konkrétními aktivitami a čísly. Ocenila jsem možnost elektronické komunikace - ušetřila nám hodně času. Možná by projektu prospělo, kdyby byl o něco kratší a intenzivnější - pocituji, že přece jenom je naše knihovna charakterem odlišná od univerzitních knihoven - a některé podněty a problémy VŠ knihoven se naší knihovny netýkají - a naopak. Ale na druhou stranu - jako sonda do života univerzitních knihoven mi projekt přišel zajímavý. 


\section{Vnímáte potenciál pro rozšíření o další projekt?}

Jak už jsem uvedla, naše knihovna se typově odlišuje od ostatních knihoven v projektu. Nemůže tedy být ostatním partnerům rovnocenná. Pokud bychom se měli do pokračování projektu zapojit, účast naší knihovny bych považovala za přínosnou spíše v kontextu širší přeshraniční spolupráce $s$ dopadem na nejen akademickou veřejnost příhraniční oblasti Česko - Německo. Potenciál vidím v ještě užším propojení zúčastněných institucí a studentů škol. 


\section{Jitka Vencláková}

\section{Univerzitní knihovna Technické univerzity v Liberci}

\section{Proč jste se rozhodla pro účast na tomto projektu?}

Byla to pro nás dobrá př́ležitost. Z povzdálí jsem pozorovala projekt partnerů v minulém období, kterého se naše univerzita neúčastnila. Při př́pravě současného projektu jsem neváhala zapojit i naši knihovnu. Zajímají mě zkušenosti z ostatních univerzitních knihoven z Čech i zahraničí a spolupráce s nimi je určitě prínosem.

\section{Jak Vaše knihovna z účasti na projektu profitovala?}

Zkušenosti. Získali jsme spoustu cenných zkušeností nejen týkajících se odborných knihovnických procesů (např. meziknihovní výpůjční služby, institucionální repozitáře), ale i zkušeností s pořádáním workshopů a podobných akcí s účastí zahraničních kolegů. Pro mne je to první mezinárodní projekt, kterého se účastním a vyzkoušela jsem si na něm své manažerské schopnosti. Pro naše uživatele je velkým přínosem nové vybavení prostoru učení, které je jim volně k dispozici (sluchátka, interaktivní panel a př́islušenství k online konferencím).

\section{Jaké projektové výsledky budete v knihovně využívat i po skončení projektu?}

Vybavení. Po skončení projektu budou mít uživatelé nadále možnost využívat nové vybavení prostoru učení. Pokračovat bude předplatné kolekcí Science \& Technology Library a Business, Management \& Technology databáze Taylor \& Francis. Trvale dostupné budou i vybrané tituly e-knih z databáze Taylor \& Francis. V našem repozitáři bude přehledně dostupná v patřičném režimu zpřístupnění publikační činnost akademických pracovníků Technické univerzity v Liberci. K dispozici bude nová platforma informačních komptencí, která odpoví na otázky novým, stávajícím i potencionálním uživatelưm. Díky propagačním předmětům budeme moci nadále upozorňovat na výhody a smysl zapojení se do projektů v rámci Programu na podporu přeshraniční 
spolupráce mezi Českou republikou a Svobodným státem Sasko 2014-2020 a jemu podobných.

\section{Jaké zkušenosti si z projektu odnesete? Chtěla byste něco udělat lépe nebo vylepšit?}

Schopnosti. Já osobně jsem se ujistila o tom, že takový projekt společně s kolegy zvládnu naplánovat, realizovat i dokladovat. Pro př́ště se určitě budu více věnovat plánování. Ve chvíli, kdy je vše přesně naplánovaná činnost, personální pokrytí i finance, je realizace projektu mnohem snazší.

\section{Vnímáte potenciál pro rozšíření o další projekt?}

Ano, potenciál určitě vnímám. Určitě by bylo zajímavé např. prohledávání informačních zdrojů všech partnerů v rámci jednotlivých katalogů. Společné akce a vzájemná propagace by nesměla chybět. 